Bharat Goel

Arijit Sen

\title{
Appropriative Conflicts and the Evolution of Property Rights
}

Max Planck Institute for Tax Law and Public Finance Working Paper 2019 - 06

May 2019

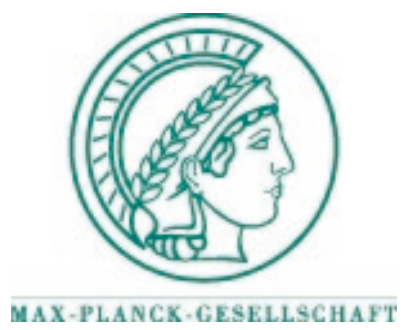

Max Planck Institute for Tax Law and Public Finance

Department of Business and Tax Law Department of Public Economics 
Working papers of the Max Planck Institute for Tax Law and Public Finance Research Paper Series serve to disseminate the research results of work in progress prior to publication to encourage the exchange of ideas and academic debate. Inclusion of a paper in the Research Paper Series does not constitute publication and should not limit publication in any other venue. The preprints published by the Max Planck Institute for Tax Law and Public Finance represent the views of the respective author(s) and not of the Institute as a whole. Copyright remains with the author(s).

Max Planck Institute for Tax Law and Public Finance

Marstallplatz 1

D-80539 Munich

Tel: $\quad+498924246-0$

Fax: $\quad+498924246-501$

E-mail: ssrn@tax.mpg.de

http://www.tax.mpg.de 


\title{
Appropriative Conflicts and the Evolution of Property Rights
}

\author{
Bharat Goel
}

Arijit Sen

Weak property rights over 'own fruits of labor' can provoke appropriative conflicts. However, the recognition of consequent resource-misallocation and value-destruction can encourage collective action towards improving property rights enforcement. We model two (pre-modern) communities populated by a sequence of myopic generations, initially located in a weak property-rights regime with unequal resources. In every generation, the communities costlessly strengthen, or weaken, inherited property rights by mutual consent, and then allocate inherited resources (whose amounts depend on past production and consumption) towards value creation and capture. The co-evolution of the property rights regime and resource inequality does not guarantee the emergence of perfect property rights enforcement in the long-run, essentially due to perverse incentives of the resource-poorer community. In fact, when initial resource-inequality is significant and/or the level of past productive investment (rather than the level of past consumption) is the dominant driver of a community's resource-growth, the poorer community drags the richer community into perpetual anarchy. When the communities are on an equilibrium path of devolution to anarchy, a policy intervention that exogenously and permanently imposes perfect property rights on them can lead to higher aggregate resource growth and higher welfare in each community in the long run, but can also cause an initial set of generations in the poorer community to be worse off compared to the laissez faire equilibrium path.

Keywords: Anarchy, appropriation, conflicts, contests, property rights.

JEL classification: C72, C78, D23, D74.

\section{Introduction}

"The efforts of men are utilized in two different ways: they are directed to the production or transformation of economic goods, or else to the appropriation of goods produced by others."

Vilfredo Pareto [quoted in Hirshleifer (1994)]

"What is the structure of property rights in a society at a point of time? ... How has this property right structure come into being?"

Alchian and Demsetz (1973)

It is by now well established that economic outcomes are worse in societies with poor property rights, largely due to the fact that when property rights are imperfect (either incompletely specified or imprecisely enforced) economic agents are more inclined to appropriate rival value rather than to

Authors' Coordinates: Goel: Max Planck Institute for Tax Law and Public Finance, Marstallplatz 1, 80539

Munich, GERMANY; bharat.goel@tax.mpg.de; Sen: IIM Calcutta, Kolkata 700104, INDIA;

arijitsen@iimcal.ac.in 
Acknowledgements: The authors are grateful for helpful comments from conference participants at the 2016 UEA Contest Conference in Norwich and at the $5^{\text {th }}$ Game Theory World Congress at Maastricht. create own value. ${ }^{1}$ At the same time, it is reasonable to suppose that the recognition of resourcediversion towards value-capture and of value-destruction caused by appropriative conflicts will encourage economic agents (especially non-atomistic ones) to work collectively towards improving the property rights regime and its enforcement. It is in this sense that the two issues referred to in the above quotes are intrinsically interconnected.

This paper explores the logic and the nature of this interconnection. We do so in the context of interactions between two long-lived (pre-modern) communities that have to make, in every generation, a collective choice regarding property rights enforcement and individual choices over value creation and appropriation. Our analysis focuses on $(a)$ the nature of inefficiencies that arise from strategic choices over value creation $v s$. capture, $(b)$ the intertemporal evolution of economic resources and welfare that the choices generate, and $(c)$ the consequent short-term incentives that the communities have in every generation to take collective action to strengthen (or weaken) the property rights regime that they inherit. We study the interaction effects between an evolving property rights regime that is 'nudged' (possibly incrementally) over time by generations of myopic community residents, the within-generation choices between value creation and appropriation in each community, and their implications for resource and welfare growth.

Our research relates to a large literature that addresses the following issues: $(a)$ appropriative conflicts in 'state of nature models' of value creation and capture, and $(b)$ endogenous choice of the structure and the level of enforcement of property rights in society. Skaperdas (1992), Grossman and Kim(1995), Hirshleifer (1995), and Neary (1997) are the leading contributions on the first issue. Important initial research on the second issue - within the context of state-of-nature models of appropriative conflicts - has been carried out by Genicot and Skaperdas (2002), Kumar (2008), and McBride, Milante, and Skaperdas (2011). We extend the inquiry on the relationship between appropriative conflicts and property rights enforcement by building on these seminal papers.

\subsection{Overview of the Model}

We study intertemporal strategic interactions between two (pre-modern) communities populated by generations of residents. In each generation (time period), the contemporary residents of the communities first decide to bring about (incremental) changes in their inherited property rights regime via collective action. Each community then decides on how to allocate its resource endowment between productive use (creating butter) and combative use (assembling guns), given the possibility of a subsequent appropriative conflict arising out of each community's attempt to

\footnotetext{
${ }^{1}$ Besley and Ghatak (2010) survey the large economics literature which studies the detrimental effects of weak property rights on economic outcomes.
} 
capture rival value. After the guns and butter outputs of each community are realized, and before any value-destroying appropriative conflict can occur, the communities have the opportunity to negotiate a peace agreement, in which one community 'buys peace' by offering an appropriate amount of 'butter bribe' to the other. We refer to this game of value creation and capture played in every generation between the two communities as the 'guns-and-butter game'. 2, 3

The collectively-chosen property rights regime $\pi \in[0,1]$ in a generation is its common 'security parameter' reflecting the extent of property-rights enforcement in the generation: $\pi$ represents the secured proportion of 'own fruits of labor' (fraction of own created butter that is protected from rival appropriation). Choice of $\pi$ in a generation leads to the next generation inheriting the right to choose its own rights regime from a 'rights space' that is a compact neighborhood of $\pi$ in $[0,1]$. The size of the rights space depends on the extent of 'institutional inertia' in the system. Given a generation's rights space, the contemporary residents of the two communities costlessly choose a rights regime from this space by mutual consent (before engaging in the guns-and-butter game).

Our (infinite) 'sequence-of-generations model' starts from a generation in which the inherited rights regime is 'imperfect $(\pi<1)$ ', and where the two communities are differentially-endowed with stocks of initial resources (aggregate inalienable human capital is a good example of such community resources). Then a pre-specified 'resource law-of-motion' determines the evolution of the resource endowment of each community across generations. We assume that resource growth (e.g., growth in human capital) is determined by past endowment level, the magnitude of past productive investments (which enhances skill acquisition), and past aggregate consumptions (which improves nutrition). As a result, the evolution of the property rights regime, and the generational resource endowments of the two communities as well as their production and consumption, become interconnected: the inherited rights regime and resource endowments induce a property rights choice in every generation, the established rights regime influences intra-generational production and consumption, and these outcomes determine the inheritances for the next generation.

The following three features of our model bear emphasis. First, we assume that every generation is myopic - each generation's goal in nudging the property rights regime and in making guns $v s$. butter

\footnotetext{
${ }^{2}$ In each generation, residents of the two communities are involved in two strategic interactions in their 'generational game': first in the collective choice of the property rights regime, and then in the guns-andbutter choices. In what follows, we refer to the first interaction as the 'rights negotiation game' and the second one as the 'guns-and-butter game'. Here we are obviously abusing the verbiage of game theory, but as our analysis will confirm, we correctly identify the unique SPNE outcome in the 'generational game'.

${ }^{3}$ Our guns-and-butter game is a generalization of many appropriative conflicts studied in the literature. Two particular features that co-exist in our game, and constitute a generalization over existing analyses, are the following: (i) if an appropriative conflict takes place, each community has to decide how to split its total guns output across offensive use (aimed at appropriating rival butter) and defensive use (aimed at protecting own butter), and (ii) the contestants can conclude credible peace negotiations in the shadow of future conflict.
} 
choices is to maximize its own final butter consumption. Our aim is to delineate how a sequence of within-generation (short-term) decisions about value creation vs. capture leads to a specific evolution trajectory for property rights enforcement (see Section 5.3 for more on this issue). Second, since property rights enforcement is a public good between the communities, we posit that the choice of the rights regime in every generation requires mutual consent (Section 2.2 clarifies how we model this requirement). Third, we assume that within their inherited property rights space, it is costless for the communities to collectively nudge the rights regime; this is because we want to demonstrate the possibility that the communities will (choose to) devolve to 'anarchy $(\pi=0)$ ' even when improving property rights only requires a collective will (and no physical resources).

It is worth clarifying that our focus is on the issue of economic agents deciding on the level of property rights enforcement in an environment where third-party enforcement is not available. Given that, we do not inquire as to how the idea of individual property rights (sense of ownership) evolves in humans and permeates through society (on that see Eswaran and Neary 2014). Further, even though we consider self-administration of rights enforcement by a collective of economic agents, we do assume that explicit property rights enforcement is necessary and possible (we do not study the issue of tacit (de facto) sustenance of implicit property rights under repeated interactions). ${ }^{4}$

\subsection{Summary of Results}

Our analysis contributes to the extant literature in two ways. Firstly, it delineates the equilibrium features of a generational guns-and-butter game - the game being more general than most that have been studied in the literature. Specifically, we identify conditions under which a contesting community will strictly prefer a weakening of the property rights (enforcement) regime in such a game. Secondly, our dynamic analysis generates an extensive set of results regarding the coevolution of endogenously chosen property rights regime, resource endowments, and the welfare of the communities. In this regard, we identify the critical role played by two factors: (i) inequality in initial resource endowments between the 'richer community' and the 'poorer community', 5 and

\footnotetext{
${ }^{4}$ Our focus on self-administration of beneficial societal rules by 'private collectives' is congruent with the discourse in Dixit (2004). Ensminger (1992) and Greif (2006) discuss historical instances in which private collectives (in Africa and in medieval Europe) enforced property rights. In contrast to our focus on de jure enforcement of property rights by private collectives, the possibility of de facto enforcement of property rights has been studied by Muthoo (2004) and Hafer (2006). The former paper considers an infinitelyrepeated game version of the state-of-nature model, and identifies conditions under which 'absence of appropriation' will be a subgame-perfect Nash equilibrium outcome. The latter paper studies tacit honoring of property rights in an economic environment where pairs of economic agents can engage in wars of attrition.

${ }^{5}$ Here, and subsequently, a community will be termed richer/poorer in any generation solely in terms of its relative resource endowment in that generation. Our formal analysis will establish that in our sequence-ofgenerations model, the communities' initial resource ranking will be preserved in all future generations; in equilibrium, there will be no intertemporal role reversal between the richer and the poorer community.
} 
(ii) the nature of the 'resource law-of-motion' that determines the evolution of resource inequality across generations, especially the relative dependence of resource growth on past productive investment and past aggregate consumption.

We start by discussing the generational guns-and-butter game. Consider any generation in which property rights are imperfect [ $\pi<1]$, and community 1 is strictly richer than community 2 ; let $R_{i}$ (resp., $W_{i}$ ) denote the resource endowment (resp., aggregate consumption) of community $i$, for $i=1,2$. Then a generational guns-and-butter equilibrium exhibits the following features:

- The poorer community will be more conflict-prone than the richer community in that the latter will have to pay a butter bribe to the former to buy peace (and dissuade it from initiating conflict).

- The Hirshleiferian 'paradox of power' will hold in that the consumption-resource ratio of the poorer community $\left(W_{2} / R_{2}\right)$ will be strictly greater than that of the richer community $\left(W_{1} / R_{1}\right){ }^{6}$

- There will be a divergence in induced preferences over property rights between the communities: the poorer community will have a weaker desire to strengthen property rights than the richer. In fact for sufficiently high resource inequality, there will be 'complete divergence in $\pi$-preference' between the communities: the richer community will prefer 'perfect property rights $[\pi=1]$ ' while the poorer community will prefer 'anarchy $[\pi=0]$ ' over any other rights regime.

In our sequence-of-generations model, it is the persistent divergence in $\pi$-preference (caused by forces that sustain resource inequality) - with the poorer community strictly preferring a weakening of the rights regime - that can prevent the infinite sequence of generations of residents in the two communities from ever attaining perfect property rights. In fact, the poorer community can drag the richer one into perpetual anarchy. The following long-run results hold in our dynamic model:

- Resource inequality between the communities and the endogenously chosen property rights coevolve over time (continuously impacting each other along the time path) in a way that the following is true: the equilibrium path devolves to perpetual anarchy (resp., attains perfect property rights) if and only if resource inequality permanently settles above a high threshold (resp., below a low threshold) in the long-run.

- When the law-of-motion that determines the evolution of resource inequality is 'well-behaved' in specific ways, then the following results hold: If past aggregate consumption has a substantial impact on a community's resource growth then resource inequality between the communities decreases over time and the institution of perfect property rights is eventually attained. In contrast, if initial resource inequality is significant and/ or past productive investment is the prime driver of resource growth then resource inequality increases over time and the property-

\footnotetext{
${ }^{6}$ In a conflict between asymmetric players, Hirshleifer (1991) considered the following phenomenon to constitute a 'paradox of power': the consumption inequality between the two contestants is less than their resource inequality: $\left(W_{2} / W_{1}\right)>\left(R_{2} / R_{1}\right)$. Of course, this condition is equivalent to our stated condition.
} 
rights regime devolves to perpetual anarchy. ${ }^{7}$

- When devolution to anarchy is the laissez faire equilibrium path, then a policy intervention that imposes perfect property rights in perpetuity will cause both communities to be better-off in the long-run, but not necessarily in the short-run; the poorer community might prefer the laissez faire path in the short-run even when each generation cares about its immediate progeny.

The rest of the paper is organized as follows. Section 2 contains the formal description of our sequence-of-generations model. We then present some illustrative examples in Section 3 that clarify important properties of generational equilibria as well as the dynamic equilibrium path. The static analysis of within-generation equilibrium behavior is contained in Section 4, where we verify the robustness of our results when some important model assumptions are relaxed. Section 5 presents our results for the equilibrium evolution of property rights, resources, and community welfare. We conclude in Section 6 by relating our results to the extant literature (such relations are also explained in many subsequent footnotes). An appendix contains the formal proofs of our results.

\section{The Sequence-of-Generations Model}

In this section, we elaborate on our sequence-of-generations model of intertemporal interactions between two (neighboring, pre-modern) communities by specifying the details of the following three components of our model:

(1) the guns-and-butter game played between the contemporaneous residents of the two communities in every generation, after the communities have received their resource endowments and have (collectively) chosen the property rights regime that will govern their interactions;

(2) the transition structure between consecutive generations that determines 'resource evolution' i.e., the dependence of the current resource endowment of a community on past history, and 'rightsregime evolution' - i.e., the determination of the property-rights space over which a generation can negotiate its rights regime as a function of history and of the extent of institutional inertia; and (3) the property-rights negotiation game between the communities in every generation, in which they collectively and costlessly choose their rights regime (after having received their resource endowments and before engaging in the guns-and-butter game).

\subsection{The Generational Guns - and - Butter Game}

The generational guns-and-butter game is a scenario of appropriative conflict in which the contemporary members of the two communities take as given the property rights regime (that they

\footnotetext{
${ }^{7}$ It will be clarified in Section 5 that the evolution of property rights will depend on the initial resource inequality, the extent of loss of value due to conflict, the across-generation resource law-of-motion, and the inherited rights space and the extent of institutional inertia in changing inherited property-rights.
} 
have collectively established) and their resource endowments, and then make strategic decisions regarding guns and butter production. In our analysis we ignore any within-community heterogeneity and consider each community to be a single decision-making unit.

The data of the guns-and-butter game in any generation are the inalienable resource endowments of communities 1 and 2: $R_{1}>0$ and $R_{2}>0$, and the chosen rights regime: $\pi \in[0,1]{ }^{8}$ It will be helpful to consider each community's resource endowment to be its embodied (and inalienable) human capital. As indicated in Section $1, \pi$ is the security parameter that measures the security of claims of each community regarding its own fruits of labor - for each community, $\pi \in[0,1]$ is the fraction of its productive output (butter) that is secure from appropriation by the rival community. ${ }^{9}$

Given $\left\{R_{1}, R_{2}\right\}$, we define $R \equiv R_{1}+R_{2}$ and $\rho \equiv\left[R_{2} / R\right]$; here $R$ is the total resource endowment of the communities, and $\rho$ parameterizes the extent of resource inequality between them (in what follows we will always study the case where $R_{1} \geq R_{2}$; thus, smaller $\rho$ will imply greater resource inequality). The generational guns-and-butter game is played in three stages:

STAGE 1: First, each community $i$ (for $i=1,2$ ) allocates its resources $R_{i}$ across two kinds of investments - a combative guns investment and a productive butter investment; e.g., viewing a community's resources to be its human capital, a part of this human capital is directed towards acquiring fighting skills (guns investment) and the remainder towards value creation (butter investment). ${ }^{10}$ If community $i$ invests $G_{i} \in\left[0, \mathrm{R}_{i}\right]$ in guns, its guns output (i.e., the size of its army) will be $G_{i}$ and its butter output will be $B_{i}=\left[R_{i}-G_{i}\right] .{ }^{11}$ In a rights regime $\pi \in[0,1], \pi$ fraction of

${ }^{8}$ The guns-and-butter game is played in every generation $t$, for $t \in\{1,2,3, \ldots\}$. In describing the structure of the game in a specific generation $t$, we are currently suppressing conditioning of all variables on $t$ to reduce notational burden. In every generation, the resource base $R_{i}$ of each community $i$ and the prevailing rights regime $\pi$ will depend on past histories and collective choices in specific ways.

${ }^{9}$ As indicated in Section $1, \pi \in[0,1]$ in effect represents the chosen level of enforcement of property rights in the generation. This security-parameter formulation of the property-rights regime is also present explicitly in Kumar (2008) and McBride et. al. (2011), and implicitly in Genicot and Skaperdas (2002). Note that an alternative, and analytically equivalent, interpretation of $\pi$ can be the following: A 'rights protecting force' is jointly (and costlessly) created by the two communities (by mutual consent) which locates itself along the boundary of the two communities (at the beginning of the guns-and-butter game) and repels every appropriative attack with probability $\pi \in[0,1]$; the bigger is the size of the force the larger is $\pi$.

${ }^{10}$ Note that in our model, 'guns' do not refer to durable weapons of war; rather, they refer to an amount of human capital that is trained in warfare within a generation (that perishes when the generation perishes). Further, we assume full utilization of resources by the communities - this is without any loss of generality as partial use of its resource endowment will be a strictly dominated strategy for each community.

${ }^{11}$ Our substantive assumptions regarding the production functions are: $(i)$ they are identical across the two communities, and (ii) each production function exhibits constant returns to scale. In Section 4.3, we establish the robustness of our results when there are limited non-constant returns to scale in guns production. For constant returns to scale in production, taking the production coefficients in the guns and 
community $i$ 's butter output $B_{i}$ will be secure against rival appropriation, while $\left[(1-\pi) B_{i}\right]$ amount of community $i$ 's butter output will be 'contestable' by community $j$. In the case where $\pi=1$, no butter is contestable, and the game concludes after resource allocations have been made, with each community $i$ retaining the butter output $B_{i}$ and earning final payoff which equals its aggregate butter consumption (i.e., its butter wealth) $W_{i}=B_{i}$. (Note that community resources are never contestable.)

STAGE 2: For any $\pi<1$, once the guns-and-butter vector $\left\{\left(G_{1}, B_{1}\right),\left(G_{2}, B_{2}\right)\right\}$ is realized, the two communities engage in peace negotiations. We do not specify an explicit bargaining protocol to model the peace negotiations; rather, we posit that the negotiation process culminates in the 'splitthe-surplus outcome' that is predicted by the Nash Bargaining Solution. In that outcome the communities reach the following kind of agreement: one community $i$ gives a butter bribe $b_{i j} \in\left[-B_{j}, B_{i}\right]$, which is a feasible transfer of some of its butter output, to the other community $j$, and both communities concurrently commit their armies solely to defense. ${ }^{12,13}$

As our subsequent analysis will clarify, our model has the following feature: after committing its army to defense as a part of a peace agreement, it will be sequentially optimal for each community to renege on its commitment (if it can) and attack the other's (post-bribe) butter output. In what follows, we focus on the case where each community's peace commitment is credible, i.e., once bilateral commitments are made, no community can unilaterally renege and use its army to attack its rival. ${ }^{14}$ However, we do indicate in Section 4.3 that our results survive in the "no-commitment scenario' where it is assumed that credible peace-treaties cannot be written.

STAGE 3: If no agreement emerges from the peace negotiations, then the game enters the following 'terminal conflict' phase: Given $\left\{\left(G_{1}, B_{1}\right),\left(G_{2}, B_{2}\right)\right\}$ and $\pi<1$, each community $i$ decides

butter production functions to be unity is only a simplifying assumption; our results will not change if the coefficients are different from unity (this is immediate from our posited payoff functions).

12 Peace negotiations occur if and only if $\pi<1$ and $\left[\left(G_{1} \times B_{2}\right)+\left(G_{2} \times B_{1}\right)\right]>0$. [If that is not the case, then the game concludes at the end of Stage 1 with each community consuming its own butter production, if any.] In the peace negotiations, outside options of the communities derive from their continuation terminal conflict payoffs (given that negotiation failure leads to terminal conflict). As the communities are risk-neutral (their payoffs are linear in total butter consumption), the split-the-surplus bargaining outcome coincides with other cooperative bargaining solutions like the Kalai-Smorodinsky Solution. The outcome can be implemented non-cooperatively by an alternating-offer bargaining game with a vanishing probability of breakdown.

${ }^{13}$ Viewing guns investment as generating a set of skilled warriors, a peace commitment involves garrisoning the warriors in a way that prevents them from launching an attack on the other community.

${ }^{14}$ In a one-generation model with imperfect property rights over productive resources, Anbarci, Skaperdas, and Syropoulos (2002) study a model in which two communities can engage in similar credible peace negotiations under the shadow of conflict. By considering strictly concave payoff functions for the two communities, the authors study the impact of alternative cooperative bargaining solutions on the ex ante guns and butter choices of the communities. 
on how to split its army $G_{i}$ into defensive forces $D_{i} \geq 0$ and attacking forces $A_{i} \geq 0$ such that $\left[D_{i}+A_{i}\right]$ $\leq G_{i}{ }^{15}$ If community $i$ sets $A_{i}>0$ and $D_{i}=0$ then it necessarily attacks its rival $j$ for the contested butter output $\left[(1-\pi) B_{j}\right]$, but does not defend its own contested butter output $\left[(1-\pi) B_{i}\right]$. Alternatively, if $i$ sets $A_{i}=0$ and $D_{i}>0$ then it only defends its own butter. If, however, community $i$ sets $A_{i}>0$ and $D_{i}>0$ then it concurrently defends its own butter and attacks rival butter. ${ }^{16}$ Whenever any community $j$ is attacked, its contested butter output $\left[(1-\pi) B_{j}\right]$ loses a fraction of its value and shrinks to $\sigma\left[(1-\pi) B_{j}\right]$; here, $\sigma$ parameterizes the 'post-conflict survival of value' (a smaller $\sigma$ implies a larger loss of value due to conflict) and we take $\sigma$ to be a fraction in $[1 / 2,1) .{ }^{17}$ Further, whenever community $i$ attacks community $j$ (with $A_{i}>0$ ), community $i$ appropriates the share $\alpha_{i}\left(A_{i}, D_{j}\right)$ of $\left[(1-\pi) \sigma B_{j}\right]$, leaving $\left[\pi+\left(1-\alpha_{i}().\right)(1-\pi) \sigma\right]$ fraction of $j$ 's output $B_{j}$ for community $j$. We assume that the 'contest success function' $\alpha_{i}($.) takes the simple 'lottery form':

$$
\alpha_{i}\left(A_{i}, D_{j}\right)=\left[A_{i} /\left(A_{i}+D_{j}\right)\right] \text { for any } A_{i}>0 \text { and } D_{j} \geq 0 .
$$

Thus, in the case where $A_{i}>0$ and $A_{j}=0$, only the butter output of community $j$ is attacked, and resultantly the final payoff to community $i$ is its aggregate butter consumption (its butter wealth) $W_{i}=\left[B_{i}+\alpha_{i}().(1-\pi) \sigma B_{j}\right]$ and the final payoff to community $j$ is $W_{j}=\left[\pi B_{j}+\left(1-\alpha_{i}().\right)(1-\pi) \sigma B_{j}\right]$. When $A_{1}>0$ and $A_{2}>0$, the butter outputs of both communities are attacked, and that generates the final payoff $W_{i}=\left[\pi B_{i}+\left(1-\alpha_{j}().\right)(1-\pi) \sigma B_{i}+\alpha_{i}().(1-\pi) \sigma B_{j}\right]$ for each community $i=1,2$.

Alternatively, if peace negotiations succeed and a peace agreement specifying a butter bribe $b_{i j} \in\left[-B_{j}, B_{i}\right]$ from $i$ to $j$ is reached then the bribe is paid and each community $k$ concurrently and irreversibly sets $A_{k}=0$. Then the game ends with community $i$ consuming butter output $\left[B_{i}-b_{i j}\right]$ and earning final payoff $W_{i}=\left[B_{i}-b_{i j}\right]$ and community $j$ consuming $\left[B_{j}+b_{i j}\right]$ and earning $W_{j}=\left[B_{j}+\right.$ $b_{i j} .^{18}$

${ }^{15}$ When $\pi<1$ and $\left[\left(G_{1} \times B_{2}\right)+\left(G_{2} \times B_{1}\right)\right]>0$, there can be no peace in our model in the absence of a credible peace agreement, since after the guns-and-butter vector is realized, no additional 'output/resource diversion' is necessary for a community with guns to start a war. This is in contrast to Bevia and Corchon (2010).

${ }^{16}$ When this is the case for both communities, the appropriative conflict is a 'two-battle conflict' - in one battle community 1 is the attacker and community 2 is the defender, while the roles are reversed in the other battle. However, in contrast to 'sequential multi-battle contests' studied by Konrad and Kovenock (2009), the two battles occur concurrently in our model.

${ }^{17}$ Garfinkel and Skaperdas (2000), McBride, Milante and Skaperdas (2011) and McBride and Skaperdas (2014) posit similar loss of value due to conflict. We consider it natural to posit that conflict causes some destruction, and so take $\sigma$ to lie between $1 / 2$ and 1 . Most of our results remain valid for $\sigma<1 / 2$ as well as for $\sigma=1$. One could more plausibly assume that $(1-\sigma)$ fraction of $B_{i}$ is destroyed in conflict if and only if both $A_{j}$ and $D_{i}$ are strictly positive; such a reformulation will not affect our results.

18 These payoff specifications reflect our assumption that each generation is myopic: in making its gunsand-butter decisions (and in its choice of property rights in the rights negotiation game), the aim of each 


\subsection{Generational Transition \& Rights Negotiation}

In our dynamic model, given the inherited resources and the rights negotiation space for generation $t$ $(t=1,2,3, \ldots)$, the vector $\left\{\pi(t), G_{1}(t), G_{2}(t), B_{1}(t), B_{2}(t), W_{1}(t), W_{2}(t)\right\}$ of the chosen rights-regime, guns production profile, and butter production and consumption profiles is its 'outcome vector'. Generation $t$ 's inheritance and outcome vectors give rise to the 'inheritance vector' $\left\{R_{1}(t+1)\right.$, $\left.R_{2}(t+1), \Pi(t+1)\right\}$ for the next generation $t+1$, where the inherited resource endowment $R_{i}(t+1)$ of each community $i$ is generated from the outcome vector in generation $t$ via a (common) resource law-of-motion $(R L M)$ function $F($.$) , and the inherited rights space \Pi(t+1)$ is a compact neighborhood of $\pi(t)$ in $[0,1]$ - determined by the extent of institutional inertia - from which the generation $t+1$ residents of the two communities select $\pi(t+1)$ in the rights negotiation game. We posit that at the end of every generation $t \geq 1$, the $R L M$ function $F($.) for each community $i$ has $\left\{R_{i}(t), B_{i}(t), W_{i}(t)\right\}$ as its arguments: $R_{i}(t+1) \equiv F\left\{R_{i}(t), B_{i}(t), W_{i}(t)\right\}$ for all $t \geq 1$ and $i=1,2$. We assume that $F($.$) is 'well-behaved': F\left(R_{i}, B_{i}, W_{i}\right)$ is strictly positive and continuously differentiable in its arguments, and it is strictly increasing in $R_{i}$ and non-decreasing in $B_{i}$ and $W_{i \cdot}{ }^{19,20}$

Regarding the inherited rights space $\Pi($.$) , we posit the existence of institutional inertia: there exists$ a vector $\{\varepsilon, \eta\} \in(0,1] \times(0,1]$ such that $\Pi(t+1) \equiv[\pi(t)-\varepsilon, \pi(t)+\eta] \cap[0,1]$, where $\pi(t)$ is the established rights regime in generation $t$. Here the idea is that while community residents can indeed alter inherited property rights to some degree, institutional inertia limits the extent of regime change that

generation in each community is to maximize its own final butter wealth. Further, butter is perishable, and no part of current butter wealth survives to the next generation.

${ }^{19}$ Note that we could equivalently consider the argument of the $F($.) function to be the generational outcome vector, given that it will always be the case that $R_{i}(t)=\left[B_{i}(t)+G_{i}(t)\right]$ for $i=1,2$ and for all $t$. We choose to specify the argument of $F\left(\right.$.) for each community $i$ to be $\left\{R_{i}(t), B_{i}(t), W_{i}(t)\right\}$ in order to conform to the following intuitive story. Viewing a community's resources to be its human capital, we think of resource evolution across generations as follows: The total human capital of a generation in a community is born at the time of birth of the generation and dies with the generation's death. Within a community, a generation's total human capital depends positively on the parents' total human capital, the productive skills acquired by the parents (by producing butter) and on the parents' nutrition (via their butter consumption). In our analysis in Section 5, we will also focus on a well-behaved RLM that is homogeneous and/or linear.

${ }^{20}$ Even though the focus of the two papers are quite different, Garfinkel and Skaperdas (2000) [GS] study a model which, though much simpler, has some similarities with our sequence-of-generations model. Firstly, [GS] consider a guns-and-butter game where, as in our model, the contestants can negotiate peace after the guns and butter outputs are realized; attack-defense splits of guns are not permitted in the [GS] model, and more importantly, the conflict occurs under anarchy (i.e., where $\pi$ is immutably fixed at zero) and resourceinequality between the two communities is small so that the conflict outcome is necessarily an interior equilibrium. Secondly, [GS] consider a dynamic extension which incorporates a simple resource law-ofmotion where resource-growth of a community depends only on past consumption/ utility. 
can be implemented in a single generation. ${ }^{21}$

As discussed earlier, we consider the property rights regime to be a public good between the two communities (it is a commonly shared institution between them) and assume that the communities make a collective choice from their inherited rights space by mutual consent; further, they do so in a costless manner and are motivated by their myopic goals. ${ }^{22}$ Specifically, we model the generational rights negotiation game as follows: In every generation $t$, given the (compact) inherited rights space $\Pi(t) \subseteq[0,1]$, the two communities - each of whose aim is to maximize own butter consumption announces the numbers $s_{1}(t) \in \Pi(t)$ and $s_{2}(t) \in \Pi(t)$ in a pre-specified sequence. Given the announcements $\left\{s_{1}(t), s_{2}(t)\right\}$, the property rights regime $\pi(t)=\min \left\{s_{1}(t), s_{2}(t)\right\}$ is established. $^{23}$ We end this section by summarizing our sequence-of-generations model starting from the first generation. The 'structural parameters' of our model are: $\sigma \in[1 / 2,1),\{\varepsilon, \eta\} \in(0,1] \times(0,1]$, and a wellbehaved $R L M$ function $F\left(\right.$.). The model's 'initial condition' is the inheritance vector $\left\{R_{1}(1), R_{2}(1)\right.$; $\Pi(1)\}$ of the first generation of residents in the two communities, where $R_{1}(1)>R_{2}(1)>0$ and $\Pi(1)$ is a compact subset of $[0,1]$. ${ }^{24}$ We let $R(1) \equiv\left[R_{1}(1)+R_{2}(1)\right]$ and $\rho(1) \equiv\left[R_{2}(1) / R(1)\right]$.

Given the structural parameters and the initial condition, the residents of the two communities in the first generation choose their rights regime and then play the guns-and-butter game, which results in guns production, butter production, and butter consumption outcomes in each community. These first-generation outcomes generate the resource endowments and the inherited rights space of the second generation community-members, who then make their decisions in the same sequence as

${ }^{21}$ The existence of a general 'status quo bias' can cause institutional inertia. While we do consider it realistic to assume some amount of such inertia, most of our results hold for all $\{\varepsilon, \eta\} \in(0,1] \times(0,1]$, i.e., even when there is no such inertia. Note that any $\eta>0$ (resp., any $\varepsilon>0$ ) will permit perfect property rights $[\pi=1]$ (resp., anarchy $[\pi=0]$ ) to be eventually attained.

${ }^{22}$ In their dynamic model of 'continuing conflict', Maxwell and Reuveny (2005) assume (and motivate) an analogous assumption of generational myopia. We conjecture the following result to be true in our model for a class of linear-homogeneous RLMs (formally introduced in Section 5.2): If the community residents are indeed extremely farsighted and cared sufficiently about the welfare of their distant progeny, then they will establish perfect property rights in every generation; alternatively, if each generation cares only a little about the welfare of its progeny, the poorer community can drag the richer community into anarchy when resource inequality is high and increasing over generations. We elaborate further on this issue in Section 5.3.

23 This implies that the community that desires weaker property rights can ensure that that regime gets chosen. A similar feature is present in Genicot and Skaperdas (2002). In Section 4.3, we argue that a partial relaxation of our assumption will not affect our results regarding the persistence of imperfect property rights.

${ }^{24}$ Without loss of generality, we take community 2 to be the resource-poorer community at the beginning (in equilibrium, this resource ranking will hold in all subsequent generations). The rights space $\Pi(1)$ can be thought of as follows: $\Pi(1) \equiv[\pi(0)-\varepsilon, \pi(0)+\eta] \cap[0,1]$, where $\pi(0)$ is the established rights regime in the (possibly notional) generation preceding our first generation. 
their predecessors. ${ }^{25}$ This process repeats itself unendingly, generation after generation.

In determining the 'equilibrium time-trajectories of property rights and resource inequality' in our (infinite) sequence-of-generations model, we proceed as follows. First, we determine the purestrategy subgame-perfect Nash equilibrium ( $S P N E)$ of the generational game in a particular period (by identifying the SPNE of the guns-and-butter game, and then the consequent optimal strategies in the rights negotiation game; see Section 4). Next, we 'piece together' the sequence of generational equilibria by using the transition rules determined by the resource law-of-motion and the institutional inertia parameters (see Section 5). It is our assumption of generational myopia that allows us to identify the equilibrium time trajectory vector $\left\{\pi^{*}(t), \rho^{*}(t)\right\}_{t=1}{ }^{\infty}$ in this manner.

\section{Illustrative Examples}

In this section, we illustrate the main results of the paper by identifying equilibrium outcomes in a set of parametric examples of our sequence-of-generations model : guns-and-butter choices in generational equilibria, and dynamic equilibrium evolution of property-rights.

We begin by focusing on the following 'single generation model':

Consider a single generation of members in the two communities, where the communities are asymmetrically resource-endowed - with $R_{1}>R_{2}>0$. Given their endowments, the communities first engage in a rights negotiation game (as described in Section 2.2) to collectively choose their rights regime $\pi$ over the 'full' rights space $\Pi=[0,1] .{ }^{26}$ Then, in the chosen rights regime $\pi$, the communities play their guns-and-butter game (as described in Section 2.1).

After studying some parametric examples of this single-generation model, we will move on to examples of a sequence-of-generations model. Note that such a dynamic model (with myopic residents) can be understood as an infinite sequence of single-generation models, linked across generations by the resource law-of-motion $F($.) and by the extent of institutional inertia $\{\varepsilon, \eta\}$. Our examples will clarify that the within-generation tension between creating and capturing value, and the across-generation evolution of property rights, depend crucially on the inequality in the resource endowments between the two communities in each generation, and on the resource law-of-motion

\footnotetext{
${ }^{25}$ In each generation $t$ in our model, the community residents participate in two negotiations: they first engage in the rights negotiation to establish $\pi(t)$, and then after producing guns and butter, they engage in peace negotiations to determine the butter bribe. If there existed a neutral third-party contract-enforcer in our model, a community could possibly link the two negotiations in the following way: "I promise to pay a high butter bribe to the other community in the future, provided that now the other community allows me to nudge the inherited property rights in a direction and to the extent that I prefer." We do not permit such linkages in our model, as we do not assume the existence of any external contract-enforcers.

${ }^{26}$ In this single-generation model, we assume that there is no institutional inertia (i.e., $\varepsilon=\eta=1$ ) so that the generation can choose its rights regime in an unconstrained manner over the $[0,1]$ space.
} 
that influences whether resource inequality across successive generations increases or decreases.

\subsection{Single Generation Examples}

We present a set of single-generation examples, in all of which we set $\sigma=1 / 2, \Pi=[0,1]$, and $R_{1}+R_{2}=1000$ with $R_{1}>R_{2}$. The examples differ in resource-distribution across the communities. In what follows, we present the equilibrium outcomes of the examples in Table 1, and discuss the forces generating the outcomes.

Table 1: Static Examples

\begin{tabular}{|c|c|c|c|c|c|c|}
\hline Example & resources & optimal $\pi$ choice & $\pi^{*}$ & $G_{i}^{*}$ & $b_{12} *$ & $W_{i}^{*}$ \\
\hline I & $\begin{array}{l}R_{1}=550 \\
R_{2}=450\end{array}$ & $\begin{array}{l}\text { community } 1: 1 \\
\text { community } 2: 1\end{array}$ & 1 & $\begin{array}{l}G_{1} *=G_{2} * \\
\quad=0\end{array}$ & 0 & $\begin{array}{l}W_{1}^{*}=550 \\
W_{2}^{*}=450\end{array}$ \\
\hline II & $\begin{array}{l}R_{1}=650 \\
R_{2}=350\end{array}$ & $\begin{array}{l}\text { community } 1: 1 \\
\text { community } 2: 0.65\end{array}$ & 0.65 & $\begin{array}{l}G_{1}^{*}=G_{2} * \\
=47.72\end{array}$ & 52.28 & $\begin{array}{c}W_{1} *=550 \\
W_{2}^{*}=354.55\end{array}$ \\
\hline III & $\begin{array}{l}R_{1}=750 \\
R_{2}=250\end{array}$ & $\begin{array}{l}\text { community } 1: 1 \\
\text { community } 2: 0\end{array}$ & 0 & $\begin{array}{l}G_{1}^{*}=G_{2} * \\
=166.67\end{array}$ & 250 & $\begin{array}{l}W_{1}^{*}=333.33 \\
W_{2}^{*}=333.33\end{array}$ \\
\hline IV & $\begin{array}{l}R_{1}=850 \\
R_{2}=150\end{array}$ & $\begin{array}{l}\text { community } 1: 1 \\
\text { community } 2: 0\end{array}$ & 0 & $\begin{array}{c}G_{1} *=166.23 \\
G_{2} *=150\end{array}$ & 333.11 & $\begin{array}{l}W_{1}^{*}=350.66 \\
W_{2}^{*}=333.11\end{array}$ \\
\hline
\end{tabular}

When resource inequality is quite small - as in Example I, with $\rho=0.45$ - both communities prefer maximal strengthening of property rights and collectively set $\pi^{*}=1 .{ }^{27}$ In the subsequent (trivial) guns-and-butter game, each community $i$ invests only in value creation and then consumes its created value. Such an efficient outcome, however, does not emerge for greater resource inequality. In contrast to Example I, Example III involves significant resource inequality, with $\rho=0.25$. In this case, there is complete divergence in $\pi$-preference between the communities - community 1 prefers $[\pi=1]$ to all other rights regimes while community 2 prefers $[\pi=0]$. Given the inherent veto power of the poorer community in the rights negotiation game, anarchy $[\pi=0]$ is the chosen regime. Sitting in anarchy, each community $i$ sets its guns investment to the common level $\{166.67\} .{ }^{28}$ In

27 The analysis in Section 4 can be employed to work out the static examples; we round off equilibrium values to the second decimal place. Note that in Example I, for any $\pi<1$, each community $i$ will set its guns investment at a common level $\{500[(1-\pi) /(3+\pi)]\}$ (and invest the remaining resources in butter). Then the peace agreement will require the richer community 1 to pay a butter bribe $\{50(1-\pi)\}$ to the poorer community 2. Straightforward calculations then establish that each community will want to set $\pi$ at 1 .

${ }^{28}$ Table 1 depicts the following: When resource inequality is below a threshold level both communities make identical investments in guns, while for resource inequality above the threshold the poorer community 
the subsequent peace agreement, community 1 pays a butter bribe of $\{250\}$ to community 2 , and as a result, the equilibrium butter consumption (and utility) of the communities are equalized. ${ }^{29}$ While the large resource inequality causes complete preference divergence between the communities and leads them to anarchy, each of the communities invest in butter and guns and are then situated in a 'Hobbesian equilibrium'. The defining feature of a Hobbesian equilibrium is that if peace negotiations were to fail, each community would attack the other to appropriate some rival butter. ${ }^{30}$ Given significant resource inequality, the only other scenario that the communities can find themselves is the following: the poorer community exercises its implicit veto power to establish anarchy and then invests all its resources in guns (with the sole aim of appropriating rival butter) while the richer community produces both guns and butter (with its guns being wholly defensive). Example IV - with high resource inequality $\rho=0.15$ - depicts such a 'Banditry equilibrium'. ${ }^{31}$

We conclude our discussion of the static examples by reverting back to a scenario of 'intermediate resource inequality': Example II, with $\rho=0.35$. In this case, there is significant but not complete divergence in $\pi$-preference between the communities: the poorer community's optimal choice is $\pi=0.65$ (while $\pi=1$ is optimal for the richer community). Following the establishment of $\pi=0.65$, the two communities play the guns-and-butter game in a way to generate a specific Hobbesian equilibrium outcome (as described in Table 1). Given that such intermediate resource inequality can lead to an 'intermediate rights regime $[\pi \in(0,1)]$ ', one can ask the following question: In a sequence-of-generations model, if the two communities start with such intermediate inequality in the first generation, then how will inter-generational decision-making cause the chosen rights regime as well as the guns-and-butter choices to evolve? In what follows, we address this question by studying a set of dynamic examples.

\subsection{Sequence- of-Generations Examples}

invests all its resources in guns. Further, $G_{2} * / G_{1} * \geq R_{2} / R_{1}$ (equivalently, $B_{2} * / B_{1} * \leq R_{2} / R_{1}$ ) and the poorer community 2 is always the recipient of a butter bribe. Despite having to bribe, community 1 always obtains a (weakly) higher payoff, while the phenomenon of Hirshleiferian paradox of power holds: $W_{2} * / W_{1} * \geq R_{2} / R_{1}$.

29 This outcome - in which payoffs of asymmetric contestants are equalized - has been dubbed in the extant literature as the strong form of the Hirshleiferian paradox of power. In our static examples, such an outcome will obtain whenever $\rho \in[1 / 6,5 / 18]$, anarchy being the chosen rights-regime for any such $\rho$.

${ }^{30}$ In a simpler guns-and-butter game in which peace negotiations are not permitted, Neary (1997) introduced the terminology of Hobbesian equilibria and Banditry equilibria - the former referring to a scenario where each player is a value-creator as well as a value-appropriator, and the latter referring to a scenario where one of the players specializes in value-appropriation (by being a bandit/predator).

${ }^{31}$ The following result holds in our single-generation model: When resource inequality is so high that the poorer community prefers to be a pure bandit, it also prefers anarchy. But for lesser resource inequality, the poorer community might prefer anarchy and then prefer to diversify (compare examples III and IV). 
In this subsection, we study a set of sequence-of-generations examples (see Section 2.2 for the model structure, and Section 5 for the equilibrium analysis), all of which incorporate a linear homogeneous resource law-of-motion $(R L M)$ of the form:

$$
F\left(R_{i}, B_{i}, W_{i}\right)=\left\{1+0.03\left[\lambda\left(B_{i} / R_{i}\right)+(1-\lambda)\left(W_{i} / R_{i}\right)\right]\right\} R_{i}, \text { with } \lambda \in(0,1) .^{32}
$$

In our first set of dynamic examples, we set $\sigma=1 / 2, R_{1}(1)=650, R_{2}(1)=350$. We determine the equilibrium trajectories of the chosen property rights regime $\left\{\pi^{*}(t)\right\}_{t=1}^{\infty}$, and the realized pergeneration resource inequality $\left\{\rho^{*}(t)\right\}_{t=1}^{\infty}$, for two different magnitudes of institutional inertia: (i) $\Pi(1)=[0,1]$ with $\varepsilon=\eta=1$, and (ii) $\Pi(1)=[0.19,0.21]$ with $\varepsilon=\eta=0.01$, and for two distinct structures of the linear RLM: structure [I] with $\lambda=0.4$, and structure [II] with $\lambda=0.8$.

Regarding the distinct inertia regimes, the simplest way to understand them is to assume that the generation preceding the first generation of our model had established the rights regime $\pi(0)=0.2$. In case (i) where there is no institutional inertia, this imposes no constraints on the rights space $[0,1]$ from which the first generation can choose $\pi(1)$, and such is the case for all successive generations. But in case (ii) the first generation has to choose $\pi(1)$ from the set $[0.19,0.21]$, and successive generations face a similar constraint, with the measure of $\Pi(t)$ being no greater than 0.02 for all $t$. It is also important to clarify the nature of the difference between $R L M$ structures [I] and [II]. Given that embodied human capital is the primary form of resource that we consider in our analysis, it is natural to posit that a community's resource growth depends positively on its aggregate butter consumption (capturing the positive impact of nutrition on human capital growth) and positively on the extent of its productive butter investment (capturing the positive effect of skill acquisition on human capital growth). The two RLM structures differ in the relative importance of one growthdriver $v s$. the other - as compared to structure [I], structure [II] posits current productive investment to be a more important driver of resource growth than current consumption.

\subsubsection{Equilibrium trajectories under no institutional inertia}

${ }^{32}$ Here, the per-period growth rate of resources of each community $i$ is $\left\{0.03\left[\lambda\left(B_{i} / R_{i}\right)+(1-\lambda)\left(W_{i} / R_{i}\right)\right]\right\}$. Note that if perfect property rights were exogenously imposed for perpetuity, in every period $t$ each community $i$ would specialize in butter investment and then consume own butter output (i.e., $W_{i}=B_{i}=R_{i}$ ); hence, each community's resources would grow at $3 \%$ per period irrespective of the value of $\lambda$. 


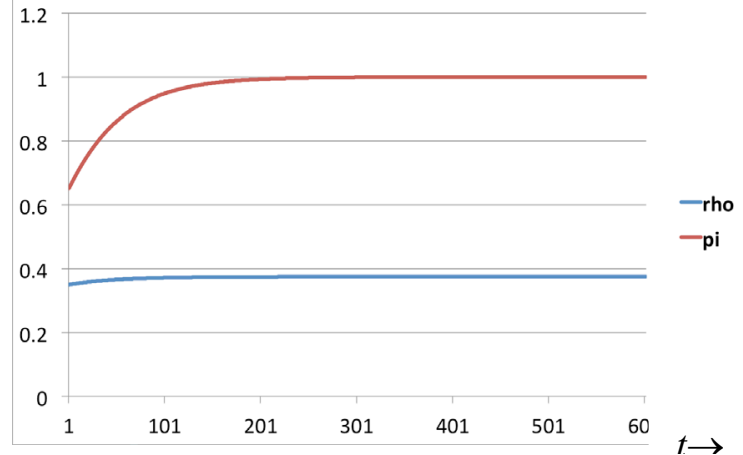

Figure 1A: $\lambda=0.4, \varepsilon=\eta=1$

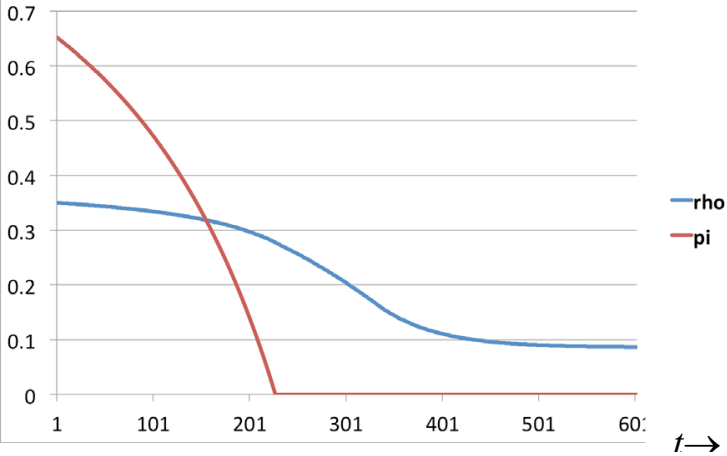

Figure 1B: $\lambda=0.8, \varepsilon=\eta=1$

The two panels of Figure 1 depict equilibrium time-trajectories of resource inequality $\{\rho(t)\}_{t=1}^{\infty}$ and property rights $\{\pi(t)\}_{t=1}^{\infty}$ under the two $R L M$ structures, when there is no institutional inertia. In the case where $\lambda=0.4$ (see Figure 1A), the within-generation resource inequality (which is initially at an intermediate level with $\rho(1)=0.35$ ) monotonically falls over time and eventually stabilizes 'near 0.4' (for all high values of $t, \rho^{*}(t)$ belongs in an $\varepsilon$-neighborhood of the constant value 0.375 ). The higher weight on past consumption in $R L M$ structure [I] promotes greater resource equality over time precisely because in every generation the consumption ratio $\left\{W_{2} / W_{1}\right\}$ is larger than the resource ratio $\left\{R_{2} / R_{1}\right\}$ while the production ratio $\left\{B_{2} / B_{1}\right\}$ is smaller than $\left\{R_{2} / R_{1}\right\}$. As resource inequality falls across generations, the optimal property rights choice of the poorer community rises. That induces the two communities to continually strengthen property rights so that $\pi^{*}(t) \rightarrow 1$. $^{33}$ Figure 1B shows that the equilibrium trajectories $\left\{\rho^{*}(t)\right\}_{t=1}^{\infty}$ and $\left\{\pi^{*}(t)\right\}_{t=1}^{\infty}$ are very different under the $R L M$ structure [II] with $\lambda=0.8$, where past productive investment is the relatively more important driver of resource growth. In this case, in the dynamic equilibrium, resource inequality starts to rise over generations from the initial intermediate level. Along with the rise in inequality, the poorer community 2 ensures that the chosen rights regime gets continually weaker till anarchy is permanently established. As this process unfolds, at some point community 2 becomes a bandit, and then it remains a bandit forever after. Does this bandit community eventually vanish? Not necessarily. As Figure 1B shows, the resource inequality stabilizes in the long run in our example: $\rho^{*}(t)$ converges to a relatively small but strictly positive value smaller than 0.1 .

\subsubsection{Equilibrium trajectories under institutional inertia}

${ }^{33}$ In our model, when successive generations continually raise $\pi$, perfect property rights $[\pi=1]$ may or may not be exactly attained. Note that in Figure $1 \mathrm{~A}, \pi^{*}(t)$ monotonically increases but only asymptotes to unity, while it attains unity in Figure 2A. For an explanation, see the discussion in footnote 49 in Section 5.1. 


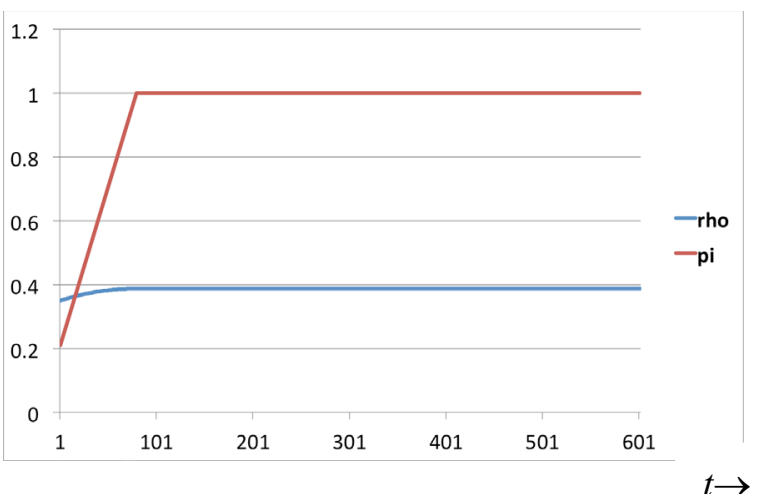

Figure 2A: $\lambda=0.4, \varepsilon=\eta=0.01$

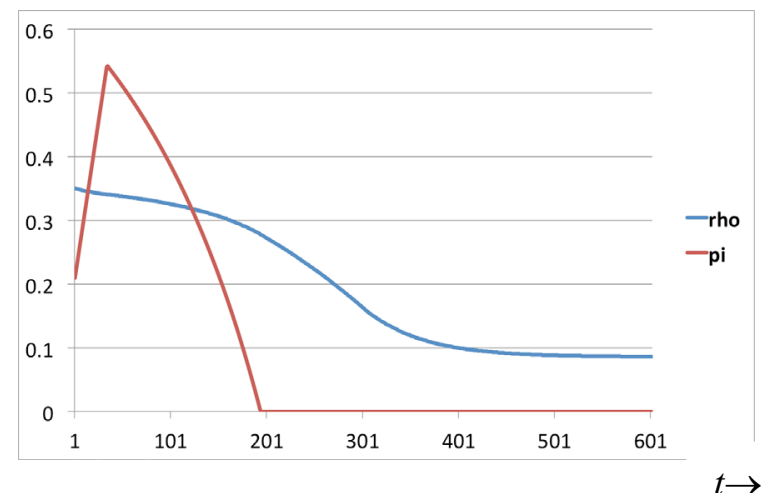

Figure 2B: $\lambda=0.8, \varepsilon=\eta=0.01$

The two panels of Figure 2 show the equilibrium trajectories of resource inequality and property rights under the two $R L M$ structures described above, but under the assumption that significant institutional inertia limits the nudge that each generation can give to its inherited rights regime. Note the striking similarity in the long-run between Figures 1A and 2A (which incorporate RLM structure $[\mathrm{I}]$ with $\lambda=0.4$ ), and between Figures $1 \mathrm{~B}$ and $2 \mathrm{~B}$ (which incorporate the $R L M$ structure [II] with $\lambda=0.8$ ). As we establish analytically in Section 5 , there are many parameter configurations of our model where the extent of institutional inertia has little effect on the long-run trajectories $\left\{\rho^{*}(t)\right\}_{t=1}^{\infty}$ and $\left\{\pi^{*}(t)\right\}_{t=1}^{\infty}$ - especially regarding whether anarchy or perfect property rights are eventually and perpetually established. ${ }^{34}$

There can however be interesting differences in the short-run (as a comparison of Figures $1 \mathrm{~B}$ and $2 \mathrm{~B}$ shows). In the case where $\lambda=0.8$, the chosen rights regime falls monotonically when there is no institutional inertia. But $\pi^{*}(t)$ initially rises and eventually falls when considerable institutional inertia is present - thus depicting possible non-monotonicity in the evolution of property rights.

\subsubsection{Dynamic evolution of welfare}

Apart from the evolution of property rights and inequality, it is of interest to understand how community welfare evolves in the dynamic equilibria of our sequence-of-generations model. To that end, we compare two dynamic examples A and B. Example A is one that we have studied above, with $\sigma=1 / 2, R_{1}(1)=650, R_{2}(1)=350$, and $\Pi(1)=[0,1]$ with $\varepsilon=\eta=1$ (and whose equilibrium trajectories $\left\{\rho^{*}(t)\right\}_{t=1}^{\infty}$ and $\left\{\pi^{*}(t)\right\}_{t=1}^{\infty}$ are shown in Figure 1). For example B, we set $\sigma=1 / 2, R_{1}(1)=830, R_{2}(1)=170$, and $\Pi(1)=[0,1]$ with $\varepsilon=\eta=1$. So the examples (which ignore institutional inertia) differ in the extent of initial resource inequality between the communities. ${ }^{35}$

\footnotetext{
${ }^{34}$ Having said that, we note that Proposition 12 in Section 5 identifies a scenario where it is precisely the presence of institutional inertia that causes the two communities to devolve to perpetual anarchy.

${ }^{35}$ The equilibrium trajectories of resource inequality and property rights in example B share the same features of that in example A. Specifically, perfect property rights are attained in the long-run under $R L M$
} 
Table 2A : Dynamic Example A

\begin{tabular}{|c|c|c|c|c|c|c|c|c|c|c|}
\hline & & $t=1$ & $t=25$ & $t=50$ & $t=75$ & $\cdots$ & $t=250$ & $t=500$ & $t=750$ & $t=1000$ \\
\hline \multirow{2}{*}{$W_{1} *(t)$} & $\lambda^{\prime}=0.8$ & $\mathbf{5 5 0}$ & 1026 & 1940 & 3618 & & $1.3 \times 10^{5}$ & $3.2 \times 10^{7}$ & $8.9 \times 10^{9}$ & $2.5 \times 10^{12}$ \\
\cline { 2 - 11 } & $\lambda^{\prime \prime}=0.4$ & $\mathbf{5 5 0}$ & $\mathbf{1 1 1 5}$ & $\mathbf{2 3 2 7}$ & $\mathbf{4 8 5 9}$ & & $\mathbf{8 . 5} \times \mathbf{1 0}^{\mathbf{5}}$ & $\mathbf{1 . 4 \times 1 0 ^ { 9 }}$ & $\mathbf{2 . 2} \times \mathbf{1 0}^{\mathbf{1 2}}$ & $\mathbf{3 . 6}^{\mathbf{2}} \times \mathbf{1 0}^{\mathbf{1 5}}$ \\
\hline \multirow{2}{*}{$W_{2}^{*}(t)$} & $\lambda^{\prime}=0.8$ & $\mathbf{3 5 5}$ & 668 & 1282 & 2432 & & $1.3 \times 10^{5}$ & $2.4 \times 10^{7}$ & $6.7 \times 10^{9}$ & $1.9 \times 10^{12}$ \\
\cline { 2 - 11 } & $\lambda^{\prime \prime}=0.4$ & $\mathbf{3 5 5}$ & $\mathbf{6 9 6}$ & $\mathbf{1 4 2 7}$ & $\mathbf{2 9 5 3}$ & & $\mathbf{5 . 1 \times 1 0 ^ { \mathbf { 5 } }}$ & $\mathbf{8 . 3} \times \mathbf{1 0}^{\mathbf{8}}$ & $\mathbf{1 . 3}^{\mathbf{2}} \times \mathbf{1 0}^{\mathbf{1 2}}$ & $\mathbf{2 . 2} \times \mathbf{1 0}^{\mathbf{1 5}}$ \\
\hline
\end{tabular}

structure [I], while the rights regime devolves to perpetual anarchy under $R L M$ structure [II]. 
Table 2B : Dynamic Example B

\begin{tabular}{|c|c|c|c|c|c|c|c|c|c|c|}
\hline & & $t=1$ & $t=25$ & $t=50$ & $t=75$ & $\cdots$ & $t=250$ & $t=500$ & $t=750$ & $t=1000$ \\
\hline \multirow{2}{*}{$W_{1}^{*}(t)$} & $\lambda^{\prime}=0.8$ & $\mathbf{3 3 3}$ & $\mathbf{5 8 2}$ & $\mathbf{1 0 4 3}$ & 1861 & & $9.9 \times 10^{4}$ & $2.8 \times 10^{7}$ & $7.9 \times 10^{9}$ & $2.2 \times 10^{12}$ \\
\cline { 2 - 11 } & $\lambda^{\prime \prime}=0.4$ & $\mathbf{3 3 3}$ & 536 & 880 & $\mathbf{1 9 6 9}$ & & $\mathbf{3 . 5} \times \mathbf{1 0}^{\mathbf{5}}$ & $\mathbf{5 . 7 \times 1 0 ^ { 8 }}$ & $\mathbf{9 . 2 \times 1 0 ^ { 1 1 }}$ & $\mathbf{1 . 5} \times \mathbf{1 0}^{\mathbf{1 5}}$ \\
\hline \multirow{2}{*}{$W_{2}^{*}(t)$} & $\lambda^{\prime}=0.8$ & $\mathbf{3 3 3}$ & $\mathbf{5 4 0}$ & $\mathbf{9 0 6}$ & $\mathbf{1 5 4 1}$ & & $7.5 \times 10^{4}$ & $2.1 \times 10^{7}$ & $5.9 \times 10^{9}$ & $1.7 \times 10^{12}$ \\
\cline { 2 - 10 } & $\lambda^{\prime \prime}=0.4$ & $\mathbf{3 3 3}$ & 536 & 880 & 1520 & & $\mathbf{2 . 1 \times 1 0 ^ { 5 }}$ & $\mathbf{3 . 4 \times 1 0 ^ { 8 }}$ & $\mathbf{5 . 5 \times 1 0 ^ { 1 1 }}$ & $\mathbf{8 . 9 \times 1 0 ^ { \mathbf { 1 4 } }}$ \\
\hline
\end{tabular}

Tables $2 \mathrm{~A}$ and $2 \mathrm{~B}$ depict the equilibrium welfare levels of the two communities $\left\{W_{1} *(t), W_{2} *(t)\right\}$ for different generations $t$ under $R L M$ structures [I] and [II]. The tables clarify the following possibilities. ${ }^{36}$ For different levels of initial resource inequality (covering both examples A and B), in the long-run (for all $t>100$ in our examples) it is the case that that $R L M$ structure that ensures attainment of perfect property rights (with $\lambda=0.4$ ) is also associated with higher community welfare for each of the communities (as compared to the $R L M$ structure with $\lambda=0.8$ that causes devolution to anarchy). In contrast, short-run equilibrium outcomes can be 'contradictory' depending upon the structural parameters and initial conditions. Specifically, in the short-run (for $t \leq 50$ ) in example B, where there is significant initial resource inequality, it is the $R L M$ structure [I] with $\lambda=0.4$ that is associated with higher community welfare for each of the communities (as compared to structure [II] with $\lambda=0.8$ ). This is not the case in example A with smaller initial resource inequality, where the rights-promoting RLM structure [I] generates better welfare outcomes in the short-run as well. These findings convey an important message: While a continual movement of a multi-community system (where generational decisions are made myopically) towards stronger property-rights regimes can indeed ensure long-run welfare gains vis-à-vis a system that devolves to anarchy, it is not guaranteed that all generations will be better off in the former system than in the latter - some generations in the near-term might be better off in the latter system. In Section 5.3, we will further study this issue and discuss the merits of a policy intervention by a social planner.

\section{Generational Decision - Making}

In our sequence-of-generations model, each generation $t$ residents of the two communities make the following decisions: First, they collectively choose their property rights regime $\pi(t)$ from their inherited rights space $\Pi(t)$ via the rights negotiation game. Then, given their inherited resources $\left\{R_{1}(t), R_{2}(t)\right\}$ and their chosen rights regime $\pi(t)$, the two generation $t$ communities play the guns-

\footnotetext{
${ }^{36}$ To enhance readability, consumption quantities less than 10,000 have been rounded off to the nearest integer and consumption quantities greater than 10,000 have been rounded off to two significant figures. We have also bolded the higher welfare value for comparison - i.e., for each community in each generation.
} 
and-butter game. In what follows, we first characterize the subgame-perfect Nash equilibrium $(S P N E)$ of the guns-and-butter game. ${ }^{37}$ We then identify the equilibrium property rights regime that will be chosen by generation $t$ residents before engaging in value-creation and value-capture.

\subsection{The Guns - and - Butter Equilibrium}

In this subsection, we determine the $S P N E$ outcome of the guns-and-butter game in a particular generation (say, $t$ ), given the resource endowments of communities 1 and 2: $R_{1} \geq R_{2}>0$, and the established rights regime $\pi \in[0,1]$. Note that if property rights are perfect $(\pi=1)$, then in the unique equilibrium, each community will invest all its resources in butter production and consume all the butter that it produces. In what follows, we focus on the case where $\pi<1$.

We begin by studying the terminal conflict that would occur if peace negotiations were to break down. Consider the scenario where the resource investments of the two communities have led to a guns-and-butter vector $\left\{\left(G_{1}, B_{1}\right),\left(G_{2}, B_{2}\right)\right\}$ to be realized. Then Lemma 1 identifies the equilibrium attack-defense split of the guns output of each community, and their equilibrium continuation payoffs, if the communities fail to negotiate peace and so enter into a terminal conflict.

LEMMA 1. Take as given $\sigma \in[1 / 2,1), \pi \in[0,1)$, and a guns-and-butter vector $\left\{\left(G_{1}, B_{1}\right),\left(G_{2}, B_{2}\right)\right\}$ such that $B_{1}+B_{2}>0$ and $G_{1} \times G_{2}>0 .{ }^{38}$ Suppose that peace-negotiations have failed and no buttertransfer has taken place. Then for $i, j=1,2$, and $i \neq j$, the attack-defense split of each community $i$ in the unique continuation SPNE of the terminal conflict phase will be:

$$
\begin{aligned}
& \left\{A_{i}^{\dagger}=\left[B_{j} /\left(B_{i}+B_{j}\right)\right] G_{i} ; D_{i}^{\dagger}=\left[B_{i} /\left(B_{i}+B_{j}\right)\right] G_{i}\right\} \text { if } B_{i}>0 \text { and } B_{j}>0 ; \text { and } \\
& \left\{A_{i}^{\dagger}=0 ; D_{i}^{\dagger}=G_{i}\right\} \text { and }\left\{A_{j}^{\dagger}=G_{j} ; D_{j}^{\dagger}=0\right\} \text { if } B_{i}>0 \text { and } B_{j}=0 .
\end{aligned}
$$

In either case, the equilibrium continuation payoff to each community $i$ (for $i=1,2$ ) will be: $\pi B_{i}+$ $\left\{(1-\pi)\left[G_{i} /\left(G_{1}+G_{2}\right)\right]\right\} \cdot \sigma\left[B_{1}+B_{2}\right]$.

If a community produces no butter, then it will be a pure bandit that will use its guns for offense (while its rival will use all its guns in defense). Lemma 1 establishes that if neither community is a pure bandit, then the attack-defense split of each community's guns will be determined by the ratio of rival butter production to own butter production.

\footnotetext{
${ }^{37}$ We restrict attention to pure-strategy $S P N E$ in our analysis. As our subsequent results show, there always exists a unique pure-strategy $S P N E$ outcome in the guns-and-butter game and the rights negotiation game.

${ }^{38}$ We will subsequently argue that any $S P N E$ of the guns-and-butter game (given $\pi<1$ ) must have $B_{1}+B_{2}>0$ and $G_{1} \times G_{2}>0$. For completeness, we note that in the continuation SPNE in the terminal conflict phase (after the failure of peace-negotiations): if $B_{1}=B_{2}=0$ then the payoff to each community $i$ will be zero; if $G_{1}=G_{2}=0$ then the payoff to each community $i$ will be $B_{i}$; and if $G_{i}>0$ and $G_{j}=0$ then the payoff to community $i$ will be $\left\{B_{i}+(1-\pi) \sigma B_{j}\right\}$ and that to community $j$ will be $\pi B_{j}$.
} 
Using Lemma 1, we determine the Nash Bargaining Solution of the peace negotiation process. Given $\sigma \in[1 / 2,1)$ and $\pi \in[0,1)$, for a guns-and-butter vector $\left\{\left(G_{1}, B_{1}\right),\left(G_{2}, B_{2}\right)\right\}$ with $B_{1}+B_{2}>0$ and $G_{1} \times G_{2}>0$, the 'gross surplus' to be bargained over will be $\left[B_{1}+B_{2}\right]$, while the 'outside option' of each community $i$ will be: $\pi \cdot B_{i}+\left\{(1-\pi)\left[G_{i} /\left(G_{1}+G_{2}\right)\right]\right\} . \sigma\left[B_{1}+B_{2}\right]$. Given that, the Nash Bargaining Solution will involve one community giving a butter bribe to the other such that each community $i$ 's final payoff becomes \{half of net bargaining surplus + own outside option $\} \equiv$ $0.5\left\{(1-\pi)(1-\sigma)\left(B_{1}+B_{2}\right)\right\}+\left\{\pi \cdot B_{i}+(1-\pi)\left[G_{i} /\left(G_{1}+G_{2}\right)\right] \sigma\left[B_{1}+B_{2}\right]\right\} .{ }^{39}$

The nature of the guns-and-butter equilibrium will depend on the extent of resource inequality between the communities as measured by $\rho \equiv R_{2} / R \in(0,1 / 2]$. We define the critical fraction:

$$
\tilde{\rho}(\pi \mid \sigma)=0.5\{[(1-\pi) \sigma] /[1+\pi+(1-\pi) \sigma]\} .
$$

Note that $\tilde{\rho}(\pi \mid \sigma) \in(0,0.5 \sigma /(1+\sigma)]<1 / 4$, and falls from $0.5 \sigma /(1+\sigma)$ to 0 as $\pi$ rises from 0 to 1 . Our next result shows that given any $\pi<1$, if there is limited resource inequality between the communities such that $\rho \in(\tilde{\rho}(\pi \mid \sigma), 1 / 2]$, then the communities will be situated in a Hobbesian equilibrium - with $A_{1}^{\dagger}>0$ and $A_{2}^{\dagger}>0$ - where, if peace negotiations fail then each community will launch an appropriative attack on its rival. In contrast, if there is significant resource inequality such that $\rho \in(0, \tilde{\rho}(\pi \mid \sigma)]$, then the communities will find themselves in a Banditry equilibrium - with $A_{1}^{\dagger}=0$ and $A_{2}^{\dagger}=G_{2}-$ in which the poorer community 2 will be a pure bandit.

Proposition 2: Take as given $\sigma \in[1 / 2,1)$, and $R_{1} \geq R_{2}>0$, with $R \equiv R_{1}+R_{2}$ and $\rho \equiv R_{2} / R$.

[A] If $\pi<1$, in the unique $S P N E$ of the guns-and-butter game community 2's guns investment is:

$$
G_{2}^{\dagger}(\sigma, \pi, R, \rho)= \begin{cases}\tilde{\rho}(\pi \mid \sigma) \cdot R & \text { for } \rho \in(\tilde{\rho}(\pi \mid \sigma), 1 / 2], \\ \rho . R & \text { for } \rho \in(0, \tilde{\rho}(\pi \mid \sigma)]\end{cases}
$$

while community 1 's guns investment is:

$$
G_{1}^{\dagger}(\sigma, \pi, R, \rho)= \begin{cases}\tilde{\rho}(\pi \mid \sigma) \cdot R & \text { for } \rho \in(\tilde{\rho}(\pi \mid \sigma), 1 / 2], \\ \{\sqrt{[4 \cdot \tilde{\rho}(\pi \mid \sigma) \cdot \rho]}-\rho\} \cdot R & \text { for } \rho \in(0, \tilde{\rho}(\pi \mid \sigma)] .\end{cases}
$$

For each community, the remaining resources are invested in butter production, i.e., $B_{i}^{\dagger}(\sigma, \pi, R, \rho)=$ $R_{i}-G_{i}^{\dagger}(\sigma, \pi, R, \rho)$ for $i=1,2$. In the successful peace negotiations, the richer community 1 gives the poorer community 2 a strictly positive butter bribe of:

$$
b_{12}{ }^{\dagger}(\sigma, \pi, R, \rho)=0.5(1-\pi)\left\{\left[B_{1}^{\dagger}(.)-B_{2}^{\dagger}(.)\right]-\sigma\left[\left(B_{1}^{\dagger}(.)+B_{2}^{\dagger}(.)\right) /\left(G_{1}^{\dagger}(.)+G_{2}^{\dagger}(.)\right)\right]\left(G_{1}^{\dagger}(.)-G_{2}^{\dagger}(.)\right)\right\} .
$$

The unique $S P N E$ payoffs of the two communities (equivalently, their equilibrium aggregate butter

${ }^{39}$ In the continuation $S P N E$ in the peace negotiation process: the net bargaining surplus will be zero if either $B_{1}=B_{2}=0$ or $G_{1}=G_{2}=0$, while it will be $(1-\pi)(1-\sigma) B_{j}$ if $G_{i}>0$ and $G_{j}=0$. 
consumptions), denoted by $W_{1}^{\dagger}(\sigma, \pi, R, \rho)$ and $W_{2}^{\dagger}(\sigma, \pi, R, \rho)$, are:

$$
\begin{aligned}
& W_{1}^{\dagger}(.)= \begin{cases}\left\{(1-\rho)+\frac{[1-\pi][1+(1-\sigma) \pi]}{2[1+\sigma+(1-\sigma) \pi]}\right\} \cdot R & \text { for } \rho \in(\tilde{\rho}(\pi \mid \sigma), 1 / 2],\end{cases} \\
& W_{2}^{\dagger}(.)= \begin{cases}\left\{\pi \rho+\frac{[1-\pi][1+(1-\sigma) \pi]}{2[1+\sigma+(1-\sigma) \pi]}\right\} \cdot R & \text { for } \rho \in(0, \tilde{\rho}(\pi \mid \sigma)] ; \\
\left\{[\pi+(1-\pi) \sigma] \sqrt{\left[\frac{2(1-\pi) \sigma \rho}{1+\pi+(1-\pi) \sigma}\right]}+\frac{(1-\pi)(1-\sigma)}{2}-(1-\pi) \sigma \rho\right\} \cdot R & \text { for } \rho \in(0, \tilde{\rho}(\pi \mid \sigma)] .\end{cases}
\end{aligned}
$$

[B] If $\pi=1$, then each community produces no guns and consumes all the butter that it produces: for $i=1,2, G_{i}^{\dagger}(\sigma, \pi=1, R, \rho)=0$ and $W_{i}^{\dagger}(\sigma, \pi=1, R, \rho)=B_{i}^{\dagger}(\sigma, \pi=1, R, \rho)=R_{i}\left(\right.$ and $\left.b_{12}{ }^{\dagger}()=0.\right)$.

It is immediate from Proposition 2 that if the two communities happen to be symmetric with $R_{1}=R_{2}$, the unique $S P N E$ will be symmetric in terms of payoffs as well as behavior (with zero butter bribe) in any rights regime $\pi \in[0,1]$. In contrast, when the two communities are asymmetric with $R_{1}>R_{2}$, the proposition allows us to identify the important differences in their behavior and payoffs when property rights are imperfect. We conclude this subsection by highlighting these differences.

Corollary 3: Given $\sigma \in[1 / 2,1), R \equiv R_{1}+R_{2}>0$, and $\rho \equiv R_{2} / R \in(0,1 / 2)$, the unique $S P N E$ of the guns-and-butter game exhibits the following properties for all $\pi \in[0,1)$.

[A] For all $\rho \equiv R_{2} / R \in(0,1 / 2):\left[W_{1}^{\dagger}(\sigma, \pi, R, \rho)+W_{2}^{\dagger}(\sigma, \pi, R, \rho)\right]$ strictly increases in $\pi$; and $\left[W_{2}^{\dagger}(.) / R_{2}\right]>\left[W_{1}^{\dagger}(.) / R_{1}\right], b_{12}^{\dagger}(\sigma, \pi, R, \rho)>0$, and $\partial W_{1}^{\dagger}(.) / \partial \pi>\max \left\{0, \partial W_{2}^{\dagger}(.) / \partial \pi\right\}$.

[B] In addition, defining $\rho^{-}(\sigma) \equiv\left[1 / 2-\left\{\sigma /(1+\sigma)^{2}\right\}\right] \in(0,0.5)$, for all $\rho \equiv R_{2} / R \in\left(0, \rho^{-}(\sigma)\right)$ : $W_{2}^{\dagger}\left(\right.$.) strictly falls in $\pi$, and $\left[W_{2}^{\dagger}(.) / R_{2}\right]>1>\left[W_{1}^{\dagger}(.) / R_{1}\right]$.

It is worth emphasizing the following imports of Corollary 3 when $R_{1}>R_{2}$ and $\pi<1$. Overall efficiency (as measured by the sum of payoffs of the communities) unambiguously increases with the strengthening of property rights. ${ }^{40}$ Further, for all levels of resource inequality (i.e., irrespective of whether the Hobbesian or the Banditry equilibrium obtains), three equilibrium features go hand in

${ }^{40}$ Extant research has shown that the specific way of modeling the phenomenon of 'strengthening property rights' determines whether overall efficiency will be monotonically increasing in property rights or not. Gonzalez (2007) shows that this relationship need not be monotonic when strengthening property rights is equivalent to making 'appropriative attacks less effective'. In contrast (and as is the case in our model), when strengthening property rights implies increasing the security parameter $\pi$, Yoo (2013) clarifies that overall efficiency will rise in $\pi$. Thus, in our sequence-of-generation model, myopic generations can choose to weaken inherited property rights even when strengthening these rights would increase overall efficiency. 
hand: $(a)$ the Hirshleiferian paradox of power holds 'under the shadow of conflict' with $\left[W_{2}^{\dagger}(.) / R_{2}\right]$ $>\left[W_{1}^{\dagger}(.) / R_{1}\right],(b)$ the poorer community 2 is conflict-prone in that it acquiesces to peace only upon receiving a butter bribe $b_{12}{ }^{\dagger}()>$.0 , and (c) while the richer community prefers to strengthen property rights, the poorer community has a weaker preference in this regard: $\partial W_{1}^{\dagger} / \partial \pi>\max \left\{0, \partial W_{2}^{\dagger} / \partial \pi\right\}$.

But more importantly for our subsequent analysis, Corollary 3 clarifies that not only does the poorer community 2 prefer worse property rights in relative terms (which is the case for all levels of resource asymmetry), it strictly prefers an absolute decline in the property rights regime when the resource-inequality between the contestants is large. Specifically, when $\rho \equiv R_{2} / R$ is less than $\rho^{-}(\sigma)$, there is complete divergence in the induced $\pi$-preferences of the two communities, with $\partial W_{2}^{\dagger}(\pi) / \partial \pi$ $<0<\partial W_{1}^{\dagger}(\pi) / \partial \pi^{41}$ It is this phenomenon that thwarts the attainment of perfect property rights in the long-run when successive generations of residents interact over time.

\subsection{Rights Negotiation in the Single Generation Model}

We now turn to the rights negotiation game that precedes the guns-and-butter game. After having realized their resource endowments $\left\{R_{1}, R_{2}\right\}$ and inherited the rights space $\Pi(.) \subseteq[0,1]$, the two communities negotiate about their collective property rights choice. In this subsection, we will take $R \equiv R_{1}+R_{2}>0$ with $\rho \equiv R_{2} / R \in(0,1 / 2)$, and will consider the case where $\Pi()=.[0,1]$ (i.e., where the communities are unconstrained in choosing $\pi$ ). Recognize that by doing so, we are focusing on the single-generation model that we defined in Section 3. Identifying the collectively chosen rights regime in this static model will help us study the sequence-of-generations model in Section 5.

In the single-generation model, the rights regime is established costlessly and with mutual consent as follows: first community 1 announces a number $s_{1} \in[0,1]$ and then community 2 announces $s_{2} \in[0,1]$; given the announcements $\left\{s_{1}, s_{2}\right\}$ the property rights enforcement level $\pi=\min \left\{s_{1}, s_{2}\right\}$ is established. Given the induced preferences of the communities over property rights, as embodied in their payoff functions $W_{1}^{\dagger}(\sigma, \pi, R, \rho)$ and $W_{2}^{\dagger}(\sigma, \pi, R, \rho)$ in the guns-and-butter game, what will be the equilibrium property rights choice in this scenario? The answer depends on the extent of resource inequality between the communities.

For any $\sigma \in[1 / 2,1)$, we define the following 'resource inequality cutoffs': $\rho^{-}(\sigma) \equiv\left[1 / 2-\left\{\sigma /(1+\sigma)^{2}\right\}\right]$, and $\rho^{+}(\sigma) \equiv[1 / 2-\sigma / 4]$. Note that $0<\tilde{\rho}(\pi \mid \sigma)<\rho^{-}(\sigma)<\rho^{+}(\sigma)<1 / 2$ for $\sigma \in[1 / 2,1)$ and $\pi<1$. Further, define:

$$
\widetilde{\pi}(\rho \mid \sigma)=\left[\{\sigma /(1 / 2-\rho)\}^{1 / 2}-(1+\sigma)\right] /[1-\sigma] .
$$

Note that $\widetilde{\pi}(\rho \mid \sigma) \in(0,1)$ for all $\rho \in\left(\rho^{-}(\sigma), \rho^{+}(\sigma)\right)$, and it strictly increases from 0 to 1 as $\rho$

41 The following results also hold in the guns-and-butter equilibrium when $R_{1}>R_{2}$ : (i) it is always the case that $\left\{B_{1}{ }^{*}>B_{2}{ }^{*}\right\},\left\{G_{1}{ }^{*} \geq G_{2}{ }^{*}\right\}$, and $\left\{W_{1}{ }^{*} \geq W_{2} *\right\}$, (ii) the butter bribe amount $b_{12}{ }^{\dagger}$ (.) strictly decreases in $\pi$, and (iii) when $\rho<\rho^{-}(\sigma)$ a stronger version of the Hirshleiferian paradox of power holds: $W_{2}^{\dagger}(.) / R_{2}>1>W_{1}^{\dagger}(.) / R_{1}$. 
increases from $\rho^{-}(\sigma)$ to $\rho^{+}(\sigma)$.

Proposition 4: Given $\sigma \in[1 / 2,1), \Pi=[0,1]$, and $R \equiv R_{1}+R_{2}>0$ with $\rho \equiv R_{2} / R \in(0,1 / 2)$, the unique $S P N E$ outcome in the rights negotiation game in the single-generation model is the establishment of the regime $\pi^{\dagger}(\rho \mid \sigma)$ which maximizes $W_{2}^{\dagger}(\sigma, \pi, R, \rho)$ over $\pi \in[0,1]$ and is given by:

$$
\pi^{\dagger}(\rho \mid \sigma)= \begin{cases}0 & \text { for } \rho \in\left(0, \rho^{-}(\sigma)\right], \\ \widetilde{\pi}(\rho \mid \sigma) & \text { for } \rho \in\left(\rho^{-}(\sigma), \rho^{+}(\sigma)\right), \\ 1 & \text { for } \rho \in\left[\rho^{+}(\sigma), 1 / 2\right) .{ }^{42}\end{cases}
$$

The poorer community faces the following trade-off in identifying its optimal rights regime: weaker property rights permits greater appropriation of rival butter, while stronger property rights limits the extent of inefficient investment in guns. When community 2 is relatively well-off then the second effect dominates the first and it desires strengthening of inherited $\pi$; but when it has limited resources then the first effect dominates, and in the extreme (when $\rho \leq \rho^{-}(\sigma)$ ) it desires $\pi=0$ so that the maximum amount of rival butter is contestable. Consequently, when resource-inequality is small (resp., large) $W_{2}^{\dagger}$.) rises (resp., falls) monotonically in $\pi$ leading to $\pi^{\dagger}\left(\right.$.) $=1$ (resp., $\pi^{\dagger}($.) $=0$ ), and in case of intermediate inequality, $W_{2}^{\dagger}($.$) is uniquely maximized at an 'interior' regime \widetilde{\pi}(\rho \mid \sigma)$.

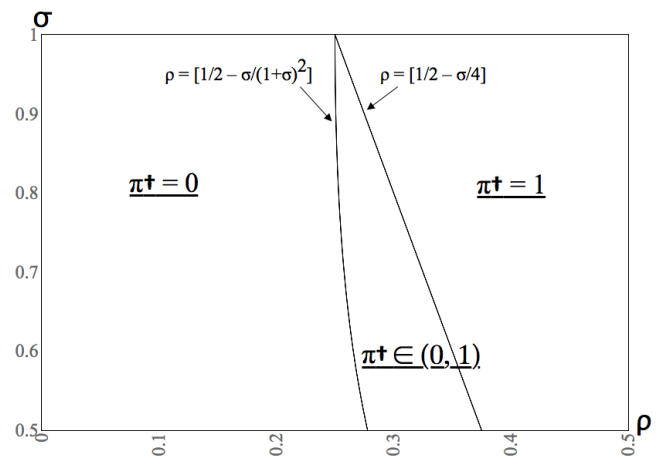

Figure 3A

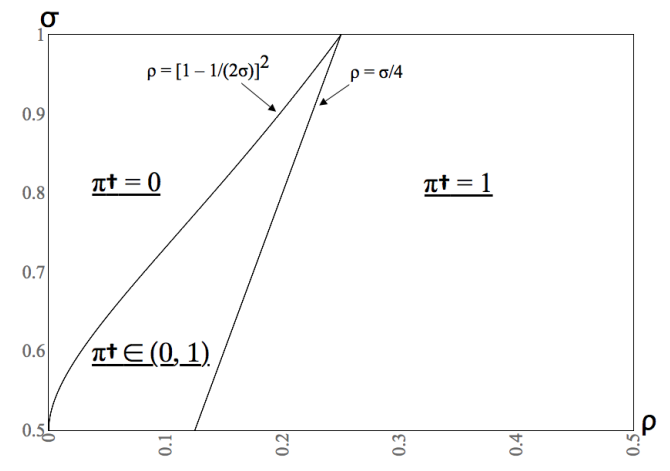

Figure 3B

In the single-generation model, property rights negotiations are so structured that the equilibrium outcome coincides with the poorer community 2's optimal property-rights choice. Figure 3A shows the property rights choices for different $\{\rho, \sigma\}$ values in the single-generation model.

\subsection{Robustness Checks}

The possibility that generational rights negotiations can fail to strengthen inherited weak property rights arises principally from two features of our model: $(a)$ when resource inequality is high the

42 The stated outcome will also be the unique SPNE outcome in the rights negotiation game if community 2 makes the first announcement and community 1 the second; it will also be the unique outcome of 'Nash equilibrium in undominated strategies' if the two communities make simultaneous announcements. 
poorer community strictly prefers a decline in property rights, and $(b)$ in the rights negotiation game, the community with the weaker $\pi$-preference can ensure the selection of its most-preferred rights regime. We now discuss the robustness of these features in particular, and the singlegeneration results in general, in a few variants of our model.

We begin by studying the robustness of preference divergence between the richer and the poorer communities under two alternative scenarios. First, we consider the issue of commitment in peace negotiations. Note that a peace treaty in the guns-and-butter game necessarily requires each community to commit its army solely to defense. Our analysis proceeds under the assumption that such commitments are credible, with there being no possibility for any community to renege and (surreptitiously) deploy a part of its army to offense. In what follows, we briefly discuss what would happen in the single-generation model in a 'no-commitment scenario' - where communities cannot commit to peace even if they have signed a peace treaty. Note that when it is common knowledge that the communities cannot credibly commit to peace, the guns-and-butter game will in effect skip Stage 2 and directly enter the terminal conflict phase in Stage 3 after Stage 1. We refer to this as the 'guns-and-butter game under no commitment'. For our single-generation model that concludes with such a guns-and-butter game, the following result holds.

PROPOSITION 5: Take as given $\sigma \in[1 / 2,1), \Pi=[0,1]$, and $\left\{R_{1}, R_{2}\right\}$ such that $\rho \equiv\left[R_{2} /\left(R_{1}+R_{2}\right)\right] \in(0,1 / 2)$. Consider the single-generation model in which rights negotiation game is followed by the guns-andbutter game under no commitment. The unique SPNE outcome in the rights negotiation game will lead to the establishment of the property rights regime $\pi^{[N]}(\rho \mid \sigma)$, where

$$
\pi^{[N]}(\rho \mid \sigma)= \begin{cases}0 & \text { for } \rho \in\left(0,(1-\{1 /(2 \sigma)\})^{2}\right], \\ \{1 /[2-2 \sigma(1-\rho)-\sqrt{ }\{2 \rho \sigma(2-2 \sigma(1-\rho))\}]\}-[\sigma /(1-\sigma)] & \text { for } \rho \in\left((1-\{1 /(2 \sigma)\})^{2}, \sigma / 4\right], \\ 1 & \text { for } \rho \in[\sigma / 4,1 / 2) .\end{cases}
$$

For the no-commitment scenario in the single-generation model, Figure 3B depicts the equilibrium property rights choice in alternative $\{\rho, \sigma\}$ regimes. Figures $3 \mathrm{~A}$ and $3 \mathrm{~B}$ clarify the implications of the credibility of peace commitments (and lack thereof) on property rights choice. On the one hand, the figures show an important similarity between the two scenarios: in both cases, the poorer community 2's optimal choice is distinct from the perfect rights regime when resource inequality is sufficiently large. On the other hand, the figures also reveal an important difference: the poorer community's optimal choice is pure anarchy for a much larger set of $\{\rho, \sigma\}$ values in the commitment scenario as compared to the no-commitment scenario. On reflection, this is just what we should expect. A community will have a weaker incentive to strengthen property rights precisely when value-destroying terminal conflicts can be avoided by credible peace treaties. When property rights are imperfect, the possibility of ex post Coasian bargaining over final consumption dilutes ex ante incentives to strengthen such rights. 
Note that our single-generation model not only generates a divergence in $\pi$-preference between the two communities, it also identifies the poorer community 2 as the one which has the weaker desire to strengthen inherited property rights. This conclusion is indeed dependent on the structure of the guns production function (which we have taken to be of the constant returns-to-scale form). It should not be difficult to understand that if the (common) guns production function exhibited significant increasing returns to scale, then community 1 would have a much greater incentive to militarize than community 2 , and as a result could turn out to be the conflict-prone community with a weaker desire for improving property rights. While this might indeed be the case when there are substantial returns-to-scale in guns production, our next result confirms that for a large class of guns production function (with a limit on the magnitude of returns-to-scale), it is the poorer community that will prefer to devolve to anarchy when resource asymmetry is significant. ${ }^{43}$

Proposition 6: Consider the following guns production function: if community $i$ invests $G_{i}$ in guns, its guns output is $g_{i}=\left(G_{i}\right)^{\gamma}$ for some $\gamma>0$ (while butter production exhibits constant returns to scale). Then for $\sigma \in[1 / 2,1),\left\{R_{1}, R_{2}\right\}$ with $\rho \equiv\left[R_{2} /\left(R_{1}+R_{2}\right)\right] \in(0,1 / 2)$, and $\gamma<1 / \sigma$, community 2 will strictly prefer anarchy to any other rights regime for all $\rho \in\left(0.25, \rho^{-}(\sigma \gamma)\right]$.

Next, we focus on the nature of the rights negotiation game and discuss alternative ways of modeling the need for mutual consent in changing inherited property rights. Suppose that we modeled the rights-negotiation game as a 'split-the-difference arbitration' game, where each community $i$ was required to announce a number $s_{i} \in[0,1]$, and for announcements $\left\{s_{1}, s_{2}\right\}$ the property rights regime $\pi=0.5\left(s_{1}+s_{2}\right)$ would be established. Then it is easy to show that community 1 would first announce $s_{1}=1$ and then the poorer community would strategically underbid in such a way that the following result held: The unique $S P N E$ outcome under split-the-difference arbitration in the single-generation model would lead to the rights regime $\left[\max \left\{1 / 2, \pi^{\dagger}(\rho \mid \sigma)\right\}\right]$. More generally, any arbitration game that sets $\pi=S\left(s_{1}, s_{2}\right)$, where the $S($.) function has the property that $\min \left\{s_{1}, s_{2}\right\} \leq S\left(s_{1}, s_{2}\right) \leq \max \left\{s_{1}, s_{2}\right\}$ with the second inequality being strict whenever $s_{1} \neq s_{2}$, would lead to the non-establishment of perfect property rights in any generation in which $[\pi=1]$ is not the poorer community's optimal choice. Then in the single-generation model, the established rights regime would be strictly weaker than the perfect property rights outcome whenever $\rho<\rho^{+}(\sigma)$.

We conclude this subsection by discussing two other features of our model. For one, we have assumed that within the inherited rights space, it is costless for the communities to alter the regime.

${ }^{43}$ It has been recognized in the extant contest literature that the Hirshleiferian paradox of power might not hold when there are significant increasing returns to scale in guns production; see, for example, Skaperdas and Syropoulos (1997) and Garfinkel and Skaperdas (2000, 2007). In a related context, Sonin (2003) studies a model in which the richer citizens in a community desire weaker property rights due to increasing returns-to-scale in the effectiveness of appropriative weapons. 
Of course, if strengthening property rights is indeed costly, it is likely that our result: "high resource inequality will lead to weakening of the inherited rights regime" will hold with greater force. To understand the issues involved here, note the following. In a generational game where it is costly to implement a property-rights (enforcement) regime $\pi>0$, let $\left\{R_{1}(\pi)>0, R_{2}(\pi)>0\right\}$ denote the endowment vector for the guns-and-butter game after the communities have chosen $\pi \in[0,1]$ (with $\left\{R_{1}(0)>R_{2}(0)>0\right\}$ being the initial endowment vector). We define the resulting aggregate resource and the resulting resource inequality levels respectively as: $R(\pi) \equiv\left[R_{1}(\pi)+R_{2}(\pi)\right]$ and $\rho(\pi)$ $\equiv R_{2}(\pi) / R(\pi)$. Here, it is natural to assume that $R_{i}(\pi)$ will be falling in $\pi$ for $i=1,2$. Then the equilibrium payoffs of the two communities in the guns-and-butter game will have the same structure as the payoffs described in Proposition 2, except that $R($.$) and \rho($.$) will now be functions of$ $\pi$. Then, if initial resource inequality is high with $\rho^{-}(\sigma)>\rho(0)$, fairly general conditions on the rates of change of $R(\pi) \& \rho(\pi)$ will lead to $W_{2}^{\dagger}(\sigma, \pi, R(\pi), \rho(\pi))$ being maximized at $\pi=0$. Specifically, consider the case where $\rho(\pi)$ is non-increasing in $\pi$. In this case, given that $R(\pi)$ is non-increasing in $\pi$, straightforward calculations establish that $W_{2}^{\dagger}(\sigma, \pi, R(\pi), \rho(\pi))$ will be maximized at $\pi=0 .{ }^{44}$

Finally, recognize the following dilemma that can befall the richer community in a particular generation. The richer community can realize that it will be strictly better off if the resource inequality between the two communities can be reduced, even at the cost of a reduction in own resource and an increase in rival resource. To see this, reconsider the static Example II in Section 3.1. Note that if community 1 had voluntarily gifted 50 units of own resource to community 2 at the beginning of their interaction, then, given the post-gift resource vector $\{600,400\}$ (and the inherited rights space $[0,1])$, the communities would have set their rights regime at $\pi=1\left(\right.$ since $0.4>\rho^{+}(1 / 2)=$ 0.375 ) and community 1 would attain utility level 600 (with community 2 attaining utility level 400) and be better-off as compared to the 'no resource gift' equilibrium outcome.

We make two comments regarding the possibility and profitability of such ex ante 'resource gifts', and the concomitant move of the communities towards perfect property rights. Firstly, depending on the nature of the resources, such gifts might be technologically or morally infeasible; e.g., if the

${ }^{44}$ For $i=1,2$, suppose that $R_{i}(\pi) \equiv\left[1-C_{i}(\pi)\right] \times R_{i}(0)$, where $R_{i}(0)$ is community $i$ 's initial resource base and $\left[C_{i}(\pi) \times R_{i}(0)\right]$ is $i$ 's cost of investment in regime $\pi \in[0,1]$. Then assuming $C_{i}(0)=0, C_{i}^{\prime}(\pi) \geq 0$, $C_{i}^{\prime \prime}(\pi) \geq 0$, and $C_{i}(1)<1$ ensures that $R_{i}(\pi)$ will be non-increasing in $\pi$. Then the conditions $\left\{C_{2}{ }^{\prime}(0) \geq\right.$ $C_{1}{ }^{\prime}(0)$ and $C_{2}{ }^{\prime \prime}(\pi) \geq C_{1}{ }^{\prime \prime}(\pi)$ for all $\left.\pi \in[0,1]\right\}$ are together sufficient for $\rho(\pi)$ to be non-increasing in $\pi$. When $\rho(\pi)$ is non-increasing in $\pi$ and $\rho(0)<0.5$, we obtain: $\rho(\pi) \in(0,0.5)$. Then, $W_{2}^{\dagger}(\sigma, \pi, R(\pi), \rho(\pi))$ is immediately obtained from Proposition 2. For fixed $\rho<\rho^{-}(\sigma)$ and $R>0$, we established in Proposition 4 that $W_{2}^{\dagger}(\sigma, \pi, R, \rho)$ strictly decreases in $\pi$. Further, for fixed $\pi<1$ and $R>0$ (resp., for fixed $\pi<1$ and $\rho \in$ $(0,0.5)$ ), it is straightforward to show that $W_{2}^{\dagger}(\sigma, \pi, R, \rho)$ is non-decreasing in $\rho$ (resp., in $R$ ) [for both cases $\rho<\tilde{\rho}(\pi \mid \sigma)$ and $\rho>\tilde{\rho}(\pi \mid \sigma)$ ]. Then, since $\rho(0)<\rho^{-}(\sigma)$, and $R(\pi)$ and $\rho(\pi)$ are taken to be non-increasing in $\pi$, so $W_{2}^{\dagger}(\sigma, \pi, R(\pi), \rho(\pi))$ is strictly decreasing in $\pi$ and hence it is maximized at $\pi=0$. 
resources under consideration are indeed aggregate human capital of a community, such resource transfers would require gifting of people (involving a change of community identity / citizenship for these people). ${ }^{45}$ Secondly, the existence of such profitable resource transfers (if feasible) will depend crucially on the extent of institutional inertia present in the system. Recognize that if in the example discussed in the previous paragraph, the inherited rights space was $[0,0.6]$ (e.g., with $\pi(0)=\varepsilon=\eta=$ 0.3 ) then no profitable resource transfer would exist for community 1 . This is so because after any or no resource transfer from 1 to 2 , the collectively set rights regime would be $\pi=0.6$ (since $W_{2}^{\dagger}($.) would be maximized at the upper boundary of the inherited rights space), and given that it would be optimal for community 1 to offer no resource gift. ${ }^{46}$

\section{Equilibrium Dynamics in the Sequence- of-Generations Model}

In analyzing the sequence-of-generations model, we take as given the feasible structural parameters: the value-survival parameter $\sigma \in[1 / 2,1)$, the institutional-inertia parameters $\{\varepsilon, \eta\} \in(0,1] \times(0,1]$, and the resource law-of motion (RLM) function $F($.) that is assumed to be 'well-behaved' (as defined in Section 2.2). Given these parameters, our dynamic model starts with the following feasible initial conditions: a rights space $\Pi(1)$ that is jointly inherited by first generation of residents in the two communities, with $\Pi(1) \equiv[\pi(0)-\varepsilon, \pi(0)+\eta] \cap[0,1]$ for some $\pi(0) \in[0,1](\pi(0)$ can be taken to be the rights regime set by the predecessors of the first generation); and community-specific resource endowments $\left\{R_{1}(1), R_{2}(1)\right\}$ satisfying $R(1) \equiv\left[R_{1}(1)+R_{2}(1)\right]>0$ and $\rho(1) \equiv\left\{R_{2}(1) / R(1)\right\} \in(0,1 / 2)$. In the first $(t=1)$ generation, the communities establish their generational property rights regime $\pi^{*}(1) \in \Pi(1)$ via the rights negotiation game. ${ }^{47}$ Then given $\left\{R_{1}(1), R_{2}(1) ; \pi^{*}(1)\right\}$, the residents of the two communities play their guns-and-butter game. This game generates equilibrium butter production and consumption vectors $\left\{B_{1} *(1), B_{2} *(1)\right\}$ and $\left\{W_{1}^{*}(1), W_{2}^{*}(1)\right\}$. The first-generation outcomes lead to the inheritances for the second $(t=2)$ generation: $R_{i}^{*}(2) \equiv F\left(R_{i}(1), B_{i}^{*}(1), W_{i}^{*}(1)\right)$ for $i=1,2$, and $\Pi^{*}(2) \equiv\left[\pi^{*}(1)-\varepsilon, \pi^{*}(1)+\eta\right] \cap[0,1]$. In equilibrium, $\left\{R_{1} *(2), R_{2} *(2)\right\}$ will satisfy: $R^{*}(2) \equiv\left[R_{1}{ }^{*}(2)+R_{2} *(2)\right]>0$ and $\rho^{*}(2) \equiv\left\{R_{2}{ }^{*}(2) / R^{*}(2)\right\} \in(0,1 / 2)$; see Lemma 7 below. Then the

\footnotetext{
45 Apart from the outright transfer of resources, community 1 could also contemplate a commitment to selfdestruction of a part of its own resource (or a withdrawal of a part of its resources from productive activities). But such a commitment will not be credible because after the rights regime is strengthened, community 1 will have the incentive to renege and employ all of its resources in the production processes.

${ }^{46}$ If it is indeed that case that resource-gifts are feasible, and there is no institutional inertia, the reader can consider our analysis to be presenting a reasonable scenario in which 'foreign aid' can arise exclusively out of self-interest (where such aid leads to a Pareto-improving improvement in property rights).

47 This game is analogous to the one described for the single-generation model in Section 4.2, except that $\Pi(1)$ is not necessarily the full interval $[0,1]$. Consequently, the negotiations will establish that rights regime which is optimal in $\Pi(1)$ for the poorer community 2.
} 
second generation residents of the two communities make their decisions in the same sequence as their predecessors, and the process repeats itself generation after generation.

Our next result establishes two facts: equilibrium trajectories of chosen property rights $\left\{\pi^{*}(t)\right\}_{t=1}^{\infty}$ and of realized resource inequality $\left\{\rho^{*}(t)\right\}_{t=1}^{\infty}$ are unique, and the resource-ranking of the two communities is preserved in the dynamic equilibrium of the sequence-of-generations model.

LEMMA 7: Given feasible structural parameters and initial conditions, if the communities inherit $\left\{R_{1} *(t), R_{2}^{*}(t) ; \Pi^{*}(t)\right\}$ in generation $t$ with $R^{*}(t) \equiv\left[R_{1} *(t)+R_{2} *(t)\right]>0$ and $\rho^{*}(t) \equiv\left\{R_{2} *(t) / R^{*}(t)\right\} \in(0$, $1 / 2)$, and $\Pi^{*}(t)=\left[\pi^{*}(t-1)-\varepsilon, \pi^{*}(t-1)+\eta\right] \cap[0,1]$, then, in generation $t$ we have for $i=1,2: \pi^{*}(t)=$ $\max \left[\pi^{*}(t-1)-\varepsilon, \min \left\{\pi^{\dagger}\left(\rho^{*}(t) \mid \sigma\right), \pi^{*}(t-1)+\eta\right\}\right] ; B_{i}^{*}(t)=B_{i}^{\dagger}\left(\sigma, \pi^{*}(t), R^{*}(t), \rho^{*}(t)\right)$; and $W_{i}^{*}(t)=$ $W_{i}^{\dagger}\left(\sigma, \pi^{*}(t), R^{*}(t), \rho^{*}(t)\right)$. Then generation $t+1$ 's inheritance vector $\left\{R_{1}^{*}(t+1), R_{2}^{*}(t+1) ; \Pi^{*}(t+1)\right\}$ is unique with $\Pi^{*}(t+1)$ a compact subset of $[0,1]$ and $\rho^{*}(t+1) \equiv\left\{R_{2}^{*}(t+1) / R^{*}(t+1)\right\} \in(0,1 / 2) .{ }^{48}$

Given the existence of a unique equilibrium path $\left\{\pi^{*}(t), \rho^{*}(t)\right\}_{t=1}^{\infty}$ in our sequence-of-generations model, we turn our attention to uncovering the robust properties of this path starting from alternative feasible configurations of our structural parameters and initial conditions.

\subsection{Evolution of Property Rights: General Results}

Recognize the following interplay between inherited resource asymmetry and the chosen property rights across generations. In every generation $t$, the more equal (resp., unequal) is the resource distribution, the greater will be the collective desire to strengthen (resp., weaken) the rights regime. A stronger (resp., weaker) rights regime will then cause the generational butter-production ratio $\left(B_{1} *(t) / B_{2} *(t)\right)$ to be lower (resp., higher) and the butter-consumption ratio $\left(W_{1} *(t) / W_{2} *(t)\right)$ to be higher (resp., lower). Eventually the resource law-of-motion will map these outcome-ratios to the resource distribution of the following generation $t+1$.

In order to identify the long-run implications of this dynamic interplay between resource distribution and property rights, we define the following possible long-run features of the timetrajectories of the chosen rights regime and the realized resource inequality:

- An equilibrium path $\left\{\pi^{*}(t), \rho^{*}(t)\right\}_{t=1}^{\infty}$ will be said to 'devolve to perpetual anarchy' in the longrun if and only if there exists $t^{\mathrm{A}} \geq 1$ such that $\pi^{*}(t)=0$ for all $t \geq t^{\mathrm{A}}$.

- $\left\{\pi^{*}(t), \rho^{*}(t)\right\}_{t=1}^{\infty}$ will be said to 'attain perfect property rights' in the long-run if and only if for every $\psi>0$ (however small) there exists $t^{\mathrm{P}}(\psi) \geq 1$ such that $\pi^{*}(t) \geq[1-\psi]$ for all $t \geq t^{\mathrm{P}}(\psi)$.

${ }^{48}$ In our dynamic model, the exogenous variables are: $\sigma,\{\varepsilon, \eta\}, F(),. \Pi(1), R(1) \equiv R_{1}(1)+R_{2}(1)$ and $\rho(1) \equiv$ $R_{2}(1) / R(1)$. All other variables are endogenous in that they are determined directly by current community actions or indirectly by past actions; endogenous variables are consistently marked by $\{*\}$ 's in our analysis. This is to be kept in mind while reading Lemma 7 as its statement does abuse notation for the first generation. 
- $\left\{\pi^{*}(t), \rho^{*}(t)\right\}_{t=1}^{\infty}$ will be said to 'lead to high resource inequality' in the long-run if and only if there exists $t^{\mathrm{H}} \geq 1$ such that $\rho^{*}(t) \leq \rho^{-}(\sigma)$ for all $t \geq t^{\mathrm{H}}$.

- $\left\{\pi^{*}(t), \rho^{*}(t)\right\}_{t=1}^{\infty}$ will be said to 'lead to low resource inequality' in the long run if and only if for every $\xi>0$ (however small) there exists $t^{\mathrm{L}}(\xi) \geq 1$ such that $\rho^{*}(t) \geq\left[\rho^{+}(\sigma)-\xi\right]$ for all $t \geq t$ $\mathrm{L}(\xi) .{ }^{49}$

With these definitions in hand, we identify a central feature of the dynamic equilibrium in our sequence-of-generations model: in the long run, the property rights path $\left\{\pi^{*}(t)\right\}_{t=1}^{\infty}$ and the resource inequality path $\left\{\rho^{*}(t)\right\}_{t=1}^{\infty}$ have the following interconnections.

PROPOSITION 8: Given feasible structural parameters and initial conditions, the equilibrium path $\left\{\pi^{*}(t), \rho^{*}(\mathrm{t})\right\}_{\models 1}^{\infty}$ devolves to perpetual anarchy if and only if it leads to high resource inequality, while the path attains perfect property rights if and only if it leads to low resource inequality.

Proposition 8 clarifies that the forces that cause resource inequality to eventually and permanently cross a high threshold (ensuring $\rho^{*}(t) \leq \rho^{-}(\sigma)$ for high values of $t$ ) also induce the communities to eventually and permanently reside in anarchy. On the other hand, the forces that lead to persistent low resource inequality in the long-run (ensuring $\rho^{*}(t)$ is greater than or arbitrarily close to $\rho^{+}(\sigma)$ for high values of $t$ ) also cause the communities to eventually attain $\pi=1$ (or $\pi$ arbitrarily close to 1 ) in the long-run. In what follows, we prove that the magnitude of the initial resource inequality $\rho(1)$, and the structure of the $R L M$ - especially, the relative dependence of a community's resources on its past butter-investment and butter-consumption - are important variables that determine the nature of the interconnected long-run trajectories of resource inequality and property rights.

We begin by considering the 'autonomous' $R L M$ structure in which, for each community $i$, the $R L M$

${ }^{49}$ We define a time-path that attains perfect property rights, and a time-path that attains low resource inequality, in this complicated manner for the following reason. Numerical simulations show that for certain structural parameters and initial conditions, the equilibrium time-path gets arbitrarily close to $\pi=1$ while never attaining it, and concurrently, $\rho^{*}(t)$ gets arbitrarily close to the critical value of $\rho^{+}(\sigma)$ while never attaining or exceeding it; while for other structural parameters and initial conditions, the equilibrium time-path exactly attains $\pi=1$ in finite time, and $\rho^{*}(t)$ exactly attains or exceeds $\rho^{+}(\sigma)$ in finite time. To understand this, consider the case where there is no institutional inertia. Then as resource inequality falls over time, property rights are set exactly equal to the myopically optimal rights choice of the poorer community in each generation. As $\pi$ approaches unity in step with the decrease in resource inequality, the extent of further decrease in resource inequality would become smaller and smaller. Under some parametric conditions, it is possible for resource inequality to converge to a value such that the myopically optimal rights choice of the poorer community is always strictly less than 1, though it will become arbitrarily close to 1 (recall the dynamic examples in Section 3.2). In our analytical results, we do not distinguish between these two kinds of time-paths and simply group them together as one larger class of time-paths possessing the common feature that the equilibrium rights regime becomes arbitrarily close to perfect in the long-run. 
function has only $R_{i}$ as its argument. For this class of $R L M \mathrm{~s}$, we have the following result:

PROPOSITION 9: Given feasible structural parameters and initial conditions, suppose that the $R L M$ has the following autonomous structure: $R_{i}(t+1)=h \cdot\left[R_{i}(t)\right]^{m}$, with $h>0, m>0$ for $i=1,2$ and $t \geq 1$.

[A] If $m>1$ then the equilibrium path $\left\{\pi^{*}(t), \rho^{*}(\mathrm{t})\right\}_{t=1}^{\infty}$ will devolve to perpetual anarchy.

[B] If $m<1$ then the equilibrium path $\left\{\pi^{*}(t), \rho^{*}(\mathrm{t})\right\}_{=1}^{\infty}$ will attain perfect property rights.

[C] If $m=1$ then the equilibrium path $\left\{\pi^{*}(t), \rho^{*}(\mathrm{t})\right\}_{t=1}^{\infty}$ will devolve to perpetual anarchy if and only if $\rho(1) \leq \rho^{-}(\sigma)$, while it will attain perfect property rights if and only if $\rho(1) \geq \rho^{+}(\sigma) .{ }^{50}$

When the RLM is autonomous, the behavior of past generations of community residents does not influence current resource distribution. Then a strictly convex autonomous $R L M$ forces resource inequality to increase monotonically over time, and that causes the chosen rights regime to devolve to perpetual anarchy. The opposite happens when the autonomous $R L M$ is strictly concave. In contrast, a linear $R L M$ generates an unchanging level of resource inequality, and then the 'static preferences' of the poorer community 2 (as described in Proposition 4) determine the long-run resting point of the rights regime: a small initial inequality for which $\pi^{\dagger}(\rho \mid \sigma)=1$ leads to continual strengthening of property rights while a large initial inequality for which $\pi^{\dagger}(\rho \mid \sigma)=0$ leads to anarchy.

Our next proposition states the most general relationship that we can uncover between initial resource inequality, the structure of the $R L M$, and the nature of evolution of property rights. Here we restrict attention to 'homogeneous' $R L M \mathrm{~s}-$ i.e., $R L M \mathrm{~s}$ that satisfy the following homogeneity condition: for all $R_{i}>0, B_{i} \geq 0, W_{i} \geq 0, k>0, F\left(k \cdot R_{i}, k \cdot B_{i}, k \cdot W_{i}\right)=k^{n} . F\left(R_{i}, B_{i}, W_{i}\right)$ for some $n>0$. Next, we define three alternative properties of the $R L M$ as follows. For $i=1,2$, and for any $\sigma \in[1 / 2,1), \pi \in[0,1], R>0$, and $\rho \in(0,1 / 2)$, recall the definitions of $B_{i}^{\dagger}(\sigma, \pi, R, \rho)$ and $W_{i}^{\dagger}(\sigma, \pi, R, \rho)$ from Proposition 2, and define the following ratio:

$$
r^{\dagger}(\sigma, \pi, R, \rho) \equiv\left\{F\left(\rho R, B_{2}^{\dagger}(.), W_{2}^{\dagger}(.)\right) /\left[F\left(\rho R, B_{2}^{\dagger}(.), W_{2}^{\dagger}(.)\right)+F\left((1-\rho) R, B_{1}^{\dagger}(.), W_{1}^{\dagger}(.)\right)\right]\right\} .
$$

Note that $r^{\dagger}($.) gives the 'equilibrium resource-inequality evolution' between two generations: if $\{R(t)>0, \rho(t) \in(0,1 / 2), \pi(t) \in[0,1)\}$ are the inherited resource base, resource inequality, and the chosen rights regime in generation $t$, then equilibrium resource inequality inherited by generation $(t+1)$ will be: $\rho^{*}(t+1)=r^{\dagger}(\sigma, \pi(t), R(\mathrm{t}), \rho(t))$. Further, given $\sigma \in[1 / 2,1), \pi \in[0,1), R>0$, and $\rho \in(0,1 / 2)$ we define: Property F-1.1: For all $\pi \in[0,1), r^{\dagger}(\sigma, \pi, R, \rho)<\max \{\rho, \tilde{\rho}(\pi \mid \sigma)\}$.

Property F-1.2: Property F-1.1 and in addition: $r^{\dagger}(\sigma, \pi=1, R, \rho)<\rho$ whenever $\rho \geq \rho^{+}(\sigma)$. Property F-2: For any $\pi \in[0,1)$, if $\rho \geq \tilde{\rho}(\pi \mid \sigma)$ then $r^{\dagger}(\sigma, \pi, R, \rho)>\rho$, and if $\rho<\tilde{\rho}(\pi=0 \mid \sigma)$ then $r^{\dagger}(\sigma$,

${ }^{50}$ When $m=1$ and $\rho(1) \in\left(\rho^{-}(\sigma), \rho^{+}(\sigma)\right)$, then $\left\{\pi^{*}(t), \rho^{*}(t)\right\}_{t=1}{ }^{\infty}$ will exhibit 'perpetually intermediate property rights': there exists $0<\pi^{-}<\pi^{+}<1$ such that $\pi^{*}(t) \in\left(\pi^{-}, \pi^{+}\right)$for all $t \geq 1$. 
$\pi=0, R, \rho)>\rho$; in addition, $r^{\dagger}(\sigma, \pi=1, R, \rho) \geq \rho$ whenever $\rho \geq \rho^{+}(\sigma)$.

Properties F-1.1 and F-1.2 contain alternative requirements for the equilibrium inherited resource inequality between the communities to increase over generations, while Property F-2 requires just the opposite to happen. As we show below, many RLM specifications will satisfy these properties.

Proposition 10: Take as given the set of feasible structural parameters and initial conditions. [A] The equilibrium path $\left\{\pi^{*}(t), \rho^{*}(t)\right\}_{t=1}^{\infty}$ will devolve to perpetual anarchy in either one of the following two scenarios: (i) $F($.) is homogeneous and satisfies Property F-1.1, and it is the case that $\rho(1)<\rho^{+}(\sigma)$; and (ii) $F($.) is homogeneous and satisfies Property F-1.2.

[B] The equilibrium path $\left\{\pi^{*}(t), \rho^{*}(\mathrm{t})\right\}_{t=1}^{\infty}$ will attain perfect property rights if $F($.$) is homogeneous$ and satisfies Property F-2.

Given increasing resource inequality, Proposition 10 states that the chosen property rights will devolve to anarchy, especially when the initial resource inequality is also significant. In contrast, if the resource inequality lessens over time then the communities will collectively move towards establishing perfect property rights. This is a central result of our analysis:

Failure to collectively strengthen the property rights regime over time is an equilibrium possibility. A prime cause of this is persistently high inequality in resource endowments across communities due to significant initial inequality and/or exacerbation of resource inequality over generations.

Further, the following points bear emphasis. Firstly, note that Properties F-1 and F-2 assume nothing about the magnitude of change in resource inequality between successive generations; they can thus be satisfied by a large class of resource laws-of-motion, and for each member of this class Proposition 10 establishes that (given certain initial conditions) the chosen rights regime converges in the long-run to one extreme (anarchy) or the other (perfect property rights). ${ }^{51}$ Secondly, note that Propositions 9 and 10 hold for all extents of institutional inertia; thus, for a large number of cases the institutional constraints will not affect the long-run position of the property rights regime (they only affect the speed at which the position is attained).

In the next subsection, we study a special class of the resource law-of-motion - the 'linear homogeneous' RLM. Focusing on this class of RLMs allows us to identify the factors that can cause exacerbation of resource inequality over time (and thus connect our formal analysis to the dynamic examples presented in Section 3).

\subsection{Evolution of Property Rights: The Linear Homogeneous Model}

${ }^{51}$ In the following section, we explore the ways in which the conclusions of Proposition 10 hold in the case of linear-homogeneous $R L M \mathrm{~s}$. Footnote 53 clarifies that our results also hold for alternative $R L M$ structures that satisfy either Property F-1 or Property F-2. 
In this subsection, we focus on the following (well-behaved) linear homogeneous $R L M$ :

$$
F\left(R_{i}, B_{i}, W_{i}\right)=\left[1+\left\{\mu+v\left[\lambda\left(B_{i} / R_{i}\right)+(1-\lambda)\left(W_{i} / R_{i}\right)\right]\right\}\right] R_{i} \text {, with } \mu \geq 0, v>0 \text {, and } \lambda \in(0,1) .
$$

Under this specification, the per-generation (history-dependent) growth rate in each community $i$ 's resources will be: $\left.\left\{\mu+v \lambda \cdot\left[B_{i}^{*}(t) / R_{i}^{*}(t)\right]+v(1-\lambda) \cdot\left[W_{i}^{*}(t) / R_{i}^{*}(t)\right)\right]\right\}$, while the per-generation aggregate resource growth rate will be: $\left\{\mu+v \cdot\left[\left(B_{1} *(t)+B_{2} *(t)\right) / R^{*}(t)\right]\right\} .{ }^{52}$ Note that since $\left[B_{1} *(t)+\right.$ $\left.B_{2} *(t)\right]$ will equal $\left[W_{1}^{*}(t)+W_{2} *(t)\right]$ in every generational equilibrium, the aggregate resource growth rate will not be immediately influenced by $\lambda$. But $\lambda$ will affect each community's resource growth and therefore the evolution of resource inequality; this in turn will affect each community's future resource growth rate and therefore future (and long-term) aggregate resource growth. In what follows, we delineate how different magnitudes of the coefficients vector $\{\mu, \nu, \lambda\}$ affect the evolution of resource inequality over generations and thus the time-trajectory of the chosen property rights regimes. (Note that the case ' $v=0$ ' has been studied in Proposition $9[\mathrm{C}]$.) Recall our dynamic examples in Section 3.2, where we showed that in the case of the linear homogeneous $R L M$, the identity of the relatively important growth-driver - past creative investment or past consumption - turns out to be an important determinant of time-trajectory of property rights. Our next proposition states the formal result in this regard.

Proposition 11: Take as given the set of feasible structural parameters and initial conditions, and assume that the $R L M$ is of the linear homogeneous form.

[A] If $\lambda \in([1-0.5 \sigma], 1)$, then the $R L M$ satisfies Property F-1.1, and so the equilibrium path $\left\{\pi^{*}(t), \rho^{*}(t)\right\}_{t=1}^{\infty}$ devolves to perpetual anarchy if $\rho(1)<\rho^{+}(\sigma)$.

[B] If $\lambda \in(0,[1 /(1+\sigma)])$, then the $R L M$ satisfies Property $\mathrm{F}-2$, and so the equilibrium path $\left\{\pi^{*}(t)\right.$, $\left.\rho^{*}(t)\right\}_{t=1}^{\infty}$ attains perfect property rights.

The intuition behind Proposition 11 is the following. In our sequence-of-generations model, the poorer community 2 is the conflict-prone bribe-taker in every generation, implying that $\left[B_{1} *(t)-\right.$ $\left.W_{1} *(t)\right] \geq 0 \geq\left[B_{2} *(t)-W_{2}^{*}(t)\right]$. Further, for all $t \geq 1$ we have: $W_{2}^{*}(t) / W_{1} *(t) \geq R_{2}(t) / R_{1}(t) \geq$ $B_{2} *(t) / B_{1} *(t)$, with all four inequalities being strict when $\pi^{*}(t)<1$. As a result, when resource growth in each community follows the linear homogeneous $R L M$, a high value of $\lambda$ causes past butter production (i.e., skill acquisition) to be the more important driver of resource growth. That generates higher growth in $R_{1}$ vis-à-vis $R_{2}$. When this process starts from an initial situation of large resource inequality $\left(\rho(1)<\rho^{+}(\sigma)\right)$, then the monotonically increasing resource inequality is

${ }^{52}$ Here, the maximal rate of aggregate resource growth can be $(\mu+v)$; it will be reached in a generation if and only if both communities use all their resources for value creation. Also note the following: The linearhomogenous RLM permits us to study the (relative) effects of resource growth led by past skill-acquisition $v s$. past nutrition in a clean way, by ensuring that the cross-effects of these growth drivers are zero. 
accompanied by devolution to perpetual anarchy. In contrast, a low value of $\lambda$ causes past butter consumption (i.e., nutrition) to be the more important driver of resource growth. That leads to higher growth in $R_{2} v i s$ - $\grave{a}$-vis $R_{1}$, and that decreases resource inequality across generations. The intertemporal equalization of resource distribution goes hand in hand with attaining perfect property rights in the long-run. Proposition 11 identifies the threshold values of $\lambda$ and $\rho(1)-{ }^{~ ' h i g h ~} \lambda$ and not too high $\rho(1)^{\prime} v s$. 'low $\lambda$ ' - for these opposite time-trajectories to arise in equilibrium. ${ }^{53}$

Note that these $\{\lambda, \rho(1)\}$ thresholds depend only on the value-survival parameter $\sigma$, and not on the institutional inertia parameters. ${ }^{54}$ So when $\{\lambda, \rho(1)\}$ satisfy specific threshold conditions, the extent of institutional inertia does not influence the long-run values of resource inequality and property rights, but only their 'transition trajectories' from their initial values to their long-run values. In this context, it is worthwhile to review the dynamic examples studied in Sections 3.2.1 and 3.2.2. In those examples, $R L M$ structure [I] with $\lambda=0.4$ satisfies the hypothesis of Proposition 11[B], while $R L M$ structure [II] with $\lambda=0.8$ satisfies the hypothesis of Proposition 11[A]. Consequently, irrespective of institutional inertia, [I] leads to perfect property rights while [II] leads to perpetual anarchy in the long-run. But the short-run trajectories of $\pi(t)$ do depend on the extent of

53 Different extensions of Proposition 11, for 'non-linear', 'non-separable', and 'time-varying' linear RLMs, hold in our model. Below we indicate a few of these results; the proofs are available from the authors. First, consider the following non-linear generalization of the linear homogeneous $R L M: F\left(R_{i}, B_{i}, W_{i}\right)=$ $\left[x .\left(R_{i}\right)^{\chi}+y \cdot\left(B_{i}\right)^{\chi}+z \cdot\left(W_{i}\right)^{\chi}\right]$ with $\chi>0, x \geq 1, y>0, z>0$. In the case where $\{y / z>(2-\sigma) / \sigma\}$, for $\chi>1$ the $R L M$ satisfies Property F-1.2, and so the equilibrium path $\left\{\pi^{*}(t), \rho^{*}(t)\right\}_{t=1}^{\infty}$ devolves to anarchy for all $\rho(1)$ $\in(0,0.5)$. Alternatively, in the case where $\{y / z<1 / \sigma\}$, for $\chi<1$ the $R L M$ satisfies Property F-2, and so the equilibrium path $\left\{\pi^{*}(t), \rho^{*}(t)\right\}_{t=1}^{\infty}$ attains perfect property rights for all $\rho(1) \in(0,0.5)$.

Next, consider the following non-separable $R L M \mathrm{~s}$. If the $R L M$ has the structure: $F\left(R_{i}, B_{i}, W_{i}\right)=\left[x \cdot R_{i}\left(\chi_{1}+\chi_{2}\right)\right.$ $\left.+y \cdot R_{i}{ }^{\chi_{1}} \cdot B_{i}{ }^{\chi_{2}}\right]$ with $\left\{x \geq 1, y>0, \chi_{1}>0, \chi_{2}>0\right\}$ and $\left[\chi_{1}+\chi_{2}\right]>1$ then the homogeneous $R L M$ satisfies Property F-1.2, and so the equilibrium path $\left\{\pi^{*}(t), \rho^{*}(t)\right\}_{t=1}^{\infty}$ devolves to anarchy for all $\rho(1) \in(0,0.5)$. Alternatively, if the $R L M$ has the structure: $F\left(R_{i}, B_{i}, W_{i}\right)=\left[x \cdot R_{i}{ }^{\left(\chi_{1}+\chi_{3}\right)}+z \cdot R_{i}{ }^{\chi_{1}} \cdot W_{i}{ }^{\chi_{3}}\right]$ with $\left\{x \geq 1, z>0, \chi_{1}>\right.$ $\left.0, \chi_{3}>0\right\}$ and $\left[\chi_{1}+\chi_{3}\right] \leq 1$ then the homogeneous $R L M$ satisfies Property F-2, and so the equilibrium path $\left\{\pi^{*}(t), \rho^{*}(t)\right\}_{t=1}^{\infty}$ attains perfect property rights for all $\rho(1) \in(0,0.5)$.

Finally, consider the following time-varying linear $R L M: F\left(R_{i}, B_{i}, W_{i} ;(\rho, \pi)\right)=x(\rho, \pi) \cdot R_{i}+y(\rho, \pi) \cdot B_{i}+z(\rho$, $\pi) . W_{i}$, where $x() \geq 1,. y()>$.0 and $z()>$.0 are continuous functions of their arguments (and where $\rho \in(0,0.5]$ and $\pi \in[0,1])$. If $\{y(\rho, \pi) / z(\rho, \pi)>(2-\sigma) / \sigma\}$ for all $\rho$ and $\pi$, then the equilibrium path $\left\{\pi^{*}(t), \rho^{*}(t)\right\}_{t=1}^{\infty}$ devolves to anarchy for all $\rho(1) \in\left(0, \rho^{+}(\sigma)\right)$. Alternatively, if $\{y(\rho, \pi) / z(\rho, \pi)<1 / \sigma\}$ for all $\rho$ and $\pi$, then the equilibrium path $\left\{\pi^{*}(t), \rho^{*}(t)\right\}_{t=1}^{\infty}$ attains perfect property rights for all $\rho(1) \in(0,0.5)$.

${ }^{54}$ Note that both the $\lambda$-thresholds increase as $\sigma$ decreases, implying that as the cost of conflict increases there will be a wider range of $\lambda$ values for which perfect property rights are attained. Recognize that in a single generation, if the cost of conflict is higher (i.e., $\sigma$ is lower) then the poorer community will be likelier to choose lower property rights (since $\rho^{+}(\sigma)$ and $\rho^{-}(\sigma)$ are both decreasing in $\sigma$ ), but in the long-run there will be a greater likelihood for the attainment of perfect property rights. 
institutional inertia. In our example, not only is the speed of convergence of $\pi(t)$ to its long-run value determined by the magnitudes of $\{\varepsilon, \eta\}$, these parameters also determine the monotonicity property of $\pi(t)$ and the nature of its convergence to the long-term value.

Next, we explore another possible impact of institutional inertia in our sequence-of-generations model. We focus on situations in which the parameters $\{\lambda, \rho(1)\}$ do not satisfy the threshold conditions stated in Proposition 11, and demonstrate that in such situations, the existence of specific kinds of institutional inertia might force the dynamic equilibrium into anarchy in the long-run. Specifically, in the parameter configurations examined in the following proposition, institutional inertia turns out to be an important causal factor for devolution to perpetual anarchy.

PROPOSITION 12: Take as given the set of feasible structural parameters and initial conditions, and assume that the $R L M$ is of the linear homogeneous form.

[A] In the case where $\lambda \in([1-0.5 \sigma], 1)$ and $\rho(1)>\rho^{+}(\sigma)$, given any $\pi(0) \in[0,1)$ there exists $\eta^{0} \in$ $(0,1)$ such that if $\eta<\eta^{0}$, the equilibrium path $\left\{\pi^{*}(t), \rho^{*}(t)\right\}_{t=1}^{\infty}$ devolves to anarchy.

[B] Alternatively, in the case where $\lambda \in([1 /(1+\sigma)],[1-0.5 \sigma])$, given any $\pi(0) \in[0,1)$ there exists $\rho^{0}(\sigma) \in\left(\rho^{-}(\sigma), \rho^{+}(\sigma)\right)$ and $\varepsilon^{0} \in(0,1)$ such that if $\rho(1)<\rho^{0}(\sigma)$ and $\varepsilon>\varepsilon^{0}$, the equilibrium path $\left\{\pi^{*}(t), \rho^{*}(\mathrm{t})\right\}_{t=1}^{\infty}$ devolves to anarchy.

It is worthwhile to spell out the import of the two parts of Proposition 12. First, consider the case where $\lambda$ satisfies its threshold condition $\{\lambda>[1-0.5 \sigma]\}$ but $\rho(1)$ does not (so we have: $(\rho(1)>$ $\left.\rho^{+}(\sigma)\right)$. In this case, sufficient resource equality will encourage the first generation to establish perfect property rights, and if institutional inertia does not block this process, then the perfect rights regime will be established for all times. But if institutional inertia permits only incremental strengthening of $\pi(t)$ in every period, then the 'high $\lambda$ ' might cause an inter-generational increase in resource inequality to a point at which the poorer community 2 starts preferring a weakening of the rights regime (i.e., for $\rho(t)$ to fall over time below $\rho^{+}(\sigma)$ ). If that is the case, then the property rights trajectory will eventually reverse itself permanently and fall continually to zero. This is the possibility that Proposition 12[A] formalizes.

Next, consider the case where $\lambda$ does not satisfy its threshold condition $\{\lambda \in([1 /(1+\sigma)],[1-0.5 \sigma])\}$ while $\rho(1)$ is not too large $\left\{\rho(1)<\rho^{0}(\sigma)\right.$ with $\left.\rho^{0}(\sigma) \in\left(\rho^{-}(\sigma), \rho^{+}(\sigma)\right)\right\} .^{55}$ In this case where the $R L M$ has an 'intermediate $\lambda$ ', whenever $\rho$ is low (i.e., $\rho<\rho^{0}(\sigma)$ ) and $\pi$ is high (i.e., $\pi>\pi^{\dagger}\left(\rho^{0}(\sigma) \mid \sigma\right)$ ) they

55 Here, the relative importance of past butter production vis-à-vis butter consumption as the growth-driver is such that: if $\pi(0)$ is high and there is sufficiently high institutional inertia, then the sequence of generational equilibria might cause resource inequality to fall over generations to a sufficiently low level, causing the residents to eventually collectively push up $\pi(t)$ towards one; in contrast, when there is little or no institutional inertia then there will be devolution of $\pi(t)$ to anarchy. 
will move in opposite directions $-\pi$ will decrease towards the myopically optimal regime for the poorer community, while high $\pi$ will cause inter-generational reduction in resource inequality (i.e., a rise in $\rho$ ). Effectively, there will be a 'race' between $\pi$ and $\rho$, with $\pi$ moving down towards $\pi^{\dagger}\left(\rho^{0}(\sigma) \mid \sigma\right)$ and $\rho$ moving up towards $\rho^{0}(\sigma)$. Eventually, both will end up on the same side of their respective critical points. The presence of high institutional inertia, which will slow down the movement in $\pi$, will likely cause $\rho$ to win the race, and for $\rho>\rho^{0}(\sigma)$ and $\pi>\pi^{\dagger}\left(\rho^{0}(\sigma) \mid \sigma\right)$ to eventually obtain. Then, the property rights trajectory will reverse itself permanently and perfect property rights will be attained. In contrast, sufficiently low or no institutional inertia will cause $\pi$ to win the race and for $\rho<\rho^{0}(\sigma)$ and $\pi<\pi^{\dagger}\left(\rho^{0}(\sigma) \mid \sigma\right)$ to eventually obtain. Then, low property rights will exacerbate resource inequality over time, and so the resource inequality trajectory will reverse itself and there will be eventual attainment of high resource inequality and corresponding devolution of property rights to anarchy. Proposition 12[B] formalizes this possibility.

Under a linear-homogeneous $R L M$, what happens to the 'size' of the poorer community in the longrun when the system devolves to perpetual anarchy? We had asked this question in Section 3.2.1 and had found that in our example, community 2 became a pure bandit at some point but survived with an eventually-stable resource share. Our next proposition proves the robustness of this result.

Proposition 13: Assume that the RLM is of the linear homogeneous form. If the feasible structural parameters and the initial conditions are such that the equilibrium path devolves to anarchy, then, whenever $\lambda \neq 1 /[1+\sigma], \rho^{*}(t)$ converges to a constant $\rho^{\infty} \in(0,[0.5 \sigma /(1+\sigma)])$ and there exists $\tau>0$ such that community 2 is a bandit in all generations $t>\tau .{ }^{56}$

Having presented characterization results for the equilibrium evolution of property rights, we now turn to the issue of evolution of community welfare in our sequence-of-generations model.

\subsection{Evolution of Community Welfare}

Restricting attention to the case of linear-homogenous $R L M$ s studied in Section 5.2, we first ask the following question: Is a scenario where two communities work together to attain perfect property rights necessarily associated with higher intertemporal community welfare as compared to a scenario where property rights get continually weakened to anarchy? In response, we argue as follows: While the intuition that improving property rights is associated with better welfare outcomes is certainly valid in the long-run, that need not be the case in the short run.

\footnotetext{
${ }^{56}$ In the non-generic case where $\lambda=1 /[1+\sigma]$, then the equilibrium path can devolve to anarchy, but if it does then it will converge to some $r^{\infty} \in\left[0.5 \sigma /[1+\sigma], \rho^{-}(\sigma)\right]$. In contrast, given a (possibly non-linear) $R L M$ that does not depend on past consumption (e.g., an $R L M$ of the form: $F\left(R_{i}, B_{i}, W_{i}\right)=x .\left(R_{i}\right)^{\chi}+y .\left(B_{i}\right)^{\chi}$ with $\chi \geq$ $1, x \geq 1, y>0)$, it is straightforward to show that $\rho^{*}(t)$ can converge to zero in the long-run.
} 
We establish our result by considering the following scenario: There exist two different 'economies' E1 and E2, each of which is made up of the two-community structure that we study in this paper. Suppose that the structural parameters (and initial conditions) of the two economies differ in a way such that, starting from a common initial date $t=1$, economy E1 attains perfect property rights in the long-run while economy E2 devolves to perpetual anarchy. Can we then rank the welfare of each community in every generation across the two economies? Note that our analysis of the dynamic examples in Section 3.2.3 does indicate a negative answer to this question. The following proposition provides a more comprehensive response.

PRoposition 14: Assume that the RLM is of the linear homogeneous form. Denote the vector of feasible structural parameters and the initial conditions to be $\zeta$, with $\{\mu(\zeta), v(\zeta), \lambda(\zeta)\}$ being the coefficients of the $R L M$ in $\zeta$. Given $\zeta$, let $W_{i}^{*}(t \mid \zeta)$ be community $i$ 's equilibrium payoff (butter consumption) in generation $t$. Consider two feasible vectors $\left\{\zeta^{\prime}, \zeta^{\prime \prime}\right\}$ such that $\zeta^{\prime}$ induces an equilibrium path that devolves to perpetual anarchy, while $\zeta^{\prime \prime}$ induces an equilibrium path that attains perfect property rights, and $\left[\mu\left(\zeta^{\prime}\right)+v\left(\zeta^{\prime}\right)\right] \leq\left[\mu\left(\zeta^{\prime \prime}\right)+v\left(\zeta^{\prime \prime}\right)\right]$. Then there exists a finite $T$ such that for all $t>T,\left[W_{i}^{*}\left(t \mid \zeta^{\prime \prime}\right)\right]>\left[W_{i}^{*}\left(t \mid \zeta^{\prime}\right)\right]$ for $i=1,2$. On the other hand, there can exist one (or more) generation(s) $\tau \in[1, T]$ such that $\left[W_{i}^{*}\left(\tau \mid \zeta^{\prime}\right)\right]>\left[W_{i}^{*}\left(\tau \mid \zeta^{\prime \prime}\right)\right]$ for $i=1,2 .{ }^{57,58}$

Proposition 14 clarifies the 'utility trade-off' between the alternative $\pi$-trajectories in the short-run and in the long-run. Under a time-path that attains perfect property rights, there will be no inefficient guns investment once perfect property rights have been attained. In contrast, a time-path that devolves to anarchy will perpetually involve inefficient guns investment by both communities, and this intra-generational inefficient use of resources will also adversely affect long-run utility growth. But there can be a countervailing effect in the short-run. When the poorer community possesses a relatively small resource base, then aggregate investment in guns is also small. So, the rapid 'shrinking' of the poorer community along a time-path in which $\pi$ falls continually might be better for efficiency in the short-run (as compared to the case where the poorer community becomes relatively larger over time potentially leading to larger guns investments in the short-run). ${ }^{59}$

\footnotetext{
${ }^{57}$ These results hold so long as $\left[\mu\left(\zeta^{\prime}\right)+v\left(\zeta^{\prime}\right)\right]$ is 'not much greater' than $\left[\mu\left(\zeta^{\prime \prime}\right)+v\left(\zeta^{\prime \prime}\right)\right]$ (see the proof).

58 One might think that a more valid comparison between two economies E1 and E2 (as described above) should involve them having the same initial conditions at the common initial date $t=1$, and differ in some (or all) of the structural parameters. Note that Proposition 14 does not preclude such a comparison (the results will continue to be valid). In fact, the proposition establishes a stronger result that even if economy E1 started with much worse initial conditions, community welfare would unambiguously be higher in E1 in the long-run as long as the 'growth parameter' $(\mu+v)$ was not much bigger in E2 as compared to E1. ${ }^{59}$ The following import of Proposition 14 should also be noted. Suppose that we observe two multicommunity economies E1 and E2 evolving from a date at which they had 'similar attributes' (especially in terms of aggregate resources and initial rights regime), and we see that the endogenously chosen property
} 
The above analysis suggests that under a linear-homogeneous $R L M$, "permanently lifting the system' at any point in time to the perfect property rights regime will be better for the residents of all communities in the long-run. We now explore this issue in greater detail, focusing on both the long-run and the short-run. Specifically, returning to the scenario of a single economic system made up of two communities, we ask the question: Suppose that at the beginning of a particular generation $t^{[1]}$, a social planner could compel the two communities to perpetually set $\pi=1$, but could neither affect any other parameter of the model nor the guns-and-butter choices of the two communities. What would be the impact of the social planner's decision on the welfare of the two communities in every future generation? ${ }^{60}$ Our next result answers as follows.

Proposition 15: Assume that the RLM is of the linear homogeneous form, and there is no institutional inertia. Consider a vector $\zeta$ of feasible structural parameters and initial conditions that induces an equilibrium path which devolves to perpetual anarchy. Suppose that the social planner's intervention occurs in generation $t^{[1]} \geq 1$ and sets $\pi(t)=1$ for all $t \geq t^{[1]}$. For any $t \geq t^{[1]}$, let $W_{i}^{*}(t \mid \zeta)$ be community $i$ 's equilibrium payoff if the social planner had never intervened, and let $W_{i}^{[1]}(t \mid \zeta)$ be community $i$ 's equilibrium payoff given that the social planner has intervened in generation $t^{[1]}$. Then $W_{1}{ }^{[1]}(t \mid \zeta)>W_{1} *(t \mid \zeta)$ and $\left\{\left[W_{1}{ }^{[1]}(t \mid \zeta)\right]+\left[W_{2}{ }^{[1]}(t \mid \zeta)\right]\right\}>\left\{\left[W_{1}^{*}(t \mid \zeta)\right]+\left[W_{2}^{*}(t \mid \zeta)\right]\right\}$ for all $t \geq t[1]$; and there exists a finite $T \geq t^{[1]}$ such that for all $\tau>T:\left[W_{2}^{[1]}(\tau \mid \zeta)\right]>\left[W_{2}{ }^{*}(\tau \mid \zeta)\right]$. In contrast, $\left[W_{2}{ }^{*}\left(t^{[1]} \mid \zeta\right)\right]>\left[W_{2}{ }^{[1]}\left(t^{[1]} \mid \zeta\right)\right]$, and there can exist one (or more) additional generation(s) $t \in\left(t^{[1]}, T\right]$ such that $\left[W_{2}^{*}(t \mid \zeta)\right]>\left[W_{2}^{[1]}(t \mid \zeta)\right]$.

Proposition 15 clarifies the benefits and costs of a social intervention of permanently lifting a multicommunity system to the perfect property rights regime in a scenario where the structure of the linear-homogenous $R L M$ and other feasible conditions are such that the communities would devolve to anarchy under laissez faire. ${ }^{61}$ The richer community 1 and the two-community system

rights is continually strengthening in E1 and continually weakening in E2. Then if we currently find individual community welfare to be higher in the E1 communities than in the E2 communities, we should be confident that this welfare ranking will persist in the future. But if we currently see the opposite welfare ranking between the economies, we should recognize that the ranking might get reversed in the future.

${ }^{60}$ We have consistently maintained that our two-community system lacks an external enforcer of laws. So the sudden introduction of a 'social planner' might be a bit jarring to the reader. (S)he is, however, free to 'replace' the social planner by an 'elders' council' with its members being elders from both communities.

${ }^{61}$ The conclusions of Proposition 15 might not hold for other $R L M$ structures. For instance, consider the autonomous $R L M$ with $m \geq 1$ (see Proposition 9), and suppose that $\rho(1) \leq \rho^{-}(\sigma)$ and that there is no institutional inertia. In that case, if the social planner intervenes in period $t^{[1]} \geq 1$, the resources of the two communities in any generation $t>t^{[1]}$ will be independent of past history and past intervention. Then, with $\pi^{*}(t)=0$ and $\pi^{[1]}(t)=1$ for all $t \geq t^{[1]}$, in every generation $t \geq t^{[1]}$ the poorer community will be strictly worse off as compared to laissez faire: $W_{2}{ }^{*}(t)=W_{2}^{\dagger}\left(\sigma, 0, R^{*}(t), \rho^{*}(t)\right)>\left[\rho^{*}(t) \cdot R^{*}(t)\right]=\left[\rho^{[1]}(t) \cdot R^{[1]}(t)\right]=W_{2}^{[1]}(t)$. 
as a whole will both be better-off in every generation as a result of this social intervention. But the welfare consequences will be 'mixed' for the poorer community 2 in the following sense: in the long-run the community 2 residents will indeed be better off, but in the generation of intervention $t^{[1]}$ - the community 2 residents will be worse off; further, in some future generations 'close enough to $t^{[1]}$, the poorer community 2 residents might be worse off due to the intervention.

To see more clearly the welfare effect of the intervention on the community 2 residents across generations, compare the following dynamic examples [a] and [b]. Recall that in Section 3.2.3, we compared Examples A and B in each of which we studied how the equilibrium welfare levels of the two communities evolved over time under two distinct $R L M$ structures: structure [I] with $\lambda=0.4$ (that led to attainment of perfect property rights), and structure [II] with $\lambda=0.8$ (that caused devolution to anarchy). We saw that with $\lambda=0.8$ there was greater inefficiency in the long-run, but not necessarily in the short-run. Here, we restrict attention to $R L M$ structure [II], and consider an intervention by a social planner where the perfect property rights regime is imposed upon the interactions between the two communities in all generations. We want to compare welfare outcomes (both in the long-run and in the short-run) of such an intervention to the welfare outcomes under laissez faire. In both examples [a] and [b], we set $\lambda=0.8$ and $\sigma=1 / 2$, and assume that there exists no institutional inertia (i.e., $\Pi(1)=[0,1]$ with $\varepsilon=\eta=1$ ). But our examples differ in the extent of initial resource inequality: for example [a] we take $R_{1}(1)=650$ and $R_{2}(1)=350$, while for example [b] we take $R_{1}(1)=830$ and $R_{2}(1)=170$.

Table 3[a] : Example [a]

\begin{tabular}{|c|c|c|c|c|c|c|c|c|c|c|}
\hline & & $t=1$ & $t=25$ & $t=50$ & $t=75$ & $\ldots$ & $t=250$ & $t=500$ & $t=750$ & $t=1000$ \\
\hline \multirow[t]{2}{*}{$W_{1}^{*}(t)$} & (i) $\pi^{*}(t)$ & 550 & 1026 & 1940 & 3618 & & $1.3 \times 10^{5}$ & $3.2 \times 10^{7}$ & $8.9 \times 10^{9}$ & $2.5 \times 10^{12}$ \\
\hline & (ii) $\pi=1$ & 650 & 1321 & 2767 & 5793 & & $1.0 \times 10^{6}$ & $1.7 \times 10^{9}$ & $2.7 \times 10^{12}$ & $4.3 \times 10^{15}$ \\
\hline \multirow[t]{2}{*}{$W_{2}^{*}(t)$} & (i) $\pi^{*}(t)$ & 355 & 668 & 1282 & 2432 & & $1.3 \times 10^{5}$ & $2.4 \times 10^{7}$ & $6.7 \times 10^{9}$ & $1.9 \times 10^{12}$ \\
\hline & (ii) $\pi=1$ & 350 & 711 & 1490 & 3119 & & $5.5 \times 10^{5}$ & $8.9 \times 10^{8}$ & $1.4 \times 10^{12}$ & $2.3 \times 10^{15}$ \\
\hline
\end{tabular}

Table 3[b] : Example [b]

\begin{tabular}{|c|c|c|c|c|c|c|c|c|c|c|}
\hline & & $t=1$ & $t=25$ & $t=50$ & $t=75$ & $\ldots$ & $t=250$ & $t=500$ & $t=750$ & $t=1000$ \\
\hline \multirow[t]{2}{*}{$W_{1} *(t)$} & (i) $\pi^{*}(t)$ & 333 & 582 & 1043 & 1861 & & $9.9 \times 10^{4}$ & $2.8 \times 10^{7}$ & $7.9 \times 10^{9}$ & $2.2 \times 10^{12}$ \\
\hline & (ii) $\pi=1$ & 830 & 1687 & 3533 & 7397 & & $1.3 \times 10^{6}$ & $2.1 \times 10^{9}$ & $3.4 \times 10^{12}$ & $5.5 \times 10^{15}$ \\
\hline \multirow[t]{2}{*}{$W_{2}^{*}(t)$} & (i) $\pi^{*}(t)$ & 333 & 540 & 906 & 1541 & & $7.5 \times 10^{4}$ & $2.1 \times 10^{7}$ & $5.9 \times 10^{9}$ & $1.7 \times 10^{12}$ \\
\hline & (ii) $\pi=1$ & 170 & 346 & 724 & 1515 & & $2.7 \times 10^{5}$ & $4.3 \times 10^{8}$ & $7.0 \times 10^{11}$ & $1.1 \times 10^{15}$ \\
\hline
\end{tabular}

Tables $3[\mathrm{a}]$ and $3[\mathrm{~b}]$ depict the equilibrium welfare levels of the two communities $\left\{W_{1} *(t), W_{2}^{*}(t)\right\}$ for different generations $t$ in two cases: (i) when the rights regime $\pi^{*}(\mathrm{t})$ is established under laissez 
faire, and (ii) when the perfect rights regime $\pi=1$ is imposed by the social planner in every generation. The tables clarify the following possibilities: For different levels of initial resource inequality (covering both Examples [a] and [b]), in the long-run (for all $t>100$ in our examples) it is the case that that the social planner's intervention is associated with higher community welfare for each of the communities (as compared to under laissez faire). In fact, the richer community is better off under the social planner's intervention in all generations in both examples. In contrast, in the short-run, equilibrium welfare outcomes for the poorer community can be 'contradictory' depending upon initial conditions. Specifically, in example [b], where there is significant initial resource inequality, it is the laissez faire scenario that is associated with higher community welfare for the poorer community for at least seventy-five generations following the intervention. ${ }^{62}$

In the context of example $[\mathrm{b}]$, Table $3[\mathrm{~b}]$ also throws light on the following issue: Suppose that the social planner (or the set of community elders playing the role of a social planner) could impose in period 1 'perpetual perfect rights ( $\pi=1$ for all $t \geq 1)$ ', and cared only about the welfare of the residents of the poorer community 2 . How would the social planner's myopia (or lack thereof) affect her decision-making? Certainly she would not want to impose the policy in period 1 if she herself was completely myopic (and cared only about the current generation). On the other hand, she would definitely want to initiate the policy in period 1 if she cared sufficiently about the welfare of very distant progenies. The striking fact that example $[\mathrm{b}]$ reveals is that even if the social planner was not completely myopic and cared sufficiently about the welfare of a set of progenies of the first generation residents, she would still not want to impose perpetual perfect rights in period 1 . This fact suggests the following possibility: if the community residents were themselves choosing whether to permit an external agency to enforce perfect property rights in every generation, and if each generation of residents cared about their immediate progeny to a limited extent, the first generation residents of community 2 would not make that interventionist choice. ${ }^{63}$

\footnotetext{
${ }^{62}$ The intuition behind these findings is as follows. With low initial resource inequality, there is quickly increasing conflict intensity over time, and so the downside of laissez faire is high. Further, the upside of the intervention by the social planner is also high because then resource inequality is held constant at a relatively small level forever (thus providing the poorer community with greater benefits from the aggregate resource pool). With high initial resource inequality, the initial downside of laissez faire for the poorer community is low since its optimal rights choice is $\pi=0$, while intervention immediately sets $\pi=1$. This greater initial divergence between optimal rights and imposed rights leads to slower convergence between the evolving welfare outcomes of the poorer community (under laissez faire and under social intervention).

${ }^{63}$ It is not possible to modify our analysis to study a dynastic model where each generation $t$ in each community $i$ cares about $\left[W_{i}(t)+\delta . W_{i}(t+1)\right]$ for some $\delta>0$, since in that case our approach of piecing together the $S P N E$ of each stand-alone generational game has to be abandoned. But note that if the following was true in the 'correctly worked out' dynastic model: The SPNE was unique for all $\delta$ 'close enough' to zero and exhibited continuity at $\delta=0$. Then for the parameter configuration in example [b], the initial generations in community 2 would reject the policy intervention as long as $\delta$ was close to zero.
} 


\section{Relation to the Literature}

In a sequence-of-generations state-of-nature model of appropriative conflicts, our central results are about the equilibrium co-evolution of endogenously chosen property rights enforcement regimes and community resources and welfare. We focus on the critical dependence of the equilibrium property rights path on initial and continuing resource inequality between contesting communities. In this dynamic setting, we show that even though imperfect property rights lead to welfare losses in every generation, the economic actors may not collectively succeed in continually strengthening the rights regime in order to attain perfect property rights. The problem lies with the (relatively) resource-poor community, which always has a weaker incentive to strengthen property rights, and can sometimes have a strict preference for weakening such rights. In fact, when initial resourceinequality is significant and/or the level of past productive investments is the dominant driver of resource-growth, the poorer community can drag the richer community into perpetual anarchy. These principal conclusions of our analysis complement existing results on appropriative conflicts and on the collective choice of property-rights regimes in the extant literature in the following ways. Our results for the guns-and-butter game - especially regarding the presence of the Hirshleiferian paradox of power - corroborate existing results on appropriative conflicts; see the survey by Garfinkel and Skaperdas (2007). However, in contrast to existing studies, we have focused not only on the influence of the magnitude of the security parameter $\pi \in[0,1]$ on the guns-and-butter equilibrium, but also on the consequent $\pi$-preference that the contesting communities come to hold. It is in this context that we have established possible anarchy-preference of the relatively-poorer community when resource inequality is substantial.

Then, in the sequence-of-generations model, it is by tracking the generational resource inequality and its evolution over time (impacted by the resource law-of-motion), that we have been able to delineate alternative evolution paths of the endogenously chosen property-rights regime; to the best of our knowledge, this exercise has not been carried out before. Our research most directly complements and extends the works of Genicot and Skaperdas (2002), Kumar (2008), and Skaperdas, McBride, and Milante (2011). These papers also study the endogenous choice of the security parameter $\pi$ in dynamic state-of-nature models of appropriative conflicts. ${ }^{64}$ But they posit (either implicitly or explicitly) the contestants to have identical initial resources and consider the process of building state capacity (i.e., strengthening $\pi$ ) to be costly. As a result, one of the central conclusions emanating from these papers is the following: The greater is the contestants' total wealth, the more likely is it that property rights enforcement will improve. ${ }^{65}$

\footnotetext{
${ }^{64}$ Genicot and Skaperdas (2002) study a two-period model while Skaperdas et. al. (2011) study a discretetime infinite-horizon model. In contrast, Kumar (2008) analyzes a continuous-time differential game.

${ }^{65}$ Gradstein (2004) reaches a similar conclusion in a neo-classical growth model where property-rights
} 
In our paper, in contrast, strengthening of property rights only needs mutual consent (and no resources); what matters here is not the magnitude of the total resources in the two-community economy, but how the resources are distributed between the communities in every generation. Further, the causation between evolving resource inequality and property-rights choices is not unidirectional; a current generation's rights choice does affect its progeny's resource distribution. In establishing how the evolution of resource inequality shapes the property-rights time path and vice versa, we also add to the Demsetzian insight that (enforcement of) property rights improve(s) when the benefits of doing so outweigh the costs. Demsetz (1967) provided historical examples where communities worked towards improving their property rights enforcement as the economic return from increased enforcement grew over time; Boyce and Bruner (2012) present a theoretical model of the Demsetzian argument. We complement the Demsetzian logic by arguing that the evolution of property rights enforcement can depend not only on the level of return from stronger property rights but also on its distribution: dynamically changing distribution of economic returns from altering rights enforcement can affect the evolution of endogenously chosen property-rights enforcement in important and unexpected ways.

In this context, it is worthwhile to note that in their paper on the origins of state capacity, Besley and Persson (2009) conclude that 'common interest public goods' are conducive to building state capacity. We complement this conclusion by demonstrating that when a group of economic agents hold significantly divergent preferences over a public good, collective action might not only fail to build state capacity but actually work towards destroying such capacity. Our model has identified unequal distribution and evolution of resource endowments to be a critical factor in generating such divergent preferences. It will be worthwhile, in future research, to identify other important factors that may cause serious divergence of preferences among economic citizens over collective goods.

\section{Appendix}

\section{PROOF OF LEMMA 1:}

We first consider the case in which $B_{k}=0$ for some $k \in\{1,2\}$. In this case, there is a unique equilibrium: $k$ will have nothing to defend and will set all its guns to attack, and its rival will have nothing to attack and will set all its guns to defense. Then the unique continuation payoff to each community $i(i=1,2)$ in this $S P N E$ will be: $\left\{\pi B_{i}+(1-\pi)\left[G_{i} /\left(G_{1}+G_{2}\right)\right] \sigma\left[B_{1}+B_{2}\right]\right\}$ with $B_{k}=0$. Henceforth, we take each of $B_{1}, G_{1}, B_{2}, G_{2}$ to be strictly positive. Also, let $i, j \in\{1,2\}$ and $i \neq j$. We first establish that in any equilibrium, it must be the case that $A_{i}^{\dagger}+D_{i}^{\dagger}=G_{i}$ for $i=1,2$. Note 
that we cannot have $A_{j}^{\dagger}=D_{j}^{\dagger}=0$ for any $j \in\{1,2\}$ since community $j$ can strictly gain by deviating to $A_{j}^{\dagger}>0$. Hence, $0<\left[A_{j}^{\dagger}+D_{j}^{\dagger}\right] \leq G_{j}$ for each $j$. Further, we cannot have $\left[A_{j}^{\dagger}+D_{j}^{\dagger}\right]<G_{j}$ for any $j$ since if $j$ 's opponent has strictly positive defensive weapons, then $j$ can do better by transforming any unused guns into attacking weapons, while if $j$ 's opponent has strictly positive attacking weapons, then $j$ can do better by transforming any unused guns into defensive weapons. Hence, in what follows, we take $D_{i}=G_{i}-A_{i}$ for $i=1,2$ and consider $A_{i}$ as community $i$ 's lone choice variable. We first look for the existence of 'interior equilibria' in which $A_{1}^{\dagger} \in\left(0, G_{1}\right)$ and $A_{2}^{\dagger} \in\left(0, G_{2}\right)$. Note that in such an equilibrium, each community $i$ must choose $A_{i}$ to maximize $v_{i}\left(A_{i}, A_{j}\right) \equiv \pi \cdot B_{i}+(1-$ $\pi)\left[D_{i} /\left(D_{i}+A_{j}\right)\right] \sigma . B_{i}+(1-\pi)\left[A_{i} /\left(D_{j}+A_{i}\right)\right] \sigma . B_{j}$. Straightforward differentiation shows that $v_{i}\left(A_{i}, A_{j}\right)$ is strictly concave in $A_{i}$ for all $A_{j} \in\left(0, G_{j}\right)$. Thus, if $\left\{A_{1}^{\dagger}, A_{2}^{\dagger}\right\} \in\left(0, G_{1}\right) \times\left(0, G_{2}\right)$ is an interior Nash equilibrium attack vector in the terminal conflict phase, then $\left\{A_{1}^{\dagger}, A_{2}^{\dagger}\right\}$ simultaneously solves: $\partial v_{1}\left(A_{1}^{\dagger}, A_{2}^{\dagger}\right) / \partial A_{1}=0$ and $\partial v_{2}\left(A_{2}^{\dagger}, A_{1}^{\dagger}\right) / \partial A_{2}=0$.

There exists a unique solution to the system of equations: $\partial v_{1}\left(A_{1}^{\dagger}, A_{2}^{\dagger}\right) / \partial A_{1}=0$ and $\partial v_{2}\left(A_{2}^{\dagger}, A_{1}^{\dagger}\right) / \partial A_{2}$ $=0$. From the first-order conditions of maximization, we have $A_{2}^{\dagger} B_{1}=D_{2}^{\dagger} B_{2}\left(D_{1}^{\dagger}+A_{2}^{\dagger}\right)^{2} /\left(D_{2}^{\dagger}+\right.$ $\left.A_{1}^{\dagger}\right)^{2}$, and $A_{1}^{\dagger} B_{2}=D_{1}^{\dagger} B_{1}\left(D_{2}^{\dagger}+A_{1}^{\dagger}\right)^{2} /\left(D_{1}^{\dagger}+A_{2}^{\dagger}\right)^{2}$. These two relations imply that: $A_{1}^{\dagger} / D_{1}^{\dagger}=B_{2} / B_{1}=$ $D_{2}^{\dagger} / A_{2}^{\dagger}$. So, given $D_{i}^{\dagger}+A_{i}^{\dagger}=G_{i}$ for $i=1$, 2, we conclude that there exists a continuation Nash equilibrium in the terminal conflict phase (which also is the unique interior Nash equilibrium) in which the attack-defense split of each community $i$ is: $\left\{A_{i}^{\dagger}=\left[B_{j} /\left(B_{i}+B_{j}\right)\right] G_{i} ; D_{i}^{\dagger}=\left[B_{i} /\left(B_{i}+B_{j}\right)\right] G_{i}\right\}$. Next, we argue that a 'corner equilibrium' in which at least one community $i$ sets $A_{i}^{\dagger}=0$ or $A_{i}^{\dagger}=G_{i}$ cannot exist. Note that there cannot exist an equilibrium with $A_{i}^{\dagger}=0$ for any $i$, because while $A_{j}=$ $G_{j}$ is the unique best response to $A_{i}=0, A_{i}=0$ is not a best response to $A_{j}=G_{j}$. Further, no equilibrium with $A_{i}^{\dagger}=G_{i}$ and $A_{j}^{\dagger}=a>0$ can exist because $A_{j}=0.5 a$ is a better response to $A_{i}=G_{i}$.

Thus, we conclude that when $B_{1}, G_{1}, B_{2}, G_{2}$ are all strictly positive, there exists a unique equilibrium in which the attack-defense split of each community $i$ is $\left\{A_{i}^{\dagger}=\left[B_{j} /\left(B_{i}+B_{j}\right)\right] G_{i} ; D_{i}^{\dagger}=\left[B_{i} /\left(B_{i}+B_{j}\right)\right] G_{i}\right\}$. Then it is easy to verify that the unique continuation equilibrium payoff to each community $i$ will be $\left\{\pi B_{i}+(1-\pi)\left[G_{i} /\left(G_{i}+G_{j}\right)\right] \sigma\left[B_{i}+B_{j}\right]\right\}$.

\section{ProOF OF PROPOSITION 2:}

The proof of part [B] follows immediately, so we just consider part [A] here. Let $i, j \in\{1,2\}, i \neq j$, and $\pi<1$. At the outset, we note that the following claims are true: (i) In any $S P N E$, it must be that $B_{1}^{\dagger}+G_{1}^{\dagger}=R_{1}$ and $B_{2}^{\dagger}+G_{2}^{\dagger}=R_{2}$ since for any community $i$, setting $B_{i}^{\dagger}+G_{i}^{\dagger}<R_{i}$ is a strictly dominated strategy. (ii) In any SPNE, it cannot be that $B_{1}^{\dagger}=B_{2}^{\dagger}=0$ because then the continuation payoff to each community will be zero (while any community $i$ can guarantee a positive payoff for itself by setting, say, $B_{i}^{\dagger}=G_{i}^{\dagger}=0.5 R_{i}$ ). (iii) Given $\pi<1$, in any $S P N E$, it cannot be that $G_{1}^{\dagger}=G_{2}^{\dagger}$ $=0$ because then any community $i$ can deviate and invest an infinitesimal amount in guns and steal a discrete amount of rival butter. (iv) Given $\pi<1$, in any $S P N E$, it cannot be that $G_{i}^{\dagger}=0$ while $G_{j}^{\dagger}=$ $g>0$ because $G_{j}=0.5 g$ is a better response to $G_{i}=0$. 
In the remainder of the proof, we restrict attention to the case: $B_{1}+B_{2}>0$ and $G_{1} \times G_{2}>0$. Then the statement of Lemma 1 holds, and the continuation equilibrium butter bribe $b_{12}$ is determined directly from the fact that the Nash Bargaining Solution will ensure that each community gets a negotiation payoff of \{half of net bargaining surplus + own outside option\}.

Thus each community $i$, given its conjecture about $G_{j} \in\left(0, R_{j}\right)\left(\right.$ resp. $\left.G_{j}=R_{j}\right)$, must maximize over all $G_{i} \in\left(0, R_{i}\right]$ (resp., $\left.\left[0, R_{i}\right]\right), V_{i}\left(G_{i}, G_{j}\right) \equiv\left\{0.5(1-\pi)(1-\sigma)\left(B_{i}+B_{j}\right)\right\}+\left\{\pi \cdot B_{i}+(1-\pi)\left[G_{i} /\left(G_{i}+G_{j}\right)\right] \sigma\left[B_{i}+B_{j}\right]\right\}$ where $G_{k}+B_{k}=R_{k}$ for $k=1,2 .{ }^{66}$ Note that $V_{i}\left(G_{i}, G_{j}\right)$ is strictly concave in $G_{i} \in\left(0, R_{i}\right]$ (resp. [0, $\left.R_{i}\right]$ ) for all $G_{j} \in\left(0, R_{j}\right)$ (resp. $\left.G_{j}=R_{j}\right)$. As a result, all $\left\{G_{1}^{\dagger}, G_{2}^{\dagger}\right\} \in\left(0, R_{1}\right) \times\left(0, R_{2}\right)$ that simultaneously solve: $\partial V_{1}\left(G_{1}^{\dagger}, G_{2}^{\dagger}\right) / \partial G_{1}=0$ and $\partial V_{2}\left(G_{2}^{\dagger}, G_{1}^{\dagger}\right) / \partial G_{2}=0$ will be 'interior' equilibria and the only such equilibria. We determine that $\left\{G_{1}^{\dagger}=G_{2}^{\dagger}=\tilde{\rho}() R.\right\}$ is the unique solution to $\partial V_{1}(.) / \partial G_{1}=\partial V_{2}(.) / \partial G_{2}=$ $0 .^{67}$ Thus, when $R_{2}>\tilde{\rho}(\pi \mid \sigma) R,\left\{G_{1}^{\dagger}()=.G_{2}^{\dagger}()=.\tilde{\rho}(\pi \mid \sigma) R\right\}$ will be the unique interior equilibrium guns investment, and when $R_{2} \leq \tilde{\rho}(\pi \mid \sigma) R$, there will not exist any interior equilibrium.

Next, we prove that when $R_{2}>\tilde{\rho}()$.$R , there is no other 'corner equilibrium'. The best response$ function $G_{i}{ }^{*}\left(G_{j}\right)$ reaches its maximum when $G_{j}=\tilde{\rho}(.) \cdot R$, and that $G_{i}{ }^{*}\left(G_{j}=\tilde{\rho}(.) \cdot R\right)=\tilde{\rho}(.) \cdot R$. The maximal value $=\tilde{\rho}(.) \cdot R$, is necessarily smaller than $R_{1}$, and is greater than $R_{2}$ if and only if $\rho \leq \tilde{\rho}($.$) .$ Thus when $R_{2}>\tilde{\rho}(\pi \mid \sigma) R$, there will be no equilibrium other than $\left\{G_{1}^{\dagger}()=.G_{2}^{\dagger}()=.\tilde{\rho}(\pi \mid \sigma) R\right\}$.

Finally, we identify equilibria when $R_{2} \leq \tilde{\rho}()$.$R . We first show that \left\{G_{1}^{\dagger}()=.\left\{\sqrt{ }\left[4 \tilde{\rho}(.) \cdot R \cdot R_{2}\right]-R_{2}\right\}\right.$, $\left.G_{2}^{\dagger}()=.R_{2}\right\}$ is an equilibrium. By determining the value of $G_{1}$ for which $\partial V_{1}\left(G_{1}, G_{2}\right) / \partial G_{1}=0$ when evaluated at $G_{2}=R_{2}$, we obtain: $G_{1} *\left(G_{2}=R_{2}\right)=\left\{\sqrt{ }\left[4 \tilde{\rho}(.) \cdot R \cdot R_{2}\right]-R_{2}\right\}$. It is easily verified that $0<$ $G_{1}{ }^{*}\left(G_{2}=R_{2}\right)<R_{1}$. This is the unique best response to $G_{2}=R_{2}$ as $V_{1}\left(G_{1}, G_{2}=R_{2}\right)$ is strictly concave in $G_{1} \in\left[0, R_{1}\right]$. Further, for $G_{2}>0$ we have: $\partial V_{2}\left(G_{2}, G_{1}\right) / \partial G_{2}=(1-\pi) \cdot\left[G_{1} /\left(G_{1}+G_{2}\right)^{2}\right] \sigma .\left[B_{1}+B_{2}\right]-$ $(1-\pi) \cdot\left[G_{2} /\left(G_{1}+G_{2}\right)\right] \sigma-(1-\pi) .(1-\sigma) / 2-\pi$. It can be shown after some algebraic work that $\partial V_{2}\left(G_{2}, G_{1}\right) / \partial G_{2}$ evaluated at $G_{2}=R_{2}$ and $G_{1}=\left\{\sqrt{ }\left[4 \tilde{\rho}(.) \cdot R \cdot R_{2}\right]-R_{2}\right\}$ is strictly positive for all $R_{2}<$ $\tilde{\rho}()$.$R , and is zero when R_{2}=\tilde{\rho}()$.$R . Further, V_{2}\left(G_{2}, G_{1}\right)$ is strictly concave in $G_{2} \in\left(0, R_{2}\right] .{ }^{68}$ Hence, we conclude that when $R_{2} \leq \tilde{\rho}(\pi \mid \sigma) R$, then $\left\{G_{1}^{\dagger}()=.\left\{\sqrt{ }\left[4 \tilde{\rho}(\pi \mid \sigma) \cdot R \cdot R_{2}\right]-R_{2}\right\}, G_{2}^{\dagger}()=.R_{2}\right\}$ will be an equilibrium.

${ }^{66}$ Note that if $G_{j} \in\left(0, R_{j}\right)$ and so $B_{j}>0$, then $V_{i}\left(G_{i}=0, G_{j}\right) \equiv\left\{0.5(1-\pi)(1-\sigma)\left(B_{i}\right)\right\}+\left\{\pi \cdot B_{i}+(1-\pi)\left[G_{i}\right.\right.$ $\left.\left./\left(G_{i}+G_{j}\right)\right] \sigma\left[B_{i}+B_{j}\right]\right\}$ where $G_{k}+B_{k}=R_{k}$ for $k=1,2$. Hence when $B_{j}>0, V_{i}\left(G_{i}, G_{j}\right)$ is discontinuous at $G_{i}=0$; however, $V_{i}\left(G_{i}=0, G_{j}\right)<V_{i}\left(G_{i}=\varepsilon>0, G_{j}\right)$, so we do not need to consider a deviation by community $i$ to $G_{i}=$ 0 while determining its best response to rival $G_{j} \in\left(0, R_{j}\right)$, and only need to maximize $V_{i}\left(G_{i}, G_{j}\right)$ over $G_{i} \in(0$, $\left.R_{i}\right]$. The above-described discontinuity in $V_{i}\left(G_{i}, G_{j}\right)$ at $G_{i}=0$ does not occur when rival $G_{j}=R_{j}$, and then we can maximize $V_{i}\left(G_{i}, G_{j}\right) \equiv\left\{0.5(1-\pi)(1-\sigma)\left(B_{i}+B_{j}\right)\right\}+\left\{\pi \cdot B_{i}+(1-\pi)\left[G_{i} /\left(G_{i}+G_{j}\right)\right] \sigma\left[B_{i}+B_{j}\right]\right\}$ over all $G_{i} \in\left[0, R_{i}\right]$.

${ }^{67}$ Specifically we have $\partial V_{i}\left(G_{i}, G_{j}\right) / \partial G_{i}=\left\{(1-\pi) \cdot\left[G_{j} /\left(G_{1}+G_{2}\right)^{2}\right] \sigma \cdot\left[B_{1}+B_{2}\right]-(1-\pi) \cdot\left[G_{i} /\left(G_{1}+G_{2}\right)\right] \sigma-\right.$ $(1-\pi) .(1-\sigma) / 2-\pi\}$ for $G_{j} \in\left(0, R_{j}\right]$. It follows that $V_{i}\left(G_{i}, G_{j}\right)$ is strictly concave in $G_{i}$ for all $G_{j} \in\left(0, R_{j}\right]$.

${ }^{68}$ Note that, given $0<G_{1}=G_{1} *\left(G_{2}=R_{2}\right)<R_{1}$, there is an inconsequential discontinuity in $V_{2}\left(G_{2}, G_{1}\right)$ at $G_{2}$ $=0$, and so we need not consider a deviation by community 2 to $G_{2}=0$. 
To conclude that there can be no other equilibrium when $R_{2} \leq \tilde{\rho}()$.$R , recall from above the following$ two facts: (i) no interior equilibrium can exist for $\rho \leq \tilde{\rho}\left(\right.$.), and (ii) no corner equilibrium with $G_{1}{ }^{\dagger}=$ $R_{1}$ can exist for $\rho \leq \tilde{\rho}\left(\right.$.) because community 1's best response can never be $G_{1} *\left(G_{2}\right)=R_{1}$ for any rival $G_{2}$. Given the existence of a unique equilibrium for all feasible parameters, the expression for the equilibrium butter bribe $b_{12}{ }^{\dagger}$ is given by: \{[Community 1's butter production (in equilibrium)][half of net bargaining surplus (in equilibrium) + Community 1's outside option (in equilibrium)]\}.

The unique $S P N E$ payoffs of the two communities (equivalently, their equilibrium aggregate butter consumptions), denoted by $W_{1}^{\dagger}(\sigma, \pi, R, \rho)$ and $W_{2}^{\dagger}(\sigma, \pi, R, \rho)$, are obtained by direct substitution of expressions for $G_{1}^{\dagger}(\sigma, \pi, R, \rho)$ and $G_{2}^{\dagger}(\sigma, \pi, R, \rho)$ in the payoff functions $V_{1}\left(G_{1}, G_{2}\right)$ and $V_{2}\left(G_{2}, G_{1}\right)$.

The proofs of Corollary 3, Propositions $4-6$, and Lemma 7 are relatively straightforward, even though some are quite tedious. These proofs are available from the authors.

\section{PROOF OF PROPOSITION 8:}

Suppose that the equilibrium path leads to high resource inequality. Then there exists $t^{\mathrm{H}} \geq 1$ such that $\rho^{*}(t) \leq \rho^{-}(\sigma)$ for all $t \geq t^{\mathrm{H}}$. We know that $\pi^{\dagger}(\rho \mid \sigma)=0$ for all $\rho \leq \rho^{-}(\sigma)$. So, for all $t \geq t^{\mathrm{H}}, \pi^{*}(t)$ $=s_{2}{ }^{*}(t)=\max \left\{\pi^{*}(t-1)-\varepsilon, 0\right\}$ where $\varepsilon$ is a strictly positive constant. So, there necessarily exists $t^{\mathrm{A}} \geq t^{\mathrm{H}}$ such that $\pi^{*}(t)=0$ for all $t \geq t^{\mathrm{A}}$. Hence, the equilibrium path devolves to perpetual anarchy. Alternatively, suppose the equilibrium path devolves to perpetual anarchy. Then there exists $t^{\mathrm{A}} \geq 1$ such that $\pi^{*}(t)=0$ for all $t \geq t^{\mathrm{A}}$. Note that if in some generation $t \geq t^{\mathrm{A}}$ we have $\rho^{*}(t)>\rho^{-}(\sigma)$, then we will also necessarily have: $\pi^{*}(t)>0$. But this is a contradiction, and so we will necessarily have $\rho^{*}(t) \leq \rho^{-}(\sigma)$ for all $t \geq t^{\mathrm{A}}$. In fact, there exists $t^{\mathrm{H}}$ with $1 \leq t^{\mathrm{H}} \leq t^{\mathrm{A}}$, such that $\rho^{*}(t) \leq \rho^{-}(\sigma)$ for all $t \geq t^{\mathrm{H}}$, implying that the equilibrium path leads to high resource inequality.

Next, suppose that the equilibrium path leads to low resource inequality. For all $\xi \in\left(0,\left[\rho^{+}(\sigma)-\right.\right.$ $\left.\left.\rho^{-}(\sigma)\right]\right)$, consider the function: $\left\{1-\pi^{\dagger}\left(\left[\rho^{+}(\sigma)-\xi\right] \mid \sigma\right)\right\} \in(0,1)$. Note that it is a strictly increasing function of $\xi$, which approaches 0 as $\xi$ approaches 0 , and which approaches 1 as $\xi$ approaches $\left[\rho^{+}(\sigma)-\rho^{-}(\sigma)\right]$. In particular, this implies that $\left\{1-\pi^{\dagger}\left(\left[\rho^{+}(\sigma)-\xi\right] \mid \sigma\right)\right\}$ is an invertible function. Then, for all $\psi \in(0,1)$, we can define a function $\xi(\psi) \in\left(0,\left[\rho^{+}(\sigma)-\rho^{-}(\sigma)\right]\right)$ implicitly through: $\psi=\left\{1-\pi^{\dagger}\left(\left[\rho^{+}(\sigma)-\xi(\psi)\right] \mid \sigma\right)\right\}$. Pick some $\psi \in(0,1)$; our goal now is to show that there exists $t^{\mathrm{P}}(\psi) \geq 1$ such that $\pi^{*}(t) \geq[1-\psi]$ for all $t \geq t^{\mathrm{P}}(\psi)$. Consider the corresponding function $\xi(\psi)$. Given our assumptions, we know that there exists $t^{\mathrm{L}}(\xi(\psi)) \geq 1$, such that $\rho^{*}(t) \geq\left[\rho^{+}(\sigma)-\xi(\psi)\right]$ for all $t \geq$ $t^{\mathrm{L}}(\xi(\psi))$. Suppose that in some period $t \geq t^{\mathrm{L}}(\xi(\psi))$, we have: $(i)\left[\pi^{*}(t-1)+\eta\right]<\pi^{\dagger}\left(\left[\rho^{+}(\sigma)-\xi(\psi)\right] \mid \sigma\right)$, then we will necessarily have: $\pi^{*}(t)=\left[\pi^{*}(t-1)+\eta\right]$, while if we have: $(i i)\left[\pi^{*}(t-1)+\eta\right] \geq$ $\pi^{\dagger}\left(\left[\rho^{+}(\sigma)-\xi(\psi)\right] \mid \sigma\right)$, then we will necessarily have: $\pi^{*}(t) \geq \pi^{\dagger}\left(\left[\rho^{+}(\sigma)-\xi(\psi)\right] \mid \sigma\right)$. Putting $(i)$ and (ii) together it is easy to verify that there exists a period $t^{\mathrm{P}}(\psi)$ such that $\pi^{*}(t) \geq \pi^{\dagger}\left(\left[\rho^{+}(\sigma)-\xi(\psi)\right] \mid \sigma\right)$ $=\left[1-\left\{1-\pi^{\dagger}\left(\left[\rho^{+}(\sigma)-\xi(\psi)\right] \mid \sigma\right)\right\}\right]=[1-\psi]$, for all $t \geq t^{\mathrm{P}}(\psi)$. 
Alternatively, suppose that the equilibrium path attains perfect property rights. Then for all $\psi \in(0, \varepsilon)$ there exists $t^{\mathrm{P}}(\psi)$ such that $\pi^{*}(t) \geq[1-\psi]$ for all $t \geq t^{\mathrm{P}}(\psi)$. Then it can be verified that we cannot have $\rho^{*}(t)<\left[\rho^{+}(\sigma)-\xi(\psi)\right]$ for any $t \geq t^{\mathrm{P}}(\psi)$, where $\xi(\psi)$ is the same monotonic transformation as defined above. It then follows that for $\xi$ sufficiently close to 0 (to be precise, for all $\xi \in\left(0,\left[\rho^{+}(\sigma)-\rho^{\#}(\varepsilon)\right]\right)$, where $\rho^{\#}(\varepsilon)$ is implicitly defined by: $\left.\pi^{\dagger}\left(\rho^{\#}(\varepsilon) \mid \sigma\right)=[1-\varepsilon]\right)$, there exists $t^{\mathrm{L}}(\xi)$ such that $\rho^{*}(t) \geq\left[\rho^{+}(\sigma)-\xi\right]$ for all $\mathrm{t} \geq t^{\mathrm{L}}(\xi)$, implying eventual low resource inequality.

PROOFS OF PROPOSITIONS 9 AND 10:

We begin by establishing Proposition 10 .

[A] We first establish part (i). We will do so by showing that resource inequality will eventually persist at a sufficiently high level, such that $\rho^{*}(t) \leq 0.5 \sigma /[1+\sigma]$ for all $t$ beyond some finite generation. Then, since $0.5 \sigma /[1+\sigma]<\rho^{-}(\sigma)$, application of Proposition 8 will give the result.

It follows from Property F-1.1 that if $\rho(1) \leq 0.5 \sigma /[1+\sigma]$, then $\rho^{*}(t) \leq 0.5 \sigma /[1+\sigma]$ for all $t$. Thus, the result holds for $\rho(1) \leq 0.5 \sigma /[1+\sigma]$. Below, we consider the case when $\rho(1) \in\left(0.5 \sigma /[1+\sigma], \rho^{+}(\sigma)\right)$. Given $\rho(1) \in\left(0.5 \sigma /[1+\sigma], \rho^{+}(\sigma)\right)$, we first prove that along the equilibrium path, resource inequality strictly increases in the first few generations. Note that: $\rho(1) \in\left(0.5 \sigma /[1+\sigma], \rho^{+}(\sigma)\right) \Rightarrow \pi^{*}(1)<1$ $\Rightarrow \rho^{*}(2)<\rho(1)$, due to F-1.1. If $\rho^{*}(2)>0.5 \sigma /[1+\sigma]$, then the previous argument can be used again and we get: $\rho^{*}(3)<\rho^{*}(2)$. Thus, if $\rho^{*}\left(t^{\prime}\right)>0.5 \sigma /[1+\sigma]$ in some generation $t^{\prime} \geq 1$, then $\rho^{*}\left(t^{\prime}+1\right)<$ $\rho^{*}\left(t^{\prime}\right)$. Further, from F-1.1, if $\rho^{*}\left(t^{\prime \prime}\right) \leq 0.5 \sigma /[1+\sigma]$ in some $t^{\prime \prime} \geq 2$, then $\rho^{*}(t) \leq 0.5 \sigma /[1+\sigma]$ for all $t \geq$ $t^{\prime \prime}$. We argue, by contradiction, that the equilibrium path possesses this property.

So, suppose not, i.e., suppose $\rho^{*}(t)>0.5 \sigma /[1+\sigma]$, for all $t \geq 1$. Then, based on the property possessed by the equilibrium path that we established before - that if $\rho^{*}\left(t^{\prime}\right)>0.5 \sigma /[1+\sigma]$ then $\rho^{*}\left(t^{\prime}+1\right)<\rho^{*}\left(t^{\prime}\right)$ - it must be that: $\rho(1)>\rho^{*}(2)>\ldots>\rho^{*}(t)>\ldots>0.5 \sigma /[1+\sigma] \Rightarrow\left\{\rho^{*}(\mathrm{t})\right\}_{1}^{\infty}$ necessarily converges down to some $\rho^{\mathrm{C}} \in\left[0.5 \sigma /(1+\sigma), \rho(1)\right.$ ) (without ever exactly attaining $\rho^{\mathrm{C}}$ ), i.e., the equilibrium path is of the form: $\rho(1)>\rho^{*}(2)>\ldots>\rho^{*}(t)>\rho^{*}(t+1)>\ldots>\rho^{\mathrm{C}}$. Also, note that such a time-path will have: $\pi^{*}(t)<1$ and $R^{*}(t)>0$, for all $t$.

In order to show that the equilibrium path cannot actually converge (weakly) above $0.5 \sigma /[1+\sigma]$, we will define a specific function, note that this function is continuous in its argument and then show that the equilibrium path's properties (established above) necessarily imply that the function is discontinuous at some point in its domain. This provides us the needed contradiction.

Given $R>0$ and $\sigma \in[1 / 2,1)$, we define the function: $J(\rho \mid \sigma) \equiv r^{\dagger}\left(\sigma, \pi^{\dagger}(\rho \mid \sigma), R, \rho\right)$ for all $\rho \in$ $\left[0.5 \sigma /[1+\sigma], \rho^{+}(\sigma)\right)$. As $\boldsymbol{F}($.$) is homogeneous, J(\rho \mid \sigma)$ is independent of $R$. It is straightforward to establish that $J(\rho \mid \sigma)$ is continuous over its domain. ${ }^{69}$ Finally, Property F-1.1 implies that $J(\rho \mid \sigma)<$

${ }^{69}$ We establish here the continuity of $J(\rho \mid \sigma) \equiv r^{\dagger}\left(\sigma, \pi^{\dagger}(\rho \mid \sigma), R, \rho\right)$ over its domain $\rho \in[0.5 \sigma /[1+\sigma]$, $\left.\rho^{+}(\sigma)\right)$ as follows. Fix any $\sigma \in[0.5,1)$ and $R>0$, and let $i=1,2$. We have: $r^{\dagger}(\sigma, \pi, R, \rho) \equiv\{F(\rho R$, 
$\rho$ for all $\rho$. Since $\rho^{\mathrm{C}} \in\left[0.5 \sigma /[1+\sigma], \rho^{+}(\sigma)\right)$, we define $J\left(\rho^{\mathrm{C}} \mid \sigma\right) \equiv\left[\rho^{\mathrm{C}}-\chi\left(\rho^{\mathrm{C}} \mid \sigma\right)\right]$, where $\chi\left(\rho^{\mathrm{C}} \mid \sigma\right)>0$. We now state the following Claim: Given that the equilibrium path $\left\{\rho^{*}(\mathrm{t})\right\}_{t=1}^{\infty}$ converges to $\rho^{\mathrm{C}}$, and given $\{\eta>0, \varepsilon>0\}$, there must exist a finite generation $T$ such that $\pi^{*}(t)=\pi^{\dagger}\left(\rho^{*}(t) \mid \sigma\right)$ for all $t \geq T$. The proof of the Claim is as follows: there must necessarily exist some finite generation $T$ such that $\pi^{*}(t)=\pi^{\dagger}\left(\rho^{*}(t)\right)$ for all $t \geq T$. [In order to simplify notation here, we ignore the dependence of $\pi^{\dagger}($.) on $\sigma$.] Given the hypothesis of the Claim, note that there necessarily exists a finite generation $\tau$ such that for all $t \geq \tau$ : $\rho^{\mathrm{C}}<\rho^{*}(t)<\left[\rho^{\mathrm{C}}+\Delta\right]$, where $\left[\pi^{\dagger}\left(\rho^{\mathrm{C}}+\Delta\right)-\pi^{\dagger}\left(\rho^{\mathrm{C}}\right)\right]<\min \{\varepsilon, \eta\}$. Now, there are three cases to consider: $(i) \pi^{*}(\tau) \in\left[\pi^{\dagger}\left(\rho^{\mathrm{C}}\right), \pi^{\dagger}\left(\rho^{\mathrm{C}}+\Delta\right)\right]$, (ii) $\pi^{*}(\tau)<\pi^{\dagger}\left(\rho^{\mathrm{C}}\right)$, and (iii) $\pi^{*}(\tau)>$ $\pi^{\dagger}\left(\rho^{\mathrm{C}}+\Delta\right)$. We want to establish for all three cases that there must exist some generation $T \geq \tau$ such that $\pi^{*}(t)=\pi^{\dagger}\left(\rho^{*}(t)\right)$ for all $t \geq T$. First, we consider case $(i)$. Since $\rho^{\mathrm{C}}<\rho^{*}(t)<\left[\rho^{\mathrm{C}}+\Delta\right]$ for all $t \geq \tau$, hence: $\pi^{\dagger}\left(\rho^{C}\right) \leq \pi^{\dagger}\left(\rho^{*}(t)\right) \leq \pi^{\dagger}\left(\rho^{C}+\Delta\right)$ for all $t \geq \tau$. Now, since $\left[\pi^{\dagger}\left(\rho^{C}+\Delta\right)-\pi^{\dagger}\left(\rho^{C}\right)\right]<\min \{\varepsilon, \eta\}$, therefore for $t \geq \tau:\left|\left[\pi^{\dagger}\left(\rho^{*}(t)\right)-\pi^{\dagger}\left(\rho^{*}(t+1)\right)\right]\right|<\min \{\varepsilon, \eta\}$. So, if $\pi^{*}(t)=\pi^{\dagger}\left(\rho^{*}(t)\right)$ in some generation $t \geq \tau$, then $\pi^{*}(t+1)=\pi^{\dagger}\left(\rho^{*}(t+1)\right)$. Finally, since $\pi^{*}(\tau) \in\left[\pi^{\dagger}\left(\rho^{\mathrm{C}}\right), \pi^{\dagger}\left(\rho^{\mathrm{C}}+\Delta\right)\right]$ and $\pi^{\dagger}\left(\rho^{*}(\tau+1)\right) \in\left[\pi^{\dagger}\left(\rho^{\mathrm{C}}\right), \pi^{\dagger}\left(\rho^{\mathrm{C}}+\Delta\right)\right]$, therefore: $\left|\left[\pi^{*}(\tau)-\pi^{\dagger}\left(\rho^{*}(\tau+1)\right)\right]\right|<\min \{\varepsilon, \eta\}$. So, $\pi^{*}(\tau+1)=$ $\pi^{\dagger}\left(\rho^{*}(\tau+1)\right)$. Hence, $\pi^{*}(t)=\pi^{\dagger}\left(\rho^{*}(t)\right)$ for all $t \geq(\tau+1)$. Next, we consider case (ii). In any generation $t \geq \tau$, if $\pi^{*}(t-1)<\pi^{\dagger}\left(\rho^{*}(t)\right)$, then $\pi^{*}(t)=\min \left\{\left[\pi^{*}(t-1)+\eta\right], \pi^{\dagger}\left(\rho^{*}(t)\right)\right\}$. Since for $t \geq \tau$ : $\pi^{\dagger}\left(\rho^{\mathrm{C}}\right) \leq \pi^{\dagger}\left(\rho^{*}(t)\right) \leq \pi^{\dagger}\left(\rho^{\mathrm{C}}+\Delta\right)$, so there must exist a generation $\tau^{\prime} \geq \tau$ such that $\pi^{*}\left(\tau^{\prime}\right) \in\left[\pi^{\dagger}\left(\rho^{\mathrm{C}}\right)\right.$, $\left.\pi^{\dagger}\left(\rho^{\mathrm{C}}+\Delta\right)\right]$. Then, by reasoning given in case $(i)$, we have that $\pi^{*}(t)=\pi^{\dagger}\left(\rho^{*}(t)\right)$ for all $t \geq\left(\tau^{\prime}+1\right)$. Finally, we consider case (iii). In any generation $t \geq \tau$, if $\pi^{*}(t-1)>\pi^{\dagger}\left(\rho^{*}(t)\right)$, then $\pi^{*}(t)=$ $\max \left\{\left[\pi^{*}(t-1)-\varepsilon\right], \pi^{\dagger}\left(\rho^{*}(t)\right)\right\}$. Since for $t \geq \tau: \pi^{\dagger}\left(\rho^{C}\right) \leq \pi^{\dagger}\left(\rho^{*}(t)\right) \leq \pi^{\dagger}\left(\rho^{C}+\Delta\right)$, so there must exist a generation $\tau^{\prime \prime} \geq \tau$ such that $\pi^{*}\left(\tau^{\prime \prime}\right) \in\left[\pi^{\dagger}\left(\rho^{\mathrm{C}}\right), \pi^{\dagger}\left(\rho^{\mathrm{C}}+\Delta\right)\right]$. Then, by reasoning given in case $(i)$, we have that $\pi^{*}(t)=\pi^{\dagger}\left(\rho^{*}(t)\right)$ for all $t \geq\left(\tau^{\prime \prime}+1\right)$. This completes the proof of the Claim.

Given the Claim above, for all $t \geq T$, we have: $\rho^{*}(t+1)=r^{\dagger}\left(\sigma, \pi^{*}(t), R^{*}(t), \rho^{*}(t)\right)=r^{\dagger}\left(\sigma, \pi^{\dagger}\left(\rho^{*}(t) \mid\right.\right.$ $\left.\sigma), R^{*}(t), \rho^{*}(t)\right)=J\left(\rho^{*}(t) \mid \sigma\right)$, since $\rho^{*}(t) \in\left[0.5 \sigma /[1+\sigma], \rho^{+}(\sigma)\right)$. Summarizing, we have: $\rho^{*}(t+1)=$ $J\left(\rho^{*}(t) \mid \sigma\right)$ for all $t \geq T$. Recall that: $\rho(1)>\rho^{*}(2)>\ldots>\rho^{*}(t)>\rho^{*}(t+1)>\ldots>\rho^{\mathrm{C}}$. Hence: $J\left(\rho^{*}(t)\right.$ $\mid \sigma)>\rho^{\mathrm{C}}$, for all $t \geq T$. Since $\left\{\rho^{*}(\mathrm{t})\right\}_{t=1}^{\infty}$ converges down to $\rho^{\mathrm{C}}$, so $\rho^{*}(t)$ will get arbitrarily close to $\rho^{\mathrm{C}}$ in the long run; thus, we can find $\rho$ greater than but arbitrarily close to $\rho^{\mathrm{C}}$ such that $J(\rho \mid \sigma)>\rho^{\mathrm{C}}$. But we had earlier defined: $J\left(\rho^{\mathrm{C}} \mid \sigma\right) \equiv\left[\rho^{\mathrm{C}}-\chi\left(\rho^{\mathrm{C}} \mid \sigma\right)\right]$, where $\chi\left(\rho^{\mathrm{C}} \mid \sigma\right)>0$ is constant with time.

$\left.\left.B_{2}^{\dagger}(),. W_{2}^{\dagger}().\right) /\left[F\left(\rho R, B_{2}^{\dagger}(),. W_{2}^{\dagger}().\right)+F\left((1-\rho) R, B_{1}^{\dagger}(),. W_{1}^{\dagger}().\right)\right]\right\}$. Since $\rho \geq 0.5 \sigma /[1+\sigma] \geq \tilde{\rho}(\pi \mid \sigma) \forall \pi \in[0,1)$, we will use the Hobbesian equilibrium expressions for $B_{i}^{\dagger}($.$) and W_{i}^{\dagger}($.$) (provided in Proposition 2). Now,$ $\pi^{\dagger}(\rho \mid \sigma) \in[0,1)$ (see Proposition 4) is continuous in $\rho \in\left[0.5 \sigma /[1+\sigma], \rho^{+}(\sigma)\right)$. Replacing $\pi$ with $\pi^{\dagger}(\rho \mid \sigma)$ in the expressions for $B_{i}^{\dagger}($.$) and W_{i}^{\dagger}($.$) , it is immediate that B_{i}^{\dagger}\left(\sigma, \pi^{\dagger}(\rho \mid \sigma), R, \rho\right)$ and $W_{i}^{\dagger}\left(\sigma, \pi^{\dagger}(\rho \mid \sigma), R, \rho\right)$ are continuous in $\rho$. Now, $F($.) is defined to be continuous in its arguments and to be strictly positive; further, both $F\left(\rho R, B_{2}^{\dagger}\left(\sigma, \pi^{\dagger}(\rho \mid \sigma), R, \rho\right), W_{2}^{\dagger}\left(\sigma, \pi^{\dagger}(\rho \mid \sigma), R, \rho\right)\right)>0$ and $F\left((1-\rho) R, B_{1}^{\dagger}\left(\sigma, \pi^{\dagger}(\rho \mid \sigma), R, \rho\right), W_{1}^{\dagger}(\sigma\right.$, $\left.\left.\pi^{\dagger}(\rho \mid \sigma), R, \rho\right)\right)>0$ have all their arguments as being continuous in $\rho$, so each of them is continuous in $\rho$. Hence, with its denominator being strictly positive, $r^{\dagger}\left(\sigma, \pi^{\dagger}(\rho \mid \sigma), R, \rho\right) \equiv J(\rho \mid \sigma)$ is continuous in $\rho$. 
This implies that $J(\rho \mid \sigma)$ is discontinuous at $\rho^{\mathrm{C}}$. (If $J(\rho \mid \sigma)$ was continuous at $\rho^{\mathrm{C}}$ then it would be possible to find an interval, however small, around $\rho=\rho^{\mathrm{C}}$, such that $J(\rho \mid \sigma)$ calculated for all $\rho$ within the interval would be close to $J\left(\rho^{\mathrm{C}} \mid \sigma\right) \equiv\left[\rho^{\mathrm{C}}-\chi\left(\rho^{\mathrm{C}} \mid \sigma\right)\right]$.) We have obtained here a contradiction to the fact established before that $J(\rho \mid \sigma)$ is continuous for all $\rho \in\left[0.5 \sigma /[1+\sigma], \rho^{+}(\sigma)\right)$. So, our supposition that $\rho^{*}(t)>0.5 \sigma /[1+\sigma]$ for all $t \geq 1$ cannot be true. Hence, there will necessarily exist some finite generation such that $\rho^{*}(t) \leq 0.5 \sigma /[1+\sigma]$ for all $t$ after that generation. Then we obtain the result due to Proposition 8. This completes the proof of part $(i)$ of the Proposition. We next establish part (ii). Note that F-1.2 is a stricter condition than F-1.1. So, if $\rho(1)<\rho^{+}(\sigma)$ then the result follows immediately from part $(i)$. Henceforth, we consider the case when $\rho(1) \geq$ $\rho^{+}(\sigma)$. The proof is along a similar line as that of part $(i)$, and we only highlight the key features here. Note that if $\rho^{*}(T)<\rho^{+}(\sigma)$ in some generation $T$, then the result follows immediately from part (i). Next, note that even if property rights become perfect in some generation $t$ when $\rho^{*}(t) \geq \rho^{+}(\sigma)$, there will be a strict increase in resource inequality with: $\rho^{*}(t+1)<\rho^{*}(t)$, as imposed by Property F-1.2. If we assume that the equilibrium path converges (weakly) above $\rho^{+}(\sigma)$, then it would be possible for us to establish a contradiction. We would establish this contradiction by defining the same continuous function, $J(\rho \mid \sigma) \equiv r^{\dagger}\left(\sigma, \pi^{\dagger}(\rho \mid \sigma), R, \rho\right)$ as before, but now over the domain $\rho \in$ $\left[\rho^{+}(\sigma), 1 / 2\right)$, and then showing that the convergence of the equilibrium path above $\rho^{+}(\sigma)$ implies that the function is discontinuous at the assumed point of convergence. (Note that for all $\rho \in\left[\rho^{+}(\sigma), 1 / 2\right)$, it is the case that $\pi^{\dagger}(\rho \mid \sigma)=1$.) This contradiction will imply that $\rho^{*}(t)$ must fall strictly below $\rho^{+}(\sigma)$ in finite time; then part $(i)$ can be invoked to get the result.

[B] The proof for this part is analogous to that for [A], and we provide only the key features here. If in some generation $T, \rho^{*}(T) \in\left[0.5 \sigma /[1+\sigma], \rho^{+}(\sigma)\right)$ then $\rho^{*}(T+1)>\rho^{*}(T)$, due to Property F-2. Such an equilibrium path will have strictly decreasing resource inequality for all $t \geq T$, i.e., $\rho^{*}(t+1)>$ $\rho^{*}(t)$, as long as $\rho^{*}(t)<\rho^{+}(\sigma)$ (and if: $\rho^{*}(t) \geq \rho^{+}(\sigma)$ and $\pi^{*}(t)<1$ ). Then, after assuming that this path converges (strictly) below $\rho^{+}(\sigma)$ say to $\rho^{\#}$, we will be able to establish a contradiction by defining the same continuous function as before, $J(\rho \mid \sigma) \equiv r^{\dagger}\left(\sigma, \pi^{\dagger}(\rho \mid \sigma), R, \rho\right)$ over the domain $\left[0.5 \sigma /[1+\sigma], \rho^{+}(\sigma)\right)$, then also defining $J\left(\rho^{\#} \mid \sigma\right) \equiv\left[\rho^{\#}+(\right.$ a positive constant $\left.)\right]$ and showing that the convergence of the equilibrium path to $\rho^{\#}$ implies that the function is discontinuous at $\rho^{\#}$. Next, for the case when $\rho(1)<0.5 \sigma /[1+\sigma]$, we will assume that $\rho^{*}(t)$ gets captured strictly below $0.5 \sigma /[1+\sigma]$. But then eventually, anarchy would be established and community 2 would become a bandit. Then, using Property F-2 we will obtain the result that resource inequality is forever strictly decreasing. But we have also assumed that $\rho^{*}(t)$ gets captured strictly below $0.5 \sigma /[1+\sigma]$ which implies convergence of $\rho^{*}(t)$ (while strictly increasing) to some point (weakly) below $0.5 \sigma /[1+\sigma]$. By defining the same continuous function, $J(\rho \mid \sigma) \equiv r^{\dagger}\left(\sigma, \pi^{\dagger}(\rho \mid \sigma), R, \rho\right)$ as before, but now over the domain $\rho \in(0,0.5 \sigma /[1+\sigma]]$ (note the use of Banditry equilibrium expressions now, which still implies continuity of the function in its domain), we will be able to establish a contradiction by 
showing that the convergence of the equilibrium path below $0.5 \sigma /[1+\sigma]$ implies that the function is discontinuous at the assumed point of convergence. Hence, $\rho^{*}(t)$ cannot get captured strictly below $0.5 \sigma /[1+\sigma]$. This finally gives us the complete result (by using the second part of Proposition 8 ).

Proposition 9 is proved using the logic of the proofs of Propositions 8 and 10; we indicate the key steps. [A] Analogously as in Proposition 10, it can be proved that given any $\rho(1) \in(0,1 / 2),\left\{\rho^{*}(t)\right\}_{t=1}^{\infty}$ converges down to 0 . This implies that eventually resource inequality will persist at a sufficiently high level so that $\rho^{*}(t) \leq \rho^{-}(\sigma)$ for all $t$ large enough. Then, Proposition 8 implies the result.

[B] Analogously as in Proposition 10, it can be established that $\left\{\rho^{*}(t)\right\}_{t=1}^{\infty}$ will converge up to $1 / 2$. Hence, for all $t$ large we will have: $\rho^{*}(t) \geq \rho^{+}(\sigma)$. Then we get the result by using Proposition 8 .

[C] Given that $\boldsymbol{F}\left(R_{i}, B_{i}, W_{i}\right)=[1+\mu] R_{i}$, generational resource inequality does not change over time, i.e., $\rho^{*}(t)=\rho(1)$ for all $t$. Then the result follows immediately from Proposition 8 .

We now present a set of definitions and intermediate results that facilitate the proofs of Propositions $11-13$. When $\boldsymbol{F}$ (.) is a linear homogeneous $R L M$ with $v>0: r^{\dagger}(\sigma, \pi, R, \rho) \equiv\left\{\left[(1+\mu) \rho R+v \lambda B_{2}^{\dagger}()\right.\right.$. $\left.+v(1-\lambda) W_{2}^{\dagger}().\right] /\left[\left[(1+\mu)(1-\rho) R+v \lambda B_{1}^{\dagger}()+.v(1-\lambda) W_{1}^{\dagger}().\right]+\left[(1+\mu) \rho R+v \lambda B_{2}^{\dagger}()+.v(1-\right.\right.$ $\left.\left.\left.\lambda) W_{2}^{\dagger}().\right]\right]\right\}$. Dividing the numerator and the denominator by $(1+\mu) R$, and setting $y=v \lambda /(1+\mu)>0$, $z=v(1-\lambda) /(1+\mu)>0$, we have: $r^{\dagger}(\sigma, \pi, R, \rho) \equiv\left\{\left[\rho+y B_{2}^{\dagger}() / R+.z W_{2}^{\dagger}() / R.\right] /\left[1+y\left[B_{1}^{\dagger}()+.B_{2}^{\dagger}().\right] / R\right.\right.$ $\left.\left.+z\left[\mathrm{~W}_{1}^{\dagger}()+.W_{2}^{\dagger}().\right] / R\right]\right\}$. Note that $r^{\dagger}(\sigma, \pi, R, \rho) \in(0,1 / 2)$ and is continuous for all $\sigma \in[1 / 2,1), \pi \in[0,1]$, $R>0$ and $\rho \in(0,1 / 2)$; and that in any generational equilibrium we have $\left[B_{1}^{\dagger}()+.B_{2}^{\dagger}().\right]=\left[W_{1}^{\dagger}()+.W_{2}^{\dagger}().\right]$.

Given the linear homogeneous $R L M$, what level of resource inequality does generation $(t+1)$ inherit given that generation $t$ is in a Hobbesian equilibrium, or in a Banditry equilibrium? The linear homogeneous RLM satisfies properties that we summarize in Property LH-1 and Property LH-2 below, which help us answer the above questions. First, given $\sigma \in[0.5,1)$, we define the function:

$$
M(\pi \mid \sigma) \equiv[(1-\pi) / 2][1+\pi(1-\sigma)] /[1+\pi+\sigma(1-\pi)]>0, \text { for all } \pi \in[0,1) .
$$

At several places below, we denote $M(\pi \mid \sigma)$ more compactly as $M(\pi)$ and $\tilde{\rho}(\pi \mid \sigma)$ as $\tilde{\rho}(\pi)$.

Property LH-1: Take as given $\sigma \in[0.5,1)$ and the linear homogeneous $F\left(R_{i}, B_{i}, W_{i}\right)$ with $v>0$. Let $R>0, \pi \in[0,1)$ and $\rho \in[\widetilde{\rho}(\pi \mid \sigma), 1 / 2)$, then:

LH-1.1: $\left\{r^{\dagger}(\sigma, \pi, R, \rho)<(\right.$ resp. $>$; resp. $\left.=) \rho\right\}$ if and only if $\{\lambda>($ resp. $<$; resp. $=)[1+\pi(1-\sigma)] /[1$ $+\sigma+\pi(1-\sigma)]\}$.

LH-1.2: If $\lambda>[1-0.5 \sigma]$, then: $r^{\dagger}(\sigma, \pi, R, \rho)<\rho$.

LH-1.3: If $\lambda<1 /[1+\sigma]$, then: $r^{\dagger}(\sigma, \pi, R, \rho)>\rho$.

Proof of Property LH-1: Given $R>0, \pi \in[0,1)$ and $\rho \in[\widetilde{\rho}(\pi), 1 / 2)$, by using the relevant Hobbesian equilibrium expressions for $B_{i}^{\dagger}(\sigma, \pi, R, \rho)$ and $W_{i}^{\dagger}(\sigma, \pi, R, \rho)$ for $i=1,2$ we obtain: 
$r^{\dagger}(\sigma, \pi, R, \rho)=[\rho+y[\rho-\tilde{\rho}(\pi)]+z[\pi \rho+M(\pi)]] /[1+y[1-2 \tilde{\rho}(\pi)]+z[\pi+2 M(\pi)]]$.

Now, $r^{\dagger}(\sigma, \pi, R, \rho)<\rho \Leftrightarrow[\rho+y \rho-y \tilde{\rho}(\pi)+z \pi \rho+z M(\pi)] /[1+y-2 y \tilde{\rho}(\pi)+z \pi+2 z M(\pi)]<\rho$

$\Leftrightarrow z M(\pi)[1-2 \rho]<y \tilde{\rho}(\pi)[1-2 \rho] \Leftrightarrow z M(\pi)<y \tilde{\rho}(\pi) \Leftrightarrow z[(1-\pi) / 2][1+\pi(1-\sigma)] /[1+\pi+\sigma(1-\pi)]$ $<y \sigma[(1-\pi) / 2] /[1+\pi+\sigma(1-\pi)] \Leftrightarrow z[1+\pi(1-\sigma)]<y \sigma \Leftrightarrow \lambda>[1+\pi(1-\sigma)] /[1+\sigma+\pi(1-\sigma)]$.

The proofs of the other two parts of Property LH-1.1 follow the same line of argument. Also, Properties LH-1.2 and LH-1.3 follow immediately from Property LH-1.1 by setting $\pi=1$ and $\pi=0$ respectively in the second inequality of LH-1.1.

Property LH-2: Take as given $\sigma \in[0.5,1)$ and the linear homogeneous $F\left(R_{i}, B_{i}, W_{i}\right)$ with $v>0$. Let $R>0, \pi \in[0,1)$ and $\rho \in(0, \tilde{\rho}(\pi \mid \sigma)]$, then:

LH-2.1: $r^{\dagger}(\sigma, \pi, R, \rho)$ is strictly increasing in $\rho \in(0, \tilde{\rho}(\pi \mid \sigma)]$.

LH-2.2: If $\lambda>[1-0.5 \sigma]$, then: $r^{\dagger}(\sigma, \pi, R, \rho) \in(0, \tilde{\rho}(\pi \mid \sigma))$.

LH-2.3: If $\lambda<1 /[1+\sigma]$, then: $r^{\dagger}(\sigma, \pi=0, R, \rho)>\rho, \forall \rho \in(0, \tilde{\rho}(\pi=0 \mid \sigma)]$.

Proof of Property LH-2: Given $R>0, \pi \in[0,1)$ and $\rho \in(0, \tilde{\rho}(\pi)]$, by using the relevant Banditry equilibrium expressions for $B_{i}^{\dagger}(\sigma, \pi, R, \rho)$ and $W_{i}^{\dagger}(\sigma, \pi, R, \rho)$ for $i=1,2$, we get: $r^{\dagger}(\sigma, \pi, R, \rho)=$ $\left\{\rho+z[\pi+\sigma(1-\pi)][4 \rho \tilde{\rho}(\pi)]^{1 / 2}+z(1-\sigma)(1-\pi) / 2-z \sigma \rho(1-\pi)\right\} /\left\{1+(y+z)\left[1-[4 \rho \tilde{\rho}(\pi)]^{1 / 2}\right]\right\}$.

It can be shown through straightforward algebra that the numerator given above is strictly increasing while the denominator is strictly decreasing in $\rho \in(0, \tilde{\rho}(\pi)]$. Hence, $r^{\dagger}(\sigma, \pi, R, \rho)$ is strictly increasing in $\rho \in(0, \tilde{\rho}(\pi)]$. This establishes Property LH-2.1. Property LH-1.2 established that if $\lambda>[1-0.5 \sigma]$, then for any $\pi<1$ we have: $r^{\dagger}(\sigma, \pi, R, \rho=\tilde{\rho}(\pi))<\tilde{\rho}(\pi)$. This fact, combined with Property LH-2.1, implies: when $\lambda>[1-0.5 \sigma]$ then given any $\pi<1, r^{\dagger}(\sigma, \pi, R, \rho) \in(0, \tilde{\rho}(\pi))$ for all $\rho \in(0, \tilde{\rho}(\pi)]$. This establishes Property LH-2.2. Next, it can be easily seen (by setting $\rho=0)$ that $r^{\dagger}(\sigma, \pi=0, R, \rho)>\rho$ for $\rho$ close to 0 . Finally, for the case that $\lambda<1 /[1+\sigma]$, it can be shown through straightforward algebra that $r^{\dagger}(\sigma, \pi=0, R, \rho)=\rho$ has no solution for $\rho \in(0, \tilde{\rho}(\pi)]$. Since $r^{\dagger}(\sigma, \pi=0, R, \rho)$ is a continuous function in $\rho$, this implies Property LH-2.3.

\section{ProOF OF PROPOSITION 11:}

Let $\sigma \in[1 / 2,1), \pi \in[0,1], R>0$ and $\rho \in(0,1 / 2)$.

[A] For the linear homogeneous $R L M$ with $\lambda \in([1-0.5 \sigma], 1)$ and $v>0$, given any $\pi \in[0,1)$ : if $\rho \in$ $[\tilde{\rho}(\pi), 1 / 2)$ then $r^{\dagger}(\sigma, \pi, R, \rho)<\rho$ (by Property LH-1.2), while if $\rho<\tilde{\rho}(\pi)$ then $r^{\dagger}(\sigma, \pi, R, \rho)<\tilde{\rho}(\pi)$ (by Property LH-2.2). So, if $\lambda \in([1-0.5 \sigma], 1)$ then the linear homogeneous $R L M$ satisfies Property F-1.1.

[B] For the linear homogeneous $R L M$ with $\lambda \in(0,[1 /(1+\sigma)])$ and $v>0$, given any $\pi \in[0,1)$ : if $\rho \in$ $[\tilde{\rho}(\pi), 1 / 2)$ then $r^{\dagger}(\sigma, \pi, R, \rho)>\rho$ (by Property LH-1.3), while if $\rho<\tilde{\rho}(\pi=0)$ then $r^{\dagger}(\sigma, \pi=0, R, \rho)>\rho$ (by Property LH-2.3). Finally, $r^{\dagger}(\sigma, \pi=1, R, \rho)=\rho$. Hence, if $\lambda \in(0,[1 /(1+\sigma)])$, then the linear homogeneous $R L M$ satisfies Property F-2. 


\section{ProOF OF PROPOSITION 12:}

[A] Note that if $\rho^{*}(t) \geq \rho^{+}(\sigma)$ in some generation $t$, then $\pi^{*}(t)=\min \left\{\left[\pi^{*}(t-1)+\eta\right], 1\right\}$. We are given here that $\rho(1)>\rho^{+}(\sigma)$, so if $\eta \geq[1-\pi(0)]$ then we will get: $\pi^{*}(1)=1$. Then, given the linear homogeneous $R L M$, we will necessarily obtain: $\rho^{*}(t)=\rho(1)$ and $\pi^{*}(t)=1$ for all $t>1$. So, for sufficiently large values of $\eta$, the equilibrium path will attain perfect property rights.

Now, since $\lambda \in([1-0.5 \sigma], 1)$, therefore by Property LH-1.2, in any generation $t$ in which $\pi^{*}(t)<1$ and $\rho^{*}(t)>\tilde{\rho}\left(\pi^{*}(t)\right)$ it will be the case that: $\rho^{*}(t+1)<\rho^{*}(t)$. Hence, if in generation $t$ we have $\left[\pi^{*}(t-1)+\eta\right]<1$ and $\rho^{*}(t) \geq \rho^{+}(\sigma)$, then: $1>\pi^{*}(t)>\pi^{*}(t-1)$, while $\rho^{*}(t+1)<\rho^{*}(t)$. So, given $\rho(1)$ $>\rho^{+}(\sigma)$ and $\pi(0) \in[0,1)$, for small values of $\eta$ both property rights and resource inequality will initially increase. We will show next that for sufficiently small but non-zero values of $\eta$, $\rho^{*}(t)$ will fall strictly below $\rho^{+}(\sigma)$ in finite time (before $\pi^{*}(t)$ is able to attain the value of 1 ). Then, by invoking Proposition 8 we will obtain the result that the equilibrium path devolves to anarchy.

Recall that $\rho^{*}(t+1)=r^{\dagger}\left(\sigma, \pi^{*}(t), R^{*}(t), \rho^{*}(t)\right)$. Given $R>0, \pi \in[0,1), \rho \in[\tilde{\rho}(\pi), 1 / 2)$ and $\lambda \in([1-0.5 \sigma]$, 1), we have: $\left[\rho-r^{\dagger}(\sigma, \pi, R, \rho)\right]=[1-2 \rho][y \tilde{\rho}(\pi)-z M(\pi)] /[1+y[1-2 \tilde{\rho}(\pi)]+z[\pi+2 M(\pi)]]>0$.

It is easily seen that $\left[\rho-r^{\dagger}(\sigma, \pi, R, \rho)\right]$ strictly decreases in $\rho$, it can also be algebraically established that it strictly decreases in $\pi$. The implication of this is that both for lower values of $\pi$ and for lower values of $\rho$, the increase in resource inequality will be larger. If we can show that, starting at $\rho(1)>$ $\rho^{+}(\sigma), \rho^{*}(t)$ will fall below $\rho^{+}(\sigma)$ for one non-zero value of $\eta$, then $\rho^{*}(t)$ will also necessarily fall below $\rho^{+}(\sigma)$ for all lower values of $\eta$ (since when $\eta$ is smaller, $\left[\pi^{*}(1)-\pi(0)\right]$ will be smaller and so $\left[\rho(1)-\rho^{*}(2)\right]$ will be larger, both of which lead to lower values of $\pi^{*}(1)$ and $\rho^{*}(2)$ - as compared to the case when $\eta$ is larger - and then we will also get lower values of $\pi^{*}(2)$ and $\rho^{*}(3)$, and so on). We will next establish that there necessarily exists one non-zero value of $\eta$ given which $\rho^{*}(t)$ will fall below $\rho^{+}(\sigma)$ in finite time. Pick any value of property rights $\pi^{\prime} \in(\pi(0), 1)$. Now, consider positive numbers $\eta$ and positive integers $T$ which satisfy the following two conditions: $(i)[\pi(0)+$ $\eta .(T+1)]<\pi^{\prime}$, and $(i i)\left[\rho(1)-\rho^{+}(\sigma)\right]<T .\left[\rho(1)-r^{\dagger}\left(\sigma, \pi^{\prime}, R, \rho(1)\right)\right]$. It is easy to see that for large enough $T$ the second condition will be satisfied, and then given this $T$ we can find a small enough $\eta$ such that the first condition is also satisfied. Given such an $\eta$, it is easy to see that $\rho^{*}(t)$ will fall below $\rho^{+}(\sigma)$ in $T$ or fewer generations while $\pi^{*}(t)$ will not even be able to attain the value $\pi^{\prime}<1$ in that interval. Hence, given any $\pi(0) \in[0,1)$ there exists $\eta^{0} \in(0,1)$ such that if $\eta<\eta^{0}$, the equilibrium path $\left\{\pi^{*}(t), \rho^{*}(t)\right\}_{t=1}^{\infty}$ devolves to anarchy.

[B] Given the linear homogeneous $R L M$ with $\lambda \in([1 /(1+\sigma)],[1-0.5 \sigma])$, we can determine a critical value of property rights, $\pi^{c}(\lambda \mid \sigma) \in(0,1)$, by using Property LH-1.1 and setting: $\lambda=\left[1+\pi^{c}(\lambda \mid\right.$ $\sigma)(1-\sigma)] /\left[1+\sigma+\pi^{c}(\lambda \mid \sigma)(1-\sigma)\right] \Rightarrow \pi^{c}(\lambda \mid \sigma) \equiv[\lambda(1+\sigma)-1] /[(1-\sigma)(1-\lambda)]$ for all $\lambda \in([1 /(1+\sigma)],[1-$ $0.5 \sigma])$. We also define $\rho^{c}(\lambda \mid \sigma) \in\left(\rho^{-}(\sigma), \rho^{+}(\sigma)\right)$ by setting: $\pi^{\dagger}\left(\rho^{c}(\lambda \mid \sigma) \mid \sigma\right)=\pi^{c}(\lambda \mid \sigma)$ for all $\lambda \in$ $([1 /(1+\sigma)],[1-0.5 \sigma])$. Now, we obtain an important property satisfied by the equilibrium path 
when $\lambda \in([1 /(1+\sigma)],[1-0.5 \sigma])$ (which basically follows from a re-arrangement of LH-1.1) In a generation $t$ in which $\pi^{*}(t)<1$ and $\rho^{*}(t) \in\left[\tilde{\rho}\left(\pi^{*}(t) \mid \sigma\right), 1 / 2\right)$, we will have: $\rho^{*}(t+1)<\rho^{*}(t)$ if $\pi^{*}(t)<\pi^{c}(\lambda \mid \sigma)$, while; $\rho^{*}(t+1)>\rho^{*}(t)$ if $\pi^{*}(t)>\pi^{c}(\lambda \mid \sigma)$, and; $\rho^{*}(t+1)=\rho^{*}(t)$ if $\pi^{*}(t)=\pi^{c}(\lambda \mid \sigma)$. Note that $[0.5 \sigma /(1+\sigma)] \geq \tilde{\rho}(\pi \mid \sigma)$ for all $\pi<1$. Now, consider the case when $\rho(1) \in[0.5 \sigma /(1+\sigma)$, $\left.\rho^{c}(\lambda \mid \sigma)\right)$ and $\pi(0) \leq \pi^{c}(\lambda \mid \sigma)$. Since $\pi^{\dagger}(\rho(1) \mid \sigma)<\pi^{\dagger}\left(\rho^{c}(\lambda \mid \sigma) \mid \sigma\right)=\pi^{c}(\lambda \mid \sigma)$, we get: $\pi^{*}(1)<\pi^{c}(\lambda \mid$ $\sigma)$. Then, using the property established above we get: $\rho^{*}(2)<\rho(1)$. Then, since $\pi^{\dagger}\left(\rho^{*}(2) \mid \sigma\right)<$ $\pi^{c}(\lambda \mid \sigma)$ (and since $\pi^{*}(1)<\pi^{c}(\lambda \mid \sigma)$ ), we get: $\pi^{*}(2)<\pi^{c}(\lambda \mid \sigma)$. If $\rho^{*}(2) \geq 0.5 \sigma /(1+\sigma)$, then we will get: $\rho^{*}(3)<\rho^{*}(2)$, and so on. It can be shown with the technique used in the proof of Proposition $8[\mathrm{~A}](i)$ that such an equilibrium path cannot converge weakly above $0.5 \sigma /(1+\sigma)$. So, there exists a finite generation $T$ in which $\rho^{*}(T) \leq 0.5 \sigma /(1+\sigma)$ and $\pi^{*}(T)<\pi^{c}(\lambda \mid \sigma)$. Then, by using Property LH2.1 and the property established above we obtain that $\rho^{*}(T+1) \leq 0.5 \sigma /(1+\sigma)$ and so $\pi^{*}(T+1) \leq \pi^{*}(T)$ $\left(<\pi^{c}(\lambda \mid \sigma)\right)$. Going on like this, we get that: $\rho^{*}(t) \leq 0.5 \sigma /(1+\sigma)\left(<\rho^{-}(\sigma)\right)$ for all $t \geq T$. Then, by invoking Proposition 8 we obtain that the equilibrium path devolves to anarchy. Note that when $\rho(1)<0.5 \sigma /(1+\sigma)$ and $\pi(0) \leq \pi^{c}(\lambda \mid \sigma)$, the equilibrium path will devolve to anarchy.

Finally, consider the case when $\rho(1)<\rho^{c}(\lambda \mid \sigma)$ and $\pi(0) \in\left(\pi^{c}(\lambda \mid \sigma)\right.$, 1). If $\varepsilon \geq\left[\pi(0)-\pi^{\dagger}(\rho(1) \mid \sigma)\right]$, then $\pi^{*}(1)=\pi^{\dagger}(\rho(1) \mid \sigma)<\pi^{c}(\lambda \mid \sigma)$. Then, as shown above, there is devolution to anarchy. ${ }^{70}$

\section{PROOF OF PROPOSITION 13:}

Given that the linear homogeneous $R L M$ (with $v>0$ ) induces an equilibrium path that devolves to anarchy, it cannot be the case that $\lambda \in(0,[1 /(1+\sigma)])$ (by Proposition 11[B]). So below we consider two cases: (i) $\lambda \in([1-0.5 \sigma], 1)$, and (ii) $\lambda \in([1 /(1+\sigma)],[1-0.5 \sigma]]$.

In the case where $\lambda \in([1-0.5 \sigma], 1)$, since the equilibrium path devolves to anarchy (by Proposition 11[A]), we will obtain $\rho^{*}(t) \leq \rho^{-}(\sigma)$ for all $t$ beyond some finite generation, say $T$. This follows from Proposition 8. Consequently, the hypotheses of Proposition 10[A](i) $-\{\boldsymbol{F}($.$) is homogeneous$ and satisfies Property F-1.1\} and $\left\{\rho(1)<\rho^{+}(\sigma)\right\}$ - are also satisfied (after re-naming $T$ as the first generation). So, as we established in the proof of Proposition 10[A](i), eventually resource inequality persists at a sufficiently high level such that $\rho^{*}(t) \leq 0.5 \sigma /[1+\sigma]$ for all $t$ beyond some finite generation. Given that $\rho^{*}(t) \leq 0.5 \sigma /[1+\sigma]$ and $\pi^{*}(t)=0$ for all $t$ large, and noting that $\tilde{\rho}(\pi=0)$ $=0.5 \sigma /[1+\sigma]$, the communities will persist in the Banditry equilibrium beyond a finite generation.

Next, our goal is to show that starting at $\rho^{*}(\tau) \leq 0.5 \sigma /[1+\sigma]$ and $\pi^{*}(\tau)=0$ in generation $\tau, \rho^{*}(t)$ converges to a specific value strictly less than $0.5 \sigma /[1+\sigma]$. Given any $t \geq \tau$, the resource inequality

\footnotetext{
${ }^{70}$ Recognize that for sufficiently small values of $\varepsilon$, we would get here: $\pi^{*}(1)=[\pi(0)-\varepsilon]>\pi^{c}(\lambda \mid \sigma)$. Then, we would obtain: $\rho^{*}(2)>\rho(1)$ (by using the equilibrium property established before). As this path evolves, it is possible that in some finite generation $\tau$ we have: $\rho^{*}(\tau)>\rho^{c}(\lambda \mid \sigma)$ and $\pi^{*}(\tau)>\pi^{c}(\lambda \mid \sigma)$. Thereafter, it can be shown that this path would attain perfect property rights.
} 
in generation $(t+1)$ is given by $\rho^{*}(t+1)=r^{\dagger}\left(\sigma, \pi^{*}(t)=0, R^{*}(t), \rho^{*}(t)\right)$, which is independent of $R^{*}(t)$ due to the homogeneous $R L M$. In order to establish convergence of the time path, we will next inspect the function $r^{\dagger}(\sigma, \pi=0, R, \rho)$ for $\rho \in(0, \tilde{\rho}(\pi=0)]$, given any $\sigma \in[1 / 2,1)$ and $R>0$. Note that the function is independent of $R$. Now, using Property LH-2.1 we have that: $r^{\dagger}(\sigma, \pi=0, R, \rho)$ is strictly increasing in $\rho \in(0, \tilde{\rho}(\pi=0)]$; further, by Property LH-2.2 we have that: $r^{\dagger}(\sigma, \pi=0, R, \rho=$ $\tilde{\rho}(\pi=0))<\tilde{\rho}(\pi=0)$. It can also be seen easily that $r^{\dagger}(\sigma, \pi=0, R, \rho)>\rho$ for $\rho$ close to 0 . Hence, since $r^{\dagger}(\sigma, \pi=0, R, \rho)$ is continuous in $\rho \in(0, \tilde{\rho}(\pi=0)]$, it must equal $\rho$ at least once in $\rho \in(0, \tilde{\rho}(\pi=0))$. It can be shown through straightforward algebra that $r^{\dagger}(\sigma, \pi=0, R, \rho)=\rho$ has, in fact, a unique solution for $\rho \in\left(0, \tilde{\rho}(\pi=0)\right.$ ). Let us denote it by $\rho^{\infty}$ (which happens to solely be a function of $\lambda$ and $\sigma$ ). The properties of $r^{\dagger}(\sigma, \pi=0, R, \rho)$ established above imply that if $\rho^{*}(t)>\rho^{\infty}$ in some $t \geq \tau$ then $\rho^{*}(t+1)=$ $r^{\dagger}\left(\sigma, \pi^{*}(t)=0, R^{*}(t), \rho^{*}(t)\right) \in\left(\rho^{\infty}, \rho^{*}(t)\right)$, while if $\rho^{*}(t)<\rho^{\infty}$ in some $t \geq \tau$ then $\rho^{*}(t+1) \in\left(\rho^{*}(t)\right.$, $\left.\rho^{\infty}\right)$. Hence, there are three possible cases:

(a) If $\rho^{*}(\tau)>\rho^{\infty}$, then $\rho^{*}(\tau)>\rho^{*}(\tau+1)>\ldots>\rho^{\infty}$.

(b) If $\rho^{*}(\tau)<\rho^{\infty}$, then $\rho^{*}(\tau)<\rho^{*}(\tau+1)<\ldots<\rho^{\infty}$.

(c) If $\rho^{*}(\tau)=\rho^{\infty}$, then $\rho^{*}(\tau)=\rho^{*}(\tau+1)=\ldots=\rho^{\infty}$. (Note that this is likely to be a non-generic case.)

It is straightforward to show in cases $(a)$ and $(b)$ that the equilibrium path must converge to $\rho^{\infty}$. We can establish this by assuming to the contrary that the path converges to an alternative point, and then establishing that the continuous function of $\rho, r^{\dagger}(\sigma, \pi=0, R, \rho)$ is discontinuous at the posited alternative point of convergence. This contradiction provides the result.

In the case where $\lambda \in([1 /(1+\sigma)],[1-0.5 \sigma]]$, since the equilibrium path devolves to anarchy, using Proposition 8 we have that $\rho^{*}(t) \leq \rho^{-}(\sigma)$ for all $t$ beyond some finite generation. Then, by using the same technique that we used in the proof of Proposition 10[A](i), we can establish that resource inequality eventually persists at a sufficiently high level such that $\rho^{*}(t) \leq 0.5 \sigma /[1+\sigma]$ after some finite generation. Finally, using the same logic as in the case above, we can complete the proof that the equilibrium path converges to $\rho^{\infty} \in(0, \tilde{\rho}(\pi=0))$.

\section{PROOF OF PROPOSITION 14:}

Since $\zeta^{\prime \prime}$ induces an equilibrium path that attains perfect property rights, it is eventually the case that $\left.B_{i}^{*}\left(t \mid \zeta^{\prime \prime}\right) \approx W_{i}^{*}\left(t \mid \zeta^{\prime \prime}\right) \approx R_{i}^{*}\left(t \mid \zeta^{\prime \prime}\right)\right]$ for $i=1,2$ and large $t$. Thus, the rate of growth in aggregate resource will eventually become arbitrarily close to $\left[\mu\left(\zeta^{\prime \prime}\right)+v\left(\zeta^{\prime \prime}\right)\right]$ with: $R^{*}\left(t+1 \mid \zeta^{\prime \prime}\right) \approx\left[1+\mu\left(\zeta^{\prime \prime}\right)+\right.$ $\left.v\left(\zeta^{\prime \prime}\right)\right] \cdot\left[R^{*}\left(t \mid \zeta^{\prime \prime}\right)\right]$. Further, Proposition 8 establishes that if an equilibrium path exhibits attainment of perfect property rights then it also exhibits attainment of low resource inequality. So eventually $\left[R_{2}^{*}\left(t \mid \zeta^{\prime \prime}\right) / R^{*}\left(t \mid \zeta^{\prime \prime}\right)\right] \geq \rho^{+}\left(\sigma\left(\zeta^{\prime \prime}\right)\right)$ (or, it will be arbitrarily close to $\left.\rho^{+}\left(\sigma\left(\zeta^{\prime \prime}\right)\right)\right)$, while $\left[R_{1} *\left(t \mid \zeta^{\prime \prime}\right) / R^{*}(t\right.$ $\left.\left.\mid \zeta^{\prime \prime}\right)\right] \geq 0.5$. So, for large enough $t^{\prime}$ s, $\left[W_{i}^{*}\left(t \mid \zeta^{\prime \prime}\right) / R^{*}\left(t \mid \zeta^{\prime \prime}\right)\right]=\left[W_{i}^{*}\left(t \mid \zeta^{\prime \prime}\right) / R_{i}^{*}\left(t \mid \zeta^{\prime \prime}\right)\right] \times\left[R_{i}^{*}\left(t \mid \zeta^{\prime \prime}\right) / R^{*}(t\right.$ $\left.\left.\mid \zeta^{\prime \prime}\right)\right]$ will be no less than a positive time-invariant constant that is strictly between zero and one. 
Next, vector $\zeta^{\prime}$ induces an equilibrium path that devolves to anarchy. Proposition 13 establishes that with the linear homogeneous $R L M$, such a path will converge to $\rho^{\infty}\left(\zeta^{\prime}\right) \in\left(0,\left[0.5 \sigma\left(\zeta^{\prime}\right) /\left(1+\sigma\left(\zeta^{\prime}\right)\right)\right]\right)$ and community 2 will become a bandit. ${ }^{71}$ So, eventually $G_{1}{ }^{*}\left(t \mid \zeta^{\prime}\right)>G_{2}{ }^{*}\left(t \mid \zeta^{\prime}\right)=R_{2}{ }^{*}\left(t \mid \zeta^{\prime}\right) \approx$ $\rho^{\infty}\left(\zeta^{\prime}\right) \cdot\left[R^{*}\left(t \mid \zeta^{\prime}\right)\right]$. Hence, eventually $\left[B_{1}^{*}\left(t \mid \zeta^{\prime}\right)+B_{2}^{*}\left(t \mid \zeta^{\prime}\right)\right]<\left[1-2 \rho^{\infty}\left(\zeta^{\prime}\right)\right] \cdot\left[R^{*}\left(t \mid \zeta^{\prime}\right)\right]$. As a result, for all $t$ large enough: $R^{*}\left(t+1 \mid \zeta^{\prime}\right)<\left[1+\mu\left(\zeta^{\prime}\right)+v\left(\zeta^{\prime}\right) .\left\{1-2 \rho^{\infty}\left(\zeta^{\prime}\right)\right\}\right] .\left[R^{*}\left(t \mid \zeta^{\prime}\right)\right]$ implying that the rate of growth of aggregate resources is eventually bounded below $\left[\mu\left(\zeta^{\prime}\right)+v\left(\zeta^{\prime}\right)\right]$.

We thus conclude that there exists some finite generation $\tau$ such that for all $t>\tau: R^{*}\left(t \mid \zeta^{\prime \prime}\right) \approx$ $\left[1+\mu\left(\zeta^{\prime \prime}\right)+v\left(\zeta^{\prime \prime}\right)\right]^{(t-\tau)} \cdot\left[R^{*}\left(\tau \mid \zeta^{\prime \prime}\right)\right]$, while $R^{*}\left(t \mid \zeta^{\prime}\right)<\left[1+\mu\left(\zeta^{\prime}\right)+v\left(\zeta^{\prime}\right) .\left\{1-2 \rho^{\infty}\left(\zeta^{\prime}\right)\right\}\right]^{(t-\tau)} \cdot\left[R^{*}\left(\tau \mid \zeta^{\prime}\right)\right]$.

We also have: $\left[\mu\left(\zeta^{\prime \prime}\right)+v\left(\zeta^{\prime \prime}\right)\right] \geq\left[\mu\left(\zeta^{\prime}\right)+v\left(\zeta^{\prime}\right)\right]>\left[\mu\left(\zeta^{\prime}\right)+v\left(\zeta^{\prime}\right) .\left(1-2 \rho^{\infty}\left(\zeta^{\prime}\right)\right)\right]$, so eventually we will have: $R^{*}\left(t \mid \zeta^{\prime \prime}\right)>R^{*}\left(t \mid \zeta^{\prime}\right)$. In fact, any (time-invariant and positive) fraction of $R^{*}\left(t \mid \zeta^{\prime \prime}\right)$ will eventually be larger than the whole of $\left[R^{*}\left(t \mid \zeta^{\prime}\right)\right] .{ }^{72}$ So, there exists a finite $T$ such that for all $t>T$ : $\left[W_{i}^{*}\left(t \mid \zeta^{\prime \prime}\right)\right]>\left[W_{i}^{*}\left(t \mid \zeta^{\prime}\right)\right]$ for $i=1,2$. Lastly, Section 3.2.3 presents an example in which there exist multiple generations $t \leq T$ such that $\left[W_{i}^{*}\left(t \mid \zeta^{\prime \prime}\right)\right]<\left[W_{i}^{*}\left(t \mid \zeta^{\prime}\right)\right]$.

\section{PROOF OF PROPOSITION 15:}

Consider first the equilibrium path without the social planner's intervention. Since it devolves to anarchy and given that there is no institutional inertia, it must be the case that $\rho^{*}(t \mid \zeta)<\rho^{+}(\sigma)$ for all $t \geq 1$. [If it was that $\rho^{*}\left(t^{\prime} \mid \zeta\right) \geq \rho^{+}(\sigma)$ in some generation $t^{\prime}$ then we would have: $\pi^{*}\left(t^{\prime} \mid \zeta\right)=1$ for all $t$ $\geq t^{\prime}$ and hence anarchy would never be established.] Note that $\rho^{*}(t \mid \zeta)<\rho^{+}(\sigma)$ for all $t \geq 1$ implies that $\pi^{*}(t \mid \zeta)<1$ for all $t \geq 1$. Hence, in every generation $t \geq 1$ we have the following: $W_{1}^{*}(t \mid \zeta)<$ $R_{1} *(t \mid \zeta)$ and $\left[W_{1}^{*}(t \mid \zeta)+W_{2} *(t \mid \zeta)\right]=\left[B_{1} *(t \mid \zeta)+B_{2} *(t \mid \zeta)\right]<\left[R_{1} *(t \mid \zeta)+R_{2} *(t \mid \zeta)\right]$, and; $R_{1} *(t+1 \mid \zeta)$ $<[1+\mu+v] R_{1} *(t \mid \zeta)$ and $\left[R_{1} *(t+1 \mid \zeta)+R_{2} *(t+1 \mid \zeta)\right]<[1+\mu+v]\left[R_{1} *(t \mid \zeta)+R_{2} *(t \mid \zeta)\right]$.

In contrast, given an intervention by the planner in some generation $t^{\prime \prime}$ (that sets $\pi(t)=1$ exogenously for all $\left.t \geq t^{\prime \prime}\right)$, in any generation $t \geq t^{\prime \prime}$ we have: $W_{1}{ }^{[1]}(t \mid \zeta)=B_{1}{ }^{[1]}(t \mid \zeta)=R_{1}{ }^{[1]}(t \mid \zeta)$ and $\left[W_{1}{ }^{[1]}(t \mid \zeta)+W_{2}{ }^{[1]}(t \mid \zeta)\right]=\left[B_{1}{ }^{[1]}(t \mid \zeta)+B_{2}{ }^{[1]}(t \mid \zeta)\right]=\left[R_{1}{ }^{[1]}(t \mid \zeta)+R_{2}{ }^{[1]}(t \mid \zeta)\right]$, and; $R_{1}{ }^{[1]}(t+1 \mid \zeta)=$ $[1+\mu+v] R_{1}^{[1]}(t \mid \zeta)$ and $\left[R_{1}^{[1]}(t+1 \mid \zeta)+R_{2}^{[1]}(t+1 \mid \zeta)\right]=[1+\mu+v]\left[R_{1}{ }^{[1]}(t \mid \zeta)+R_{2}^{[1]}(t \mid \zeta)\right]$.

The above facts immediately imply that given an intervention by the social planner in generation $t^{[1]}$, we have: $W_{1}^{[1]}(t \mid \zeta)>W_{1} *(t \mid \zeta)$ and $\left\{\left[W_{1}^{[1]}(t \mid \zeta)\right]+\left[W_{2}{ }^{[1]}(t \mid \zeta)\right]\right\}>\left\{\left[W_{1}{ }^{*}(t \mid \zeta)\right]+\left[W_{2}{ }^{*}(t \mid \zeta)\right]\right\}$, for all $t$ $\geq t^{[1]}$. But note that since $\pi^{*}\left(t^{[1]} \mid \zeta\right)<1$, therefore $\left[W_{2}^{*}\left(t^{[1]} \mid \zeta\right)\right]>\left[W_{2}^{[1]}\left(t^{[1]} \mid \zeta\right)\right]$. As was established in Proposition 14, eventually the rate of growth in aggregate resource in the case when the

\footnotetext{
${ }^{71}$ In the non-generic case when $\lambda\left(\zeta^{\prime}\right)=1 /\left[1+\sigma\left(\zeta^{\prime}\right)\right]$ and the equilibrium path 'devolves to anarchy', the path will converge to some $r^{\infty}\left(\zeta^{\prime}\right) \in\left[0.5 \sigma\left(\zeta^{\prime}\right) /\left[1+\sigma\left(\zeta^{\prime}\right)\right], \rho^{-}\left(\sigma\left(\zeta^{\prime}\right)\right)\right]$. The analysis for this non-generic case will be very similar to the analysis provided below, and the result will still hold.

72 In fact, this will hold even if $\left[\mu\left(\zeta^{\prime}\right)+v\left(\zeta^{\prime}\right) .\left\{1-2 \rho^{\infty}\left(\zeta^{\prime}\right)\right\}\right]<\left[\mu\left(\zeta^{\prime \prime}\right)+v\left(\zeta^{\prime \prime}\right)\right] \Leftrightarrow\left[\mu\left(\zeta^{\prime}\right)+v\left(\zeta^{\prime}\right)\right]<\left[\mu\left(\zeta^{\prime \prime}\right)+\right.$ $\left.v\left(\zeta^{\prime \prime}\right)+2 v\left(\zeta^{\prime}\right) \cdot \rho^{\infty}\left(\zeta^{\prime}\right)\right]$
} 
equilibrium path devolves to anarchy is bounded below $[\mu+v]$. In contrast, the rate of growth in aggregate resource when the social planner acts is exactly $[\mu+v]$ from the time of intervention. Further, in the case of intervention, the poorer community possesses a generational resource endowment that is a strictly positive and constant fraction of aggregate resource in each generation (which is easily deduced from the resource inequality that exists in the generation in which the social planner acted). Since property rights are perpetually set at 1 after intervention, the poorer community consumes its own resource in every generation. So, the rate of growth in the poorer community's consumption is exactly $[\mu+v]$. So there exists a finite $T \geq t^{[1]}$ such that for all $\tau>T$ : $\left[W_{2}^{[1]}(\tau \mid \zeta)\right]>\left[R^{*}(\tau \mid \zeta)\right]>\left[W_{2} *(\tau \mid \zeta)\right]$. Finally, we presented an example after Proposition 15 in which there exist multiple generations $t$ greater than but close to $t^{[1]}$ with $\left[W_{2}{ }^{*}(t \mid \zeta)\right]>\left[W_{2}{ }^{[1]}(t \mid \zeta)\right]$.

\section{References}

Alchian, A. and H. Demsetz. (1973) “The Property Right Paradigm," Journal of Economic History 33, 16-27.

Anbarci, N., S. Skaperdas, and C. Syropoulos. (2002) "Comparing Bargaining Solutions in the Shadow of Conflict," Journal of Economic Theory 106, 1- 16.

Besley, T. and M. Ghatak. (2010) "Property Rights and Economic Development," in D. Rodrik and M. Rosenzweig (eds.) Handbook of Development Economics, North Holland: Amsterdam.

Besley, T. and T, Persson. (2009) "The Origins of State Capacity: Property Rights, Taxation and Politics," American Economic Review 99, 1218-44.

Bevia, C. and L. Corchon. (2010) "Peace Agreements without Commitment," Games and Economic Behaviour 68, 469-87.

Boyce, J. and D. Bruner. (2012) "Property Rights out of Anarchy? The Demsetz Hypothesis in a Game of Conflict," Economics and Governance 13, 95-120.

Demsetz, H. (1967) “Towards a Theory of Property Rights,” American Economic Review 57, 34759.

Dixit, A. (2004) Lawlessness and Economics: Alternative Modes of Governance, Princeton University Press: Princeton.

Ensminger, J. (1992) Making a Market: The Institutional Transformation of an African Society, Cambridge University Press: Cambridge.

Eswaran, M. \& H. Neary. (2014) "An Economic Theory of the Evolutionary Emergence of Property Rights," American Economic Journal: Microeconomics 6, 203-26.

Garfinkel, M. and S. Skaperdas. (2000) "Conflict without Misperceptions or Incomplete Information," Journal of Conflict Resolution 44, 793 - 807.

Garfinkel, M. and S. Skaperdas. (2007) "Economics of Conflict: An Overview," in T. Sandler and K. Hartley (eds.), Handbook of Defense Economics II, 649-709, North Holland: Amsterdam.

Genicot, G. and S. Skaperdas. (2002) "Investing in Conflict Management," Journal of Conflict 
Resolution 140, 154-70.

Goel, B. (2017) "Contest Theory and its Applications to Development and Political Economy," under preparation, Indian Institute of Management Calcutta.

Gonzalez, F. (2007) “Effective Property Rights, Conflict and Growth,” Journal of Economic Theory 137, 127-39.

Gradstein, M. (2004) “Governance and Growth,” Journal of Development Economics 73, 505-18.

Greif, A. (2006) Institutions and the Path to the Modern Economy: Lessons from Medieval Trade, Cambridge University Press: Cambridge.

Hafer, C. (2006) "On the Origins of Property Rights: Conflict and Production in the State of Nature," Review of Economic Studies 73, 119-43.

Hirshleifer, J. (1991) “The Paradox of Power,” Economics and Politics 3, 177-200.

Hirshleifer, J. (1994) “The Dark Side of Force,” Economic Inquiry 32, 1-10.

Hirshleifer, J. (1995) “Anarchy and its Breakdown,” Journal of Political Economy 103, 26 -52.

Konrad, K. and D. Kovenock. (2009) "Multi-battle Contests," Games and Economic Behavior 66, 256-74.

Kumar, V. (2008) "Production, Appropriation and the Dynamic Emergence of Property Rights," mimeo, Indian Institute of Technology Kanpur.

Maxwell, J. and R. Reuveny. (2005) “Continuing Conflict," Journal of Economic Behavior and Organization 58, $30-52$.

McBride, M., G. Milante, and S. Skaperdas. (2011) "Peace and War with Endogenous State Capacity," Journal of Conflict Resolution 55, 446-68.

McBride, M. and S. Skaperdas. (2014) "Conflict, Settlement, and the Shadow of the Future," Journal of Economic Behavior and Organization 105, 75-89.

Muthoo, A. (2004) "A Model of the Origins of Basic Property Rights," Games and Economic Behavior 49, 288-312.

Neary, H. (1997) "Equilibrium Structure in an Economic Model of Conflict," Economic Inquiry $35,480-494$.

Skaperdas, S. (1992) "Cooperation, Conflict, and Power in the Absence of Property Rights," American Economic Review 82, 720-739.

Skaperdas, S. and C. Syropoulos. (1997) “The Distribution of Income in the Presence of Appropriative Activities," Economica 64,101-17.

Sonin, K. (2003) "Why the Rich may Favor Poor Protection of Property Rights," Journal of Comparative Economics 31, 715-31.

Tornell, A. (1997) "Economic Growth and Decline with Endogenous Property Rights," Journal of Economic Growth 2, 219-50.

Yoo, Y-H. (2013) "Stronger Property Rights Enforcement Does Not Hurt Social Welfare," Working paper 13-02, KDI School of Public Policy and Management, South Korea. 


\section{Supplementary Material for}

\section{"Appropriative Conflicts and the Evolution of Property Rights"}

\section{Proofs not for publication}

\section{ProOfS OF COROLLARY 3 AND PROPOSITION 4:}

We first determine how $W_{2}^{\dagger}(),.\left[W_{1}^{\dagger}()+.W_{2}^{\dagger}().\right], W_{1}^{\dagger}($.$) , and \left[W_{1}^{\dagger}()-.W_{2}^{\dagger}().\right]$ change in $\pi$. To simplify notation, we set $R=1$ as $R$ does not affect these comparative statics results.

We establish some preliminary facts. First, $W_{1}^{\dagger}($.$) and W_{2}^{\dagger}($.$) are continuous in all their arguments.$ Next, given any $\pi<1$, the Banditry equilibrium exists for all $\rho \leq \tilde{\rho}(\pi \mid \sigma)$ while the Hobbesian equilibrium exists for all $\rho>\tilde{\rho}(\pi \mid \sigma)$. Further, $\tilde{\rho}(\pi \mid \sigma)$ strictly decreases in $\pi$ and takes its maximum value of $0.5 \sigma /(1+\sigma)<0.25$ at $\pi=0$. Thus, for $\rho>0.5 \sigma /(1+\sigma)$, we obtain the Hobbesian equilibrium for all $\pi \in[0,1)$. Rearranging $\tilde{\rho}(\pi \mid \sigma) \equiv 0.5\{[(1-\pi) \sigma] /[1+\pi+(1-\pi) \sigma]\}$, we obtain the following result: for any $\rho \in(0,0.5 \sigma /(1+\sigma)]$, the Banditry equilibrium exists for all $\pi \in\left[0, \pi^{0}(\rho \mid \sigma)\right]$, while the Hobbesian equilibrium exists for all $\pi \in\left(\pi^{0}(\rho \mid \sigma), 1\right)$, where $\pi^{0}(\rho \mid \sigma) \equiv[\sigma-2 \rho(1+\sigma)] /[\sigma+$ $2 \rho(1-\sigma)] \in[0,1)$.

Consider the case where $\rho>0.5 \sigma /(1+\sigma)$ and thus the Hobbesian equilibrium obtains for all $\pi \in[0,1)$. In this case, $\partial W_{2}^{\dagger}(.) / \partial \pi=\sigma /[(1+\sigma)+(1-\sigma) \pi]^{2}-1 / 2+\rho$. As this expression is strictly falling in $\pi$, $W_{2}^{\dagger}$ (.) is strictly concave in $\pi$. So, there are the following three mutually exclusive cases to consider:

- $\quad \rho \in[1 / 2-\sigma / 4,1 / 2) \Leftrightarrow \partial W_{2}^{\dagger}(\sigma, \pi=1, R, \rho) / \partial \pi \geq 0$ : in this case $W_{2}^{\dagger}(\sigma, \pi, R, \rho)$ is uniquely maximized at $\pi=1$. [Note that there is no discontinuity in $W_{2}^{\dagger}(\sigma, \pi, R, \rho)$ at $\pi=1$.]

- $\rho \in\left(0.5 \sigma /(1+\sigma), 1 / 2-\sigma /(1+\sigma)^{2}\right] \Leftrightarrow \partial W_{2}^{\dagger}(\sigma, \pi=0, R, \rho) / \partial \pi \leq 0$ : in this case $W_{2}^{\dagger}(\sigma, \pi, R, \rho)$ is uniquely maximized at $\pi=0$.

- $\rho \in\left(1 / 2-\sigma /(1+\sigma)^{2}, 1 / 2-\sigma / 4\right) \Leftrightarrow \partial W_{2}^{\dagger}(\sigma, \pi=1, R, \rho) / \partial \pi<0<\partial W_{2}^{\dagger}(\sigma, \pi=0, R, \rho) / \partial \pi \Leftrightarrow$ : in this case $W_{2}^{\dagger}(\sigma, \pi, R, \rho)$ is uniquely maximized at $\pi=\widetilde{\pi}(\rho \mid \sigma)\left(\right.$ since $\partial W_{2}^{\dagger}(.) / \partial \pi=0$ at $\left.\pi=\widetilde{\pi}(\rho \mid \sigma)\right)$.

Next, we consider the case when $\rho \leq 0.5 \sigma /(1+\sigma)$. In this case, we obtain the Hobbesian equilibrium for all $\pi \in\left(\pi^{0}(\rho \mid \sigma), 1\right)$. In this sub-case, the Hobbesian equilibrium expression for $W_{2}^{\dagger}($.$) is strictly$ concave in $\pi$ and $\partial W_{2}^{\dagger}(\sigma, \pi=0, R, \rho) / \partial \pi<0$; thus $W_{2}^{\dagger}($.$) is strictly decreasing in \pi \in\left(\pi^{0}(\rho \mid \sigma), 1\right){ }^{73}$ We obtain the Banditry equilibrium for the case when $\left\{\rho \in(0,0.5 \sigma /(1+\sigma)]\right.$ and $\left.\pi \in\left[0, \pi^{0}(\rho \mid \sigma)\right]\right\}$. We next show that for these parameter values: $\partial W_{2}^{\dagger}(.) / \partial \pi<0$. Using the Banditry equilibrium

${ }^{73}$ Note that the Hobbesian equilibrium does not obtain here for $\pi \leq \pi^{0}(\rho \mid \sigma)$, and we only have: $W_{2}^{\dagger}(\sigma, \pi$, $R, \rho)=\left\{\pi \rho+\frac{[1-\pi][1+(1-\sigma) \pi]}{2[1+\sigma+(1-\sigma) \pi]}\right\} \cdot R$ for $\pi \in\left(\pi^{0}(\rho \mid \sigma), 1\right)$. But the function $\left\{\pi \rho+\frac{[1-\pi][1+(1-\sigma) \pi]}{2[1+\sigma+(1-\sigma) \pi]}\right\} \cdot R$ is welldefined for all $\pi \in[0,1]$. So, if we show that this function is strictly decreasing for $\pi \in[0,1]$, then we would have also shown that $W_{2}^{\dagger}(\sigma, \pi, R, \rho)$ is strictly decreasing for $\pi \in\left(\pi^{0}(\rho \mid \sigma), 1\right)$. 
expression for $W_{2}^{\dagger}($.$) , we get \partial W_{2}^{\dagger}(.) / \partial \pi=-(1-\sigma) / 2+\sigma \rho+\left\{[1+\pi+(1-\pi) \sigma]^{1 / 2} \cdot\left[(1-\sigma) \cdot[2(1-\pi) \sigma \rho]^{1 / 2}\right.\right.$ $\left.\left.-[\pi+(1-\pi) \sigma] \cdot(\sigma \rho) /[2(1-\pi) \sigma \rho]^{1 / 2}\right]-[\pi+(1-\pi) \sigma] \cdot[2(1-\pi) \sigma \rho]^{1 / 2}(1-\sigma) /\left[2(1+\pi+(1-\pi) \sigma)^{1 / 2}\right]\right\} /\{1+\pi$ $+(1-\pi) \sigma\}=-(1-\sigma) / 2+\sigma \rho+(\sigma \rho) .\{[\pi+(1-\pi) \sigma] \cdot[2(1-\sigma) \cdot(1-\pi)-1]+[3(1-\sigma) \cdot(1-\pi)-1]\} /\{[1+\pi+$ $\left.(1-\pi) \sigma]^{3 / 2} \cdot[2(1-\pi) \sigma \rho]^{1 / 2}\right\}$. Evaluating the previous expression at $\pi=0$, we get: $\partial W_{2}^{\dagger}(\pi=0) / \partial \pi=-$ $(1-\sigma) / 2+\sigma \rho-[2 \sigma \rho]^{1 / 2} \cdot\left[\sigma^{2}+\sigma-1\right] /[1+\sigma]^{3 / 2}$, which is easily shown to be strictly negative for all $\sigma$ $\in[0.5,1)$ and $\rho \in(0,0.5 \sigma /(1+\sigma)]$. Finally, straightforward differentiation followed by some algebraic re-arrangement helps us sign $\partial^{2} W_{2}^{\dagger}(.) / \partial \pi^{2}$ as negative, thus giving us the result that $\partial W_{2}^{\dagger}(.) / \partial \pi<0$ for the case when $\left\{\rho \in(0,0.5 \sigma /(1+\sigma)]\right.$ and $\left.\pi \in\left[0, \pi^{0}(\rho \mid \sigma)\right]\right\}$.

We thus conclude that when $\rho \in\left(0, \rho^{-}(\sigma)\right]$, where $\left.\rho^{-}(\sigma) \equiv 1 / 2-\sigma /(1+\sigma)^{2}\right), W_{2}^{\dagger}($.$) strictly decreases$ in $\pi$ and is maximized at $\pi=0$.

We now show that $\left[W_{1}^{\dagger}()+.W_{2}^{\dagger}().\right]$ strictly increases in $\pi \in[0,1)$. For the case when $\{\rho>\tilde{\rho}(\pi \mid \sigma)\}$ or $\left\{\rho \leq \tilde{\rho}(\pi \mid \sigma)\right.$ and $\left.\pi>\pi^{0}(\rho \mid \sigma)\right\}$ (i.e., when a Hobbesian equilibrium exists): $\left[W_{1}^{\dagger}()+.W_{2}^{\dagger}().\right]=$ $\{\pi+(1-\pi)[1+(1-\sigma) \pi] /[1+\sigma+(1-\sigma) \pi]\} \Rightarrow \partial\left(W_{1}^{\dagger}()+.W_{2}^{\dagger}().\right) / \partial \pi=2 \sigma /[1+\sigma+(1-\sigma) \pi]^{2}>0$. For the case when $\left\{\rho \leq \tilde{\rho}(\pi \mid \sigma)\right.$ and $\left.\pi \leq \pi^{0}(\rho \mid \sigma)\right\}$ (i.e., when a Banditry equilibrium exists): $\left[W_{1}^{\dagger}()+\right.$. $\left.W_{2}^{\dagger}().\right]=1-\sqrt{ }\{2(1-\pi) \sigma \rho /[1+\pi+(1-\pi) \sigma]\}$. Note that the denominator of the second term of the previous expression increases in $\pi$ while the numerator decreases in $\pi$, so the whole expression is easily seen to be increasing in $\pi$. So, in this case also we have that $\partial\left[W_{1}^{\dagger}()+.W_{2}^{\dagger}().\right] / \partial \pi>0$. Hence, $\left[W_{1}^{\dagger}()+.W_{2}^{\dagger}().\right]$ strictly increases in $\pi \in[0,1]$, and is maximized at $\pi=1$.

We next establish that $W_{1}^{\dagger}($.) strictly increases in $\pi$. It is readily established that for the case when $\{\rho>\tilde{\rho}(\pi \mid \sigma)\}$ or $\left\{\rho \leq \tilde{\rho}(\pi \mid \sigma)\right.$ and $\left.\pi>\pi^{0}(\rho \mid \sigma)\right\}: \partial W_{1}^{\dagger}(.) / \partial \pi=\sigma /[(1+\sigma)+(1-\sigma) \pi]^{2}-1 / 2+(1-\rho)$ $>0$. Further, since $W_{2}^{\dagger}\left(\right.$.) strictly decreases in $\pi$ while $\left[W_{1}^{\dagger}()+.W_{2}^{\dagger}().\right]$ strictly increases in $\pi$ for $\left\{\rho \leq \tilde{\rho}(\pi \mid \sigma)\right.$ and $\left.\pi \leq \pi^{0}(\rho \mid \sigma)\right\}$, it must be that in this case $W_{1}^{\dagger}($.$) strictly increases in \pi$.

We finally establish that $\left[W_{1}^{\dagger}()-.W_{2}^{\dagger}().\right]$ strictly increases in $\pi$. When $\{\rho>\tilde{\rho}(\pi \mid \sigma)\}$ or $\{\rho \leq \tilde{\rho}(\pi \mid$ $\sigma)$ and $\left.\pi>\pi^{0}(\rho \mid \sigma)\right\},\left[W_{1}^{\dagger}()-.W_{2}^{\dagger}().\right]=\pi .(1-2 \rho) \Rightarrow \partial\left(W_{1}^{\dagger}()-.W_{2}^{\dagger}().\right) / \partial \pi>0$. When $\{\rho \leq \tilde{\rho}(\pi \mid \sigma)$ and $\left.\pi \leq \pi^{0}(\rho \mid \sigma)\right\}, W_{2}^{\dagger}($.$) strictly decreases in \pi$ and $W_{1}^{\dagger}($.$) strictly increases in \pi$, implying that $\left[W_{1}^{\dagger}()-.W_{2}^{\dagger}().\right]$ strictly increases in $\pi$.

Thus, we have proved that $\partial W_{1}^{\dagger}(.) / \partial \pi>\max \left\{0, \partial W_{2}^{\dagger}(.) / \partial \pi\right\}$. Henceforth, we allow any $R>0$. Note that $\left[W_{2}^{\dagger}(.) / R_{2}\right]=\left[W_{1}^{\dagger}(.) / R_{1}\right]=1$ when $\pi=1$. Thus, when $\rho \in\left(0, \rho^{-}(\sigma)\right],\left[W_{2}^{\dagger}(.) / R_{2}\right]>1>$ $\left[W_{1}^{\dagger}\left(\right.\right.$.) $\left./ R_{1}\right]$ for all $\pi<1$ since in this case, $W_{2}^{\dagger}$ (.) increases as $\pi$ falls while $W_{1}^{\dagger}($.) decreases as $\pi$ falls. Next, we prove that $\left[W_{2}^{\dagger}(.) / R_{2}\right]>\left[W_{1}^{\dagger}(.) / R_{1}\right]$ for all $\pi<1$ and $\rho \in\left(\rho^{-}(\sigma), 1 / 2\right)$. In this case, where the Hobbesian equilibrium obtains for all $\pi<1$, let us suppose that $\left[W_{2}^{\dagger}(.) / R_{2}\right] \leq\left[W_{1}^{\dagger}(.) / R_{1}\right]$. Then, using the expressions for $W_{1}^{\dagger}($.$) and W_{2}^{\dagger}($.) in Proposition 2 and doing straightforward algebra gives us that $\rho \geq 1 / 2$, which is a contradiction to the fact that $\rho<1 / 2$. Hence, our supposition is incorrect. 
We thus have $\left[W_{2}^{\dagger}(.) / R_{2}\right]>\left[W_{1}^{\dagger}(.) / R_{1}\right]$ for all $\pi<1$ and $\rho \in(0,1 / 2)$.

To establish that $b_{12}{ }^{\dagger}(\sigma, \pi, R, \rho)>0$ for all $\pi<1$ and $\rho \in(0,1 / 2)$, we first establish that $\left[B_{2}^{\dagger}(.) / R_{2}\right]<$ $\left[B_{1}^{\dagger}(.) / R_{1}\right]$. This is true when the Banditry equilibrium obtains since then $B_{2}^{\dagger}()=$.0 . Note that in the Hobbesian equilibrium we have that: $G_{2}^{\dagger}()=.G_{1}^{\dagger}(.) \Rightarrow\left[G_{2}^{\dagger}(.) / R_{2}\right]>\left[G_{1}^{\dagger}(.) / R_{1}\right]$, hence: $\left[B_{2}^{\dagger}()\right.$. $\left./ R_{2}\right]=\left[\left(R_{2}-G_{2}^{\dagger}().\right) / R_{2}\right]<\left[B_{1}^{\dagger}(.) / R_{1}\right]=\left[\left(R_{1}-G_{1}^{\dagger}().\right) / R_{1}\right]$. Hence, we have that: $\left[B_{2}^{\dagger}(.) / R_{2}\right]<\left[B_{1}^{\dagger}()\right.$. $\left./ R_{1}\right]$ for all $\pi<1$ and $\rho \in(0,1 / 2)$. Now, by definition of $b_{12}^{\dagger}(\sigma, \pi, R, \rho)$, we have that: $W_{2}^{\dagger}()=.\left[B_{2}^{\dagger}()\right.$. $\left.+b_{12}{ }^{\dagger}(\sigma, \pi, R, \rho)\right]$ and $W_{1}^{\dagger}()=.\left[B_{1}^{\dagger}()-.b_{12}^{\dagger}(\sigma, \pi, R, \rho)\right]$. So, we have: $\left\{W_{2}^{\dagger}(.) / R_{2}\right\}>\left\{W_{1}^{\dagger}(.) / R_{1}\right\} \Rightarrow$ $\left\{\left[B_{2}^{\dagger}()+.b_{12}{ }^{\dagger}(\sigma, \pi, R, \rho)\right] / R_{2}\right\}>\left\{\left[B_{1}^{\dagger}()-.b_{12}^{\dagger}(\sigma, \pi, R, \rho)\right] / R_{1}\right\} \Rightarrow b_{12}^{\dagger}(\sigma, \pi, R, \rho) .\left[1 / R_{1}+1 / R_{2}\right]>$ $\left[\left\{B_{1}^{\dagger}(.) / R_{1}\right\}-\left\{B_{2}^{\dagger}(.) / R_{2}\right\}\right]>0$, hence we get that: $b_{12}^{\dagger}(\sigma, \pi, R, \rho)>0$.

Finally, consider the rights negotiation game given $\Pi=[0,1]$. Given the poorer community 2's optimal rights choice $\pi^{\dagger}(\rho \mid \sigma)$ for any $\rho \in(0,1 / 2)$ : if community 1 announces $s_{1} \leq \pi^{\dagger}(\rho \mid \sigma)$, then it is optimal for community 2 to continue by announcing any $s_{2} \in\left[s_{1}, 1\right]$ [since $W_{2}^{\dagger}($.) is single-peaked at $\left.\pi=\pi^{\dagger}(\rho \mid \sigma)\right]$ leading to the establishment of $s_{1}\left(\leq \pi^{\dagger}(\rho \mid \sigma)\right)$ as the rights regime; if community 1 announces $s_{1}>\pi^{\dagger}(\rho \mid \sigma)$, then it is optimal for community 2 to continue by announcing $s_{2}=\pi^{\dagger}(\rho \mid \sigma)$ leading to the establishment of $\pi^{\dagger}(\rho \mid \sigma)$ as the rights regime. Since $W_{1}^{\dagger}($.$) is strictly increasing in \pi$, in equilibrium, either community 1 will announce any $s_{1} \in\left(\pi^{\dagger}(\rho \mid \sigma), 1\right]$ and community 2 will optimally continue by announcing $s_{2}=\pi^{\dagger}(\rho \mid \sigma)$, or community 1 will announce $s_{1}=\pi^{\dagger}(\rho \mid \sigma)$ and community 2 will optimally continue by announcing any $s_{2} \in\left[\pi^{\dagger}(\rho \mid \sigma), 1\right]$, each case leading to the unique $S P N E$ outcome of the establishment of $\pi^{\dagger}(\rho \mid \sigma)$ as the rights regime.

\section{ProOF OF Proposition 5:}

The detailed proof of the proposition is available in Goel (2017). ${ }^{74}$ Here we simply record that in the guns-and-butter game with no commitment, the conclusions of Lemma 1 hold, and given $\pi \in[0$, 1), the unique equilibrium payoffs of the two communities in the guns-and-butter game are:

$$
\begin{aligned}
& W_{1}{ }^{[N]}(\pi, \rho)= \begin{cases}\{\pi(1-\rho)+[(1-\pi) \sigma] / 4\}\left[R_{1}+R_{2}\right] & \text { for } \rho \in\left(\rho^{N}(\pi, \sigma), 1 / 2\right], \\
\{\sqrt{ }[\pi+(1-\pi) \sigma]-\sqrt{ }[(1-\pi) \sigma \rho]\}^{2}\left[R_{1}+R_{2}\right] & \text { for } \rho \in\left(0, \rho^{N}(\pi, \sigma)\right],\end{cases} \\
& W_{2}{ }^{[N]}(\pi, \rho)= \begin{cases}\{\pi \rho+[(1-\pi) \sigma] / 4\}\left[R_{1}+R_{2}\right] & \text { for } \rho \in\left(\rho^{N}(\pi, \sigma), 1 / 2\right], \\
\{\sqrt{[}[\pi+(1-\pi) \sigma][(1-\pi) \sigma \rho]]-(1-\pi) \sigma \rho\}\left[R_{1}+R_{2}\right] & \text { for } \rho \in\left(0, \rho^{N}(\pi, \sigma)\right] ;\end{cases}
\end{aligned}
$$

where $\rho^{N}(\pi, \sigma) \equiv 0.25\{[(1-\pi) \sigma] /[\pi+(1-\pi) \sigma]\} \in(0,0.25] \forall \pi \in[0,1)$. If $\pi=1$, then $W_{i}^{[N]}(\pi, \rho)=R_{i}$. Then, differentiation of the payoff functions with respect to $\pi$ (for $\rho$ in $\left(\rho^{N}(\pi, \sigma), 1 / 2\right]$ and in $(0$, $\left.\left.\rho^{N}(\pi, \sigma)\right]\right)$ proves that $W_{1}{ }^{[N]}(\pi, \rho)$ is maximized at 1 and $W_{2}{ }^{[N]}(\pi, \rho)$ is maximized at $\pi^{[N]}(\rho \mid \sigma)$.

${ }^{74}$ Goel, B. (2017) “Contest Theory and its Applications to Development and Political Economy," under preparation, Indian Institute of Management Calcutta. 


\section{PROOF OF PROPOSITION 6:}

Let communities $i, j=1,2$ with $i \neq j$. Also, define $R \equiv\left(R_{1}+R_{2}\right)$. We restrict attention to $\rho>0.25$, hence: $0.75 R>R_{1}>R_{2}>0.25 R$. It is easy to see that (the counterpart of) Lemma 1 and its proof go though without change with this alternative specification: Given $\pi \in[0,1)$ and given a guns-andbutter output vector $\left\{\left(g_{1}, B_{1}\right),\left(g_{2}, B_{2}\right)\right\}$ such that $\left[B_{1}+B_{2}\right]>0$ and $\left[g_{1} \times g_{2}\right]>0$, community $i$ 's final payoff (after the exchange of the butter bribe) is:

$$
\left\{\left[0.5(1-\pi)(1-\sigma)\left(B_{1}+B_{2}\right)\right]+\left[\pi B_{i}+(1-\pi)\left[g_{i} /\left(g_{1}+g_{2}\right)\right] . \sigma \cdot\left(B_{1}+B_{2}\right)\right]\right\} .^{75}
$$

The proof of (the counterpart of) Proposition 2 requires minor adjustments, which are described next. If $\pi=1$, then in the unique guns-and-butter equilibrium: $W_{k}^{\dagger}=B_{k}^{\dagger}=R_{k}$ for $k=1,2$. Given $\pi$ $\in[0,1)$ we want to determine all the guns-and-butter equilibria. It is straightforward to see that setting $\left[G_{i}+B_{i}\right]<R_{i}$ is a strictly dominated strategy. Henceforth we will take $\left[G_{i}+B_{i}\right]=R_{i}$ and consider $G_{i}$ as community $i$ 's sole choice variable; further, we will denote an equilibrium simply by $\left\{G_{1}^{\dagger}, G_{2}^{\dagger}\right\}$. We will first establish that there exists a unique candidate 'interior equilibrium' $\left\{G_{1}^{\dagger}\right.$, $\left.G_{2}^{\dagger}\right\} \in\left(0, R_{1}\right) \times\left(0, R_{2}\right)$. Given a conjecture about rival $G_{j} \in\left(0, R_{j}\right)$ (resp., $\left.G_{j}=R_{j}\right)$, community $i$ must be maximizing over all $G_{i} \in\left(0, R_{i}\right]$ (resp., $\left.G_{i} \in\left[0, R_{i}\right]\right)$, its payoff $V_{i}\left(G_{i}, G_{j}\right) \equiv\{[0.5(1-\pi)(1-$ $\left.\left.\sigma)\left(B_{1}+B_{2}\right)\right]+\left[\pi \cdot B_{i}+(1-\pi)\left[G_{i}^{\gamma} /\left(G_{1}^{\gamma}+G_{2}^{\gamma}\right)\right] \cdot \sigma \cdot\left(B_{1}+B_{2}\right)\right]\right\}$, where $\left[G_{k}+B_{k}\right]=R_{k}$ for $k=1,2$. Now, any 'interior equilibrium' $\left\{G_{1}^{\dagger}, G_{2}^{\dagger}\right\} \in\left(0, R_{1}\right) \times\left(0, R_{2}\right)$ must simultaneously solve: $\partial V_{1}\left(G_{1}^{\dagger}, G_{2}^{\dagger}\right) / \partial G_{1}=$ 0 and $\partial V_{2}\left(G_{2}^{\dagger}, G_{1}^{\dagger}\right) / \partial G_{2}=0$. Solving this system of equations, we obtain the unique candidate 'interior equilibrium': $G_{1}^{\dagger}(\sigma, \pi, R, \rho \mid \gamma)=G_{2}^{\dagger}(\sigma, \pi, R, \rho \mid \gamma)=\tilde{\rho}(\pi \mid \sigma \gamma) \cdot R$, where: $\tilde{\rho}(\pi \mid \sigma \gamma) \equiv$ $0.5\{[(1-\pi) \sigma \gamma] /[1+\pi+(1-\pi) \sigma \gamma]\} \quad(<0.25<\rho$, since $\sigma \gamma<1)$.

Is this candidate equilibrium actually an equilibrium? Keeping $j$ 's choice fixed at $G_{j}^{\dagger}=\tilde{\rho}(\pi \mid \sigma \gamma) \cdot R$, we want to determine whether community $i$ can profitably deviate from $G_{i}^{\dagger}=\tilde{\rho}(\pi \mid \sigma \gamma) \cdot R$. It can be shown that $\operatorname{sign}\left\{\partial^{2} V_{i}\left(G_{i}, G_{j}\right) / \partial\left(G_{i}\right)^{2}\right\}=\operatorname{sign}\left\{\left[B_{1}+B_{2}\right] \times\left[\left((\gamma-1) / G_{i}\right)-\left(2 \gamma G_{i}^{(\gamma-1)} /\left(G_{1}^{\gamma}+G_{2}^{\gamma}\right)\right)\right]-2\right\}$. For $\gamma$ $\leq 1$, the expression in the second curly brackets is strictly negative and so $V_{i}\left(G_{i}, G_{j}^{\dagger}\right)$ is strictly concave in $G_{i}$ and hence the candidate equilibrium is an equilibrium. Next, we consider the case when $\gamma \in(1,1 / \sigma)$. Given $\left[B_{1}+B_{2}\right]>0$, it is straightforward to check that the second expression in curly brackets is strictly positive when $G_{i}$ is close to 0 and it can change sign a maximum of one time as $G_{i}$ is increased from 0 up to $R_{i}$, keeping $G_{j}>0$ fixed. So, keeping $G_{j}>0$ fixed, either $V_{i}\left(G_{i}\right.$, $\left.G_{j}\right)$ is convex for all $G_{i}$ or it goes from being convex when $G_{i}$ is close to 0 to being concave at larger values of $G_{i}$. Further, it can be verified that $\operatorname{sign}\left\{\partial^{2} V_{i}\left(G_{i}, G_{j}=G_{i}\right) / \partial\left(G_{i}\right)^{2}\right\}=(-)$. [So, $G_{i}=\tilde{\rho}(\pi \mid$ $\sigma \gamma) \cdot R$ is a local maxima for $V_{i}\left(G_{i}, \tilde{\rho}(\pi \mid \sigma \gamma) . R\right)$.] Hence, the only deviation by community $i$ from the candidate equilibrium $\left\{G_{1}^{\dagger}, G_{2}^{\dagger}\right\}=\{[\tilde{\rho}(\pi \mid \sigma \gamma) \cdot R],[\tilde{\rho}(\pi \mid \sigma \gamma) \cdot R]\}$ that we need to check is the deviation of $G_{i}$ to an 'arbitrarily small but positive' $v$ (since $i$ 's payoff $V_{i}\left(G_{i}, G_{j}^{\dagger}\right)$ falls

${ }^{75}$ It is easy to verify that this expression also provides community $i$ 's final payoff when $B_{j}=0$ and $g_{i}=0$. 
discontinuously at $G_{i}=0$ (since: $B_{j}^{\dagger}>0$ ), a deviation to $G_{i}=0$ will be strictly dominated by a deviation to $G_{i}=v$ ). Given $\gamma<2$ (since we have: $\gamma<1 / \sigma$ and $\sigma \in[1 / 2,1)$ ), it is easy to verify that $V_{i}(\tilde{\rho}(\pi \mid \sigma \gamma) \cdot R, \tilde{\rho}(\pi \mid \sigma \gamma) \cdot R)>V_{i}(\mathrm{v}, \tilde{\rho}(\pi \mid \sigma \gamma) \cdot R)$, and so no profitable deviation from the candidate equilibrium exists. Hence, $G_{1}^{\dagger}(\sigma, \pi, R, \rho \mid \gamma)=G_{2}^{\dagger}(\sigma, \pi, R, \rho \mid \gamma)=\tilde{\rho}(\pi \mid \sigma \gamma) \cdot R$ is indeed an equilibrium. But is it the unique equilibrium?

We next establish that there cannot exist any corner equilibrium. The only corner equilibria that could possibly exist are those where: $G_{i}^{\dagger}=R_{i}$ and $G_{j}^{\dagger} \in\left(0, R_{j}\right)$. Given $\rho>0.25$ and $\gamma<1 / \sigma$, it can be shown through fairly straightforward algebra that when community $j$ optimally responds to its conjectured $G_{i}=R_{i}$, then taking that response as community $i$ 's conjecture $i$ 's best response is not $G_{i}$ $=R_{i}$. Hence, there exists no corner equilibrium.

So, the unique SPNE payoffs for communities 1 and 2 are determined by setting $W_{i}^{\dagger}(\sigma, \pi, R, \rho \mid \gamma)=$ $V_{i}(\tilde{\rho}(\pi \mid \sigma \gamma) \cdot R, \tilde{\rho}(\pi \mid \sigma \gamma) \cdot R)$, and they are respectively given, for all $\pi \in[0,1)$, as:

$W_{1}^{\dagger}(\sigma, \pi, R, \rho \mid \gamma)=\left\{\pi(1-\rho)+\frac{[1-\pi][1+(1-\sigma \gamma) \pi]}{2[1+\sigma \gamma+(1-\sigma \gamma) \pi]}\right\} \cdot R$,

$W_{2}^{\dagger}(\sigma, \pi, R, \rho \mid \gamma)=\left\{\pi \rho+\frac{[1-\pi][1+(1-\sigma \gamma) \pi]}{2[1+\sigma \gamma+(1-\sigma \gamma) \pi]}\right\} \cdot R$.

Notice that the above expressions are essentially the same as those given in Proposition 2 (for the case when $\rho$ is "large") with $\sigma<1$ simply being replaced by $[\sigma \gamma]<1$. Hence, the comparative statics for $W_{i}^{\dagger}(\pi \mid \gamma)$ w.r.t. $\pi$ are similarly established, and give us that:

Community 1 will strictly prefer perfect property rights $\forall \rho>0.25$; while community 2 will strictly prefer anarchy $\forall \rho \in\left(0.25, \rho^{-}(\sigma \gamma)\right]$, strictly prefer $\widetilde{\pi}(\rho \mid \sigma \gamma) \in(0,1) \forall \rho \in\left(\rho^{-}(\sigma \gamma), \rho^{+}(\sigma \gamma)\right)$, and strictly prefer perfect property rights $\forall \rho \in\left[\rho^{+}(\sigma \gamma), 0.5\right)$.

\section{PROOF OF LEMMA 7:}

Given feasible structural parameters including a well behaved $R L M$, let the inheritance vector in generation $t$ be $\left\{R_{1}^{*}(t), R_{2}^{*}(t)\right\}$ with $R^{*}(t) \equiv\left[R_{1} *(t)+R_{2}^{*}(t)\right]>0$ and $\rho^{*}(t) \equiv R_{2}^{*}(t) / R^{*}(t) \in(0,1 / 2)$. The two communities in generation $t$ aim to myopically maximize their own current consumptions. For any choice of $\pi(t)$, the immediate implication of Proposition 2 is that $B_{1} *(t)>B_{2} *(t)$ and $G_{1} *(t)$ $\geq G_{2}^{*}(t)$. Then the fact that $W_{1}^{*}(t) \geq W_{2}^{*}(t)$ follows since each community $i$ 's final consumption $W_{i}^{*}(t)$ is $\{$ half of net bargaining surplus + own outside option $\} \equiv\left\{\left[0.5(1-\pi(t))(1-\sigma)\left(B_{1}^{*}(t)+\right.\right.\right.$ $\left.\left.\left.B_{2} *(t)\right)\right]+\left[\pi(t) \cdot B_{i}^{*}(t)+(1-\pi(t))\left[G_{i}^{*}(t) /\left(G_{1} *(t)+G_{2} *(t)\right)\right] \sigma\left(B_{1} *(t)+B_{2} *(t)\right)\right]\right\}$. Then, as the proof of Proposition 4 clarifies, the equilibrium consumption of each community will be single-peaked in property rights, and so, for each community there will be a unique optimal level of property rights in the inherited rights space. In the rights negotiation game, community 2 will be able to establish its uniquely optimal choice. In this way, the generation $t$ outcome vector will be uniquely determined as a function of generation $t$ 's inheritance vector. The unique generation $t$ outcome vector will then 
determine the unique inheritance vector of generation $t+1$, in which the rights negotiation space will be a compact subset of $[0,1]$ by construction, and (given the well-behaved $R L M$ ) it will be the case that $R_{1} *(t+1)>R_{2} *(t+1)$ since $R_{1} *(t)>R_{2} *(t), B_{1}^{*}(t)>B_{2} *(t)$ and $W_{1}^{*}(t) \geq W_{2}^{*}(t)$.

\section{PRoOf OF FoOTnOTE 53:}

For the first two RLM structures, i.e., the non-linear and non-separable ones, we will be considering the equilibrium induced in the guns-and-butter game in any single generation given resource endowments $R_{1}>R_{2}>0$ (or equivalently, given: $R \equiv\left[R_{1}+R_{2}\right]>0$ and $\rho \equiv R_{2} / R \in(0,0.5)$ ) and established property rights $\pi$ in the generation. To conveniently denote the guns-and-butter equilibrium quantities in the generation, we will use the simpler notation: $\left(B_{i}, W_{i}\right)$ for $i=1,2$ (specifically, while representing the equilibrium quantities, we will suppress the use of "daggers" and the dependence on various arguments). Using the resource endowments and the guns-andbutter equilibrium quantities in the current generation, we aim to determine the properties of resource inequality bequeathed to the next generation in the continuation equilibrium of the game.

First, consider the following non-linear generalization of the linear homogeneous $R L M: F\left(R_{i}, B_{i}\right.$, $\left.W_{i}\right)=\left[x .\left(R_{i}\right)^{\chi}+y \cdot\left(B_{i}\right)^{\chi}+z \cdot\left(W_{i}\right)^{\chi}\right]$ with $\chi>0, x \geq 1, y>0, z>0$.

Suppose it is the case that $\pi<1$ and $\rho \in(\tilde{\rho}(\pi \mid \sigma), 0.5)$. The numerator of the derivative of $\left[y \cdot\left(B_{2}\right)^{\chi}\right.$ $\left.+z \cdot\left(W_{2}\right)^{\chi}\right] /\left[y \cdot\left(B_{1}\right)^{\chi}+z \cdot\left(W_{1}\right)^{\chi}\right]$ w.r.t. $\chi$ is (with the denominator of the derivative simply being $\left.\left[y \cdot\left(B_{1}\right)^{\chi}+z \cdot\left(W_{1}\right)^{\chi}\right]^{2}>0\right):\left[y \cdot\left(B_{1}\right)^{\chi}+z \cdot\left(W_{1}\right)^{\chi}\right] \cdot\left[y \cdot \ln \left(B_{2}\right) \cdot\left(B_{2}\right)^{\chi}+z \cdot \ln \left(W_{2}\right) \cdot\left(W_{2}\right)^{\chi}\right]-\left[y \cdot\left(B_{2}\right)^{\chi}+\right.$ $\left.z \cdot\left(W_{2}\right)^{\chi}\right] \cdot\left[y \cdot \ln \left(B_{1}\right) \cdot\left(B_{1}\right)^{\chi}+z \cdot \ln \left(W_{1}\right) \cdot\left(W_{1}\right)^{\chi}\right]=y^{2} \cdot\left(B_{1}\right)^{\chi} \cdot\left(B_{2}\right)^{\chi} \cdot \ln \left(B_{2} / B_{1}\right)+y \cdot z \cdot\left(B_{1}\right)^{\chi} \cdot\left(W_{2}\right)^{\chi} \cdot \ln \left(W_{2} / B_{1}\right)+$ $y \cdot z \cdot\left(B_{2}\right)^{\chi} \cdot\left(W_{1}\right)^{\chi} \cdot \ln \left(B_{2} / W_{1}\right)+z^{2} \cdot\left(W_{1}\right)^{\chi} \cdot\left(W_{2}\right)^{\chi} \cdot \ln \left(W_{2} / W_{1}\right)$. It is easy to verify the following equilibrium relationships: $B_{2}<W_{2} \leq W_{1}<B_{1}$, and so: $\left(B_{2} / B_{1}\right)<1,\left(W_{2} / B_{1}\right)<1,\left(B_{2} / W_{1}\right)<1$ and $\left(W_{2} / W_{1}\right) \leq 1$. Hence, the derivative of $\left[y \cdot\left(B_{2}\right)^{\chi}+z \cdot\left(W_{2}\right)^{\chi}\right] /\left[y \cdot\left(B_{1}\right)^{\chi}+z \cdot\left(W_{1}\right)^{\chi}\right]$ w.r.t. $\chi$ is negative and so $\left[y \cdot\left(B_{2}\right)^{\chi}+\right.$ $\left.z .\left(W_{2}\right)^{\chi}\right] /\left[y \cdot\left(B_{1}\right)^{\chi}+z \cdot\left(W_{1}\right)^{\chi}\right]$ is decreasing in $\chi$. First, consider the case where $\{y / z>(2-\sigma) / \sigma\}$ : With the linear homogeneous $R L M$, i.e., with $\chi=1$, when $\{y / z>(2-\sigma) / \sigma\}$, and given $\pi<1$ and $\rho$ $\in(\tilde{\rho}(\pi \mid \sigma), 0.5)$, then we had: $\left[y \cdot\left(B_{2}\right)^{\chi}+z \cdot\left(W_{2}\right)^{\chi}\right] /\left[y \cdot\left(B_{1}\right)^{\chi}+z \cdot\left(W_{1}\right)^{\chi}\right]<R_{2} / R_{1}$, hence this is also true for $\chi>1$ (since $\left[y \cdot\left(B_{2}\right)^{\chi}+z \cdot\left(W_{2}\right)^{\chi}\right] /\left[y \cdot\left(B_{1}\right)^{\chi}+z \cdot\left(W_{1}\right)^{\chi}\right]$ is decreasing in $\left.\chi\right)$. Further, $\left[x \cdot\left(R_{2}\right)^{\chi}\right] /\left[x .\left(R_{1}\right)^{\chi}\right]<R_{2} / R_{1}$ when $\chi>1$. So, we have that: $\left[x \cdot\left(R_{2}\right)^{\chi}+y \cdot\left(B_{2}\right)^{\chi}+z \cdot\left(W_{2}\right)^{\chi}\right] /\left[x \cdot\left(R_{1}\right)^{\chi}+\right.$ $\left.y \cdot\left(B_{1}\right)^{\chi}+z \cdot\left(W_{1}\right)^{\chi}\right]<R_{2} / R_{1}$ when $\chi>1$. Second, consider the case where $\{y / z<1 / \sigma\}$ : With the linear homogeneous $R L M$, i.e., with $\chi=1$, when $\{y / z<1 / \sigma\}$, and given $\pi<1$ and $\rho \in(\tilde{\rho}(\pi \mid \sigma)$, 0.5), then we had: $\left[y \cdot\left(B_{2}\right)^{\chi}+z \cdot\left(W_{2}\right)^{\chi}\right] /\left[y \cdot\left(B_{1}\right)^{\chi}+z \cdot\left(W_{1}\right)^{\chi}\right]>R_{2} / R_{1}$, hence this is also true for $\chi<1$ (since $\left[y \cdot\left(B_{2}\right)^{\chi}+z \cdot\left(W_{2}\right)^{\chi}\right] /\left[y \cdot\left(B_{1}\right)^{\chi}+z \cdot\left(W_{1}\right)^{\chi}\right]$ is decreasing in $\left.\chi\right)$. Further, $\left[x \cdot\left(R_{2}\right)^{\chi}\right] /\left[x \cdot\left(R_{1}\right)^{\chi}\right]>R_{2} / R_{1}$ when $\chi<1$. So, we have that: $\left[x \cdot\left(R_{2}\right)^{\chi}+y \cdot\left(B_{2}\right)^{\chi}+z \cdot\left(W_{2}\right)^{\chi}\right] /\left[x \cdot\left(R_{1}\right)^{\chi}+y \cdot\left(B_{1}\right)^{\chi}+z \cdot\left(W_{1}\right)^{\chi}\right]>R_{2} / R_{1}$ when $\chi<1$ (This is similarly seen to be true for the specific case when: $\pi<1$ and $\rho=\tilde{\rho}(\pi \mid \sigma)$ ). Next, suppose it is the case that $\pi<1$ and $\rho \leq \tilde{\rho}(\pi \mid \sigma)$. The numerator of the derivative of $\left[x .\left(R_{2}\right)^{\chi}\right.$ 
$\left.+y \cdot\left(B_{2}\right)^{\chi}+z \cdot\left(W_{2}\right)^{\chi}\right] /\left[x \cdot\left(R_{1}\right)^{\chi}+y \cdot\left(B_{1}\right)^{\chi}+z \cdot\left(W_{1}\right)^{\chi}\right]$ w.r.t. $\chi$ is (with the denominator of the derivative simply being $\left.\left[x \cdot\left(R_{1}\right)^{\chi}+y \cdot\left(B_{1}\right)^{\chi}+z \cdot\left(W_{1}\right)^{\chi}\right]^{2}>0\right):\left[x \cdot\left(R_{1}\right)^{\chi}+y \cdot\left(B_{1}\right)^{\chi}+z \cdot\left(W_{1}\right)^{\chi}\right] \cdot\left[x \cdot \ln \left(R_{2}\right) \cdot\left(R_{2}\right)^{\chi}+\right.$ $\left.y \cdot \ln \left(B_{2}\right) \cdot\left(B_{2}\right)^{\chi}+z \cdot \ln \left(W_{2}\right) \cdot\left(W_{2}\right)^{\chi}\right]-\left[x \cdot\left(R_{2}\right)^{\chi}+y \cdot\left(B_{2}\right)^{\chi}+z \cdot\left(W_{2}\right)^{\chi}\right] \cdot\left[x \cdot \ln \left(R_{1}\right) \cdot\left(R_{1}\right)^{\chi}+y \cdot \ln \left(B_{1}\right) \cdot\left(B_{1}\right)^{\chi}+\right.$ $\left.z \cdot \ln \left(W_{1}\right) \cdot\left(W_{1}\right)^{\chi}\right]=x^{2} \cdot\left(R_{1}\right)^{\chi} \cdot\left(R_{2}\right)^{\chi} \cdot \ln \left(R_{2} / R_{1}\right)+x \cdot y \cdot\left(R_{1}\right)^{\chi} \cdot\left(B_{2}\right)^{\chi} \cdot \ln \left(B_{2} / R_{1}\right)+x \cdot z \cdot\left(R_{1}\right)^{\chi} \cdot\left(W_{2}\right)^{\chi} \cdot \ln \left(W_{2} / R_{1}\right)+(6$ more similar terms). It is easy to verify the following equilibrium relationships: $0=B_{2}<R_{2}<W_{2}$ $\leq W_{1}<B_{1}<R_{1}$, and so: $\left(R_{2} / R_{1}\right)<1,\left(B_{2} / R_{1}\right)<1,\left(W_{2} / R_{1}\right)<1,\left(R_{2} / B_{1}\right)<1,\left(B_{2} / B_{1}\right)<1,\left(W_{2} / B_{1}\right)<1$, $\left(R_{2} / W_{1}\right)<1,\left(B_{2} / W_{1}\right)<1$ and $\left(W_{2} / W_{1}\right) \leq 1$. Hence, the derivative of $\left[x .\left(R_{2}\right)^{\chi}+y \cdot\left(B_{2}\right)^{\chi}+z .\left(W_{2}\right)^{\chi}\right] /$ $\left[x .\left(R_{1}\right)^{\chi}+y \cdot\left(B_{1}\right)^{\chi}+z \cdot\left(W_{1}\right)^{\chi}\right]$ w.r.t. $\chi$ is negative and so $\left[x \cdot\left(R_{2}\right)^{\chi}+y \cdot\left(B_{2}\right)^{\chi}+z \cdot\left(W_{2}\right)^{\chi}\right] /\left[x \cdot\left(R_{1}\right)^{\chi}+y \cdot\left(B_{1}\right)^{\chi}\right.$ $\left.+z .\left(W_{1}\right)^{\chi}\right]$ is decreasing in $\chi$. First, consider the case where $\{y / z>(2-\sigma) / \sigma\}$ : With the linear homogeneous $R L M$, i.e., with $\chi=1$, when $\{y / z>(2-\sigma) / \sigma\}$, and given $\pi<1$ and $\rho \leq \tilde{\rho}(\pi \mid \sigma)$, then we had: $\left[x \cdot\left(R_{2}\right)^{\chi}+y \cdot\left(B_{2}\right)^{\chi}+z \cdot\left(W_{2}\right)^{\chi}\right] /\left[x \cdot\left(R_{1}\right)^{\chi}+y \cdot\left(B_{1}\right)^{\chi}+z \cdot\left(W_{1}\right)^{\chi}\right]<\tilde{\rho}(\pi \mid \sigma) /(1-\tilde{\rho}(\pi \mid \sigma))$, hence this is also true for $\chi>1$ (since $\left[x \cdot\left(R_{2}\right)^{\chi}+y \cdot\left(B_{2}\right)^{\chi}+z \cdot\left(W_{2}\right)^{\chi}\right] /\left[x \cdot\left(R_{1}\right)^{\chi}+y \cdot\left(B_{1}\right)^{\chi}+z \cdot\left(W_{1}\right)^{\chi}\right]$ is decreasing in $\chi)$. Second, consider the case where $\{y / z<1 / \sigma\}$ : With the linear homogeneous $R L M$, i.e., with $\chi=$ 1 , when $\{y / z<1 / \sigma\}$, and given $\pi=0$ and $\rho \leq \tilde{\rho}(\pi=0 \mid \sigma)$, then we had: $\left[x \cdot\left(R_{2}\right)^{\chi}+y \cdot\left(B_{2}\right)^{\chi}+z \cdot\left(W_{2}\right)^{\chi}\right]$ $/\left[x \cdot\left(R_{1}\right)^{\chi}+y \cdot\left(B_{1}\right)^{\chi}+z \cdot\left(W_{1}\right)^{\chi}\right]>R_{2} / R_{1}$, hence this is also true for $\chi<1$ (since $\left[x \cdot\left(R_{2}\right)^{\chi}+y \cdot\left(B_{2}\right)^{\chi}+\right.$ $\left.z \cdot\left(W_{2}\right)^{\chi}\right] /\left[x \cdot\left(R_{1}\right)^{\chi}+y \cdot\left(B_{1}\right)^{\chi}+z \cdot\left(W_{1}\right)^{\chi}\right]$ is decreasing in $\left.\chi\right)$.

Next, suppose it is the case that $\pi=1$ and $\rho \geq \rho^{+}(\sigma)$. We get: $\left[x \cdot\left(R_{2}\right)^{\chi}+y \cdot\left(B_{2}\right)^{\chi}+z \cdot\left(W_{2}\right)^{\chi}\right] /\left[x \cdot\left(R_{1}\right)^{\chi}\right.$ $\left.+y \cdot\left(B_{1}\right)^{\chi}+z \cdot\left(W_{1}\right)^{\chi}\right]=\left[(x+y+z) \cdot\left(R_{2}\right)^{\chi}\right] /\left[(x+y+z) \cdot\left(R_{1}\right)^{\chi}\right]=\left(R_{2}\right)^{\chi} /\left(R_{1}\right)^{\chi}$. For $\chi>1,\left(R_{2}\right)^{\chi} /\left(R_{1}\right)^{\chi}<$ $\left(R_{2}\right) /\left(R_{1}\right)$ and for $\chi<1,\left(R_{2}\right)^{\chi} /\left(R_{1}\right)^{\chi}>\left(R_{2}\right) /\left(R_{1}\right)$.

Hence, when $\{y / z>(2-\sigma) / \sigma\}$, then for $\chi>1$ the $R L M$ satisfies Property F-1.2, and so using Proposition 10 we have that the equilibrium path $\left\{\pi^{*}(t), \rho^{*}(t)\right\}_{t=1}^{\infty}$ devolves to anarchy for all $\rho(1)$ $\in(0,0.5)$. Alternatively, in the case where $\{y / z<1 / \sigma\}$, for $\chi<1$ the $R L M$ satisfies Property F-2, and so using Proposition 10 we have that the equilibrium path $\left\{\pi^{*}(t), \rho^{*}(t)\right\}_{t=1}^{\infty}$ attains perfect property rights for all $\rho(1) \in(0,0.5)$.

Second, consider the non-separable RLMs. Consider: $F\left(R_{i}, B_{i}, W_{i}\right)=\left[x \cdot R_{i}\left(\chi_{1}+\chi_{2}\right)+y \cdot R_{i}{ }^{\chi_{1}} \cdot B_{i}{ }^{\chi_{2}}\right]$ with $\left\{x \geq 1, y>0, \chi_{1}>0, \chi_{2}>0\right\}$ and $\left[\chi_{1}+\chi_{2}\right]>1$. We have for all $\pi \in[0,1]$ and $\rho \in(0,0.5)$ that: $B_{2} / B_{1} \leq R_{2} / R_{1}$, and $R_{2}{ }^{\left(\chi_{1}+\chi_{2}\right) / R_{1}}{ }^{\left(\chi_{1}+\chi_{2}\right)}<R_{2} / R_{1}$ since $\left[\chi_{1}+\chi_{2}\right]>1$, so $\left[x \cdot R_{2}{ }^{\left(\chi_{1}+\chi_{2}\right)+}\right.$ $\left.y \cdot R_{2}{ }^{\chi_{1}} \cdot B_{2}{ }^{\chi_{2}}\right] /\left[x \cdot R_{1}{ }^{\left(\chi_{1}+\chi_{2}\right)}+y \cdot R_{1}{ }^{\chi_{1}} \cdot B_{1}{ }^{\chi_{2}}\right]<R_{2} / R_{1}$. Hence, the homogeneous $R L M$ satisfies Property F-1.2, and so the equilibrium path $\left\{\pi^{*}(t), \rho^{*}(t)\right\}_{t=1}^{\infty}$ devolves to anarchy for all $\rho(1) \in(0$, 0.5). Next, consider: $F\left(R_{i}, B_{i}, W_{i}\right)=\left[x \cdot R_{i}{ }^{\left(\chi_{1}+\chi_{3}\right)}+z \cdot R_{i}{ }^{\chi_{1}} \cdot W_{i}{ }^{\chi_{3}}\right]$ with $\left\{x \geq 1, z>0, \chi_{1}>0, \chi_{3}>0\right\}$ and $\left[\chi_{1}+\chi_{3}\right] \leq 1$. We have for all $\pi \in[0,1]$ and $\rho \in(0,0.5)$ that: $W_{2} / W_{1} \geq R_{2} / R_{1}$, and

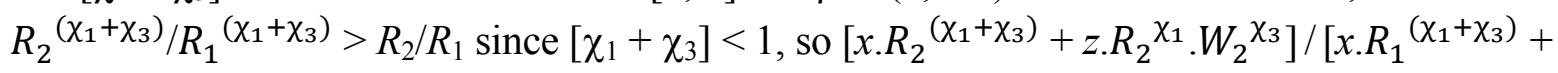
$\left.z . R_{1}{ }^{\chi_{1}} \cdot W_{1}{ }^{\chi_{3}}\right]>R_{2} / R_{1}$. Hence, the homogeneous $R L M$ satisfies Property F-2, and so the equilibrium path $\left\{\pi^{*}(t), \rho^{*}(t)\right\}_{t=1}^{\infty}$ attains perfect property rights for all $\rho(1) \in(0,0.5)$.

Third, consider the following time-varying linear $R L M: F\left(R_{i}, B_{i}, W_{i} ;(\rho, \pi)\right)=x(\rho, \pi) \cdot R_{i}+y(\rho, \pi) \cdot B_{i}$ 
$+z(\rho, \pi) . W_{i}$, where $x() \geq 1,. y()>$.0 and $z()>$.0 are continuous functions of their arguments (and where $\rho \in(0,0.5]$ and $\pi \in[0,1])$. It is straightforward to see that starting from $R_{1}(1)>R_{2}(1)>0$ and some initial property rights environment, a unique equilibrium time-path $\left\{\pi^{*}(t), \rho^{*}(t)\right\}_{t=1}^{\infty}$ will be induced with the property that: $R_{1} *(t)>R_{2} *(t)>0$ for all $t$ (and hence: $R^{*}(t) \equiv\left[R_{1} *(t)+R_{2} *(t)\right]$ $>0$ and $\rho^{*}(t) \equiv R_{2} *(t) / R^{*}(t) \in(0,0.5)$ for all $\left.t\right)$. Given inherited resources $R_{1}>R_{2}>0$ (or equivalently, given: $R \equiv\left[R_{1}+R_{2}\right]>0$ and $\left.\rho \equiv R_{2} / R \in(0,0.5)\right)$ and established property rights $\pi$ in a generation, let us denote the resource inequality parameter inherited by the next generation (in the continuation equilibrium of the game) as: $r^{\dagger}(\sigma, \pi, R, \rho) \equiv F\left(\rho R, B_{2}^{\dagger}(\sigma, \pi, R, \rho), W_{2}^{\dagger}(\sigma, \pi, R, \rho)\right.$; ( $\rho$, $\pi)) /\left[F\left((1-\rho) R, B_{1}^{\dagger}(\sigma, \pi, R, \rho), W_{1}^{\dagger}(\sigma, \pi, R, \rho) ;(\rho, \pi)\right)+F\left(\rho R, B_{2}^{\dagger}(\sigma, \pi, R, \rho), W_{2}^{\dagger}(\sigma, \pi, R, \rho) ;(\rho\right.\right.$, $\pi))$ ]. Consider the case when: $\{y(\rho, \pi) / z(\rho, \pi)>(2-\sigma) / \sigma\}$ for all $\rho$ and $\pi$. We cannot directly invoke Propositions 10 and 11 here since the $R L M$ is not just a function of $R_{i}, B_{i}$ and $W_{i}$. In any generation in which established rights $\pi<1$ and $\rho \in(\tilde{\rho}(\pi \mid \sigma), 0.5)$, since $\{y(\rho, \pi) / z(\rho, \pi)>(2-$ $\sigma) / \sigma\}$ so, as we established in the proof of proposition 11 , we will get: $r^{\dagger}(\sigma, \pi, R, \rho)<\rho$. In any generation in which established rights $\pi<1$ and $\rho \leq \tilde{\rho}(\pi \mid \sigma)$, since $\{y(\rho, \pi) / z(\rho, \pi)>(2-\sigma) / \sigma\}$ so, as we established in the proof of proposition 11, we will get: $r^{\dagger}(\sigma, \pi, R, \rho)<\tilde{\rho}(\pi \mid \sigma)$. So, if the equilibrium path is such that $\rho^{*}(t) \leq 0.5 \sigma /(1+\sigma)$ in some generation $t$, then resource inequality will remain there for all later generations. We will next establish (like we did in the proof of Proposition 10) that the equilibrium path, starting from $\rho(1) \in\left(0.5 \sigma /(1+\sigma), \rho^{+}(\sigma)\right)$, does not converge weakly above $0.5 \sigma /(1+\sigma)$. We will achieve this by assuming the contrary hypothesis and then establishing a contradiction: Suppose that $\left\{\pi^{*}(t), \rho^{*}(t)\right\}_{t=1}^{\infty}$ converges to $\rho^{\prime} \in$ $[0.5 \sigma /(1+\sigma), \rho(1))$; then, $\rho^{*}(t)$ will come arbitrarily close to $\rho^{\prime}$ for large $t$ (since resource inequality will keep strictly increasing over time), and eventually (i.e., for all large $t): \pi^{*}(t)=$ $\pi^{\dagger}\left(\rho^{*}(t) \mid \sigma\right)$. Define the following function of $\rho \in\left[0.5 \sigma /(1+\sigma), \rho^{+}(\sigma)\right)$ given $\sigma \in[0.5,1)$ and $R>$ 0: $J(\rho \mid \sigma) \equiv r^{\dagger}\left(\sigma, \pi^{\dagger}(\rho \mid \sigma), R, \rho\right)<\rho$. Note that all the $R$ 's will get cancelled and so $J(\rho \mid \sigma)$ is independent of $R$. It is easily shown (as in the proof of Proposition 10) that $J(\rho \mid \sigma)$ is continuous over its domain. Then, using the fact that for all large $t$ we have $\pi^{*}(t)=\pi^{\dagger}\left(\rho^{*}(t) \mid \sigma\right)$, we get that for all large $t$ : $\rho^{*}(t+1)=J\left(\rho^{*}(t) \mid \sigma\right)$. Using this fact and the fact that $\rho^{*}(t)$ converges to $\rho^{\prime}$, we can show that the function $J(\rho \mid \sigma)$ is discontinuous at $\rho=\rho^{\prime}$, which is a contradiction to the fact that the function is continuous over its domain. Hence, our assumption that $\left\{\pi^{*}(t), \rho^{*}(t)\right\}_{t=1}^{\infty}$ converges to $\rho^{\prime} \geq 0.5 \sigma /(1+\sigma)$ was incorrect, and so for all large enough $t$ we will get: $\rho^{*}(t) \leq 0.5 \sigma /(1+\sigma)$. Since $0.5 \sigma /(1+\sigma)<\rho^{-}(\sigma)$, so using Proposition 8 we get that the equilibrium path $\left\{\pi^{*}(t), \rho^{*}(t)\right\}_{t=1}^{\infty}$ devolves to anarchy for all $\rho(1) \in\left(0, \rho^{+}(\sigma)\right)$. (It is straightforward to check the proof of Proposition 8 to see that we do not require the $R L M$ to be 'well-behaved' or for it to only depend on $R_{i}, B_{i}$ and $W_{i}$ for that result to hold. Having already established that $R_{1} *(t)>R_{2} *(t)>0$ for all $t$, we can directly invoke Proposition 8 here.) The case when $\{y(\rho, \pi) / z(\rho, \pi)<1 / \sigma\}$ for all $\rho$ and $\pi$ is 
handled using similar arguments and it can be shown that the equilibrium path $\left\{\pi^{*}(t), \rho^{*}(t)\right\}_{t=1}^{\infty}$ attains perfect property rights for all $\rho(1) \in(0,0.5)$. 